\title{
Metodologia de Estimação de \\ Parâmetros de Sistemas Dinâmicos \\ Não-Lineares com Aplicação em \\ Geradores Síncronos
}

Elmer Pablo Tito Cari

Tese de Doutorado apresentada à Escola de Engenharia de São Carlos da Universidade de São Paulo, como parte dos requisitos para obtenção do Título de Doutor em Engenharia Elétrica.

ORIENTADOR: Prof. Dr. Luís Fernando Costa Alberto CO-ORIENTADOR: Prof. Dr. Newton Geraldo Bretas

São Carlos 
AUTORIZO A REPRODUÇÃO E DIVULGAÇÃO TOTAL OU PARCIAL DESTE TRABALHO, POR QUALQUER MEIO CONVENCIONAL OU ELETRÔNICO, PARA FINS DE ESTUDO E PESQUISA, DESDE QUE CITADA A FONTE.

Ficha catalográfica preparada pela Seção de Tratamento da Informação do Serviço de Biblioteca - EESC/USP

Tito Cari, Elmer Pablo
Metodologia de estimação de parâmetros de sistemas
dinâmicos não-lineares com aplicação em geradores
síncronos / Elmer Pablo Tito Cari ; orientador Luís
Fernando Costa Alberto, co-orientador Newton Geraldo
Bretas. -- São Carlos, 2009 .
Tese (Doutorado-Programa de Pós-Graduação em
Engenharia Elétrica e Área de Concentração em Sistemas
Elétricos de Potência -- Escola de Engenharia de São
Carlos da Universidade de São Paulo, 2009.
1. Estimação de parâmetros. 2. Gerador síncrono.
3. Sensibilidade de trajetória. 4. Sistemas não-lineares.
5. Sincronização. I. Título.




\section{FOLHA DE JULGAMENTO}

\section{Candidato: Engenheiro ELMER PABLO TITO CARI}

Tese defendida e julgada em 27/03/2009 perante a Comissão Julgadora:

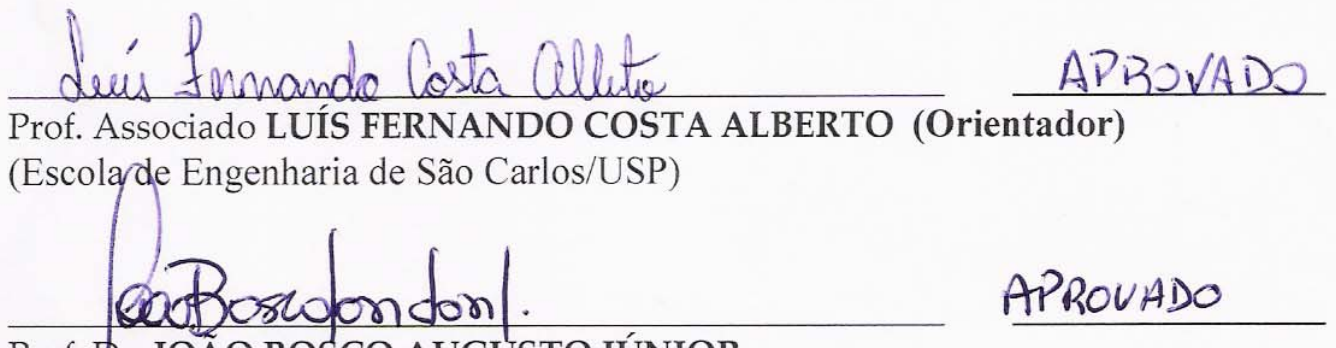

Prof. фr. JOAO BOSCO AUGUSTO JÚNIOR

(Escola de Bngenharia de São Carlos/USP)
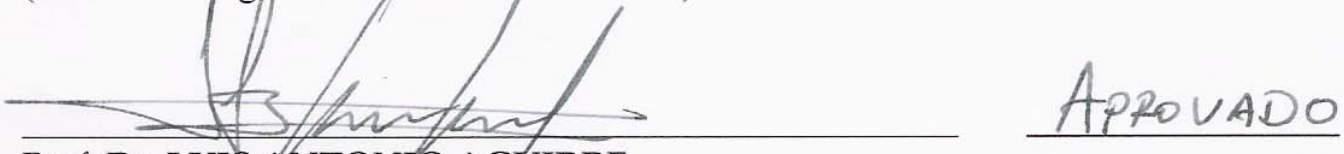

Prof. Dr. LUIS NTONIO AGUIRRE

(Universidade Federal de Minas Gerais/UFGM)
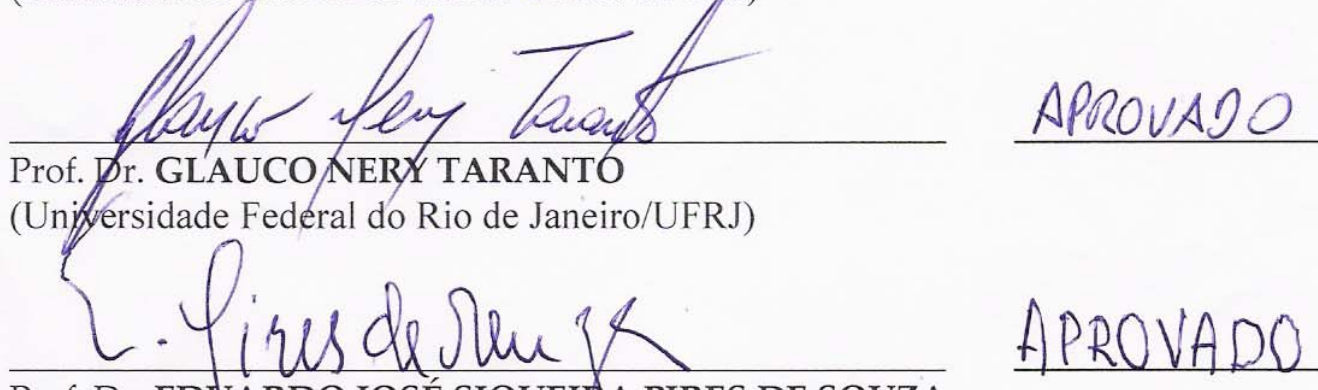

(Unizyersidade Federal do Rio de Janeiro/UFRJ)

Prof. Dr. EDUARDO JOSÉ SIQUEIRA PIRES DE SOUZA

(Pontifícia Universidade Católica/PUe/RJ)

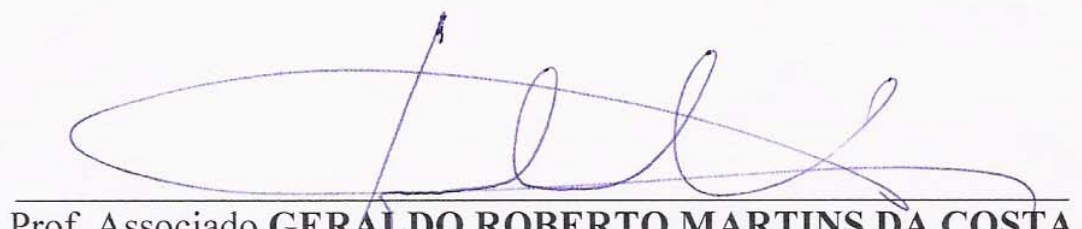

Prof. Associado GERALDO ROBERTO MARTINS DA COSTA

Coordenador do Programa de Pós-Graduação em Engenharia Elétrica e Presidente da Comissão de Pós-Graduação 


\section{Dedicatória}

Com muito amor dedico este trabalho: aos meus pais Julian (em memória) e Juana, aos meus irmãos e irmãs Hugo, Miguel e Cesar, Beatriz, Angélica, Leonarda e Olga, as minhas sobrinhas e sobrinhos Cyntia, Ana, Sofia e Anthony, Jordan, Diana, aos meus cunhados e cunhadas Marcelino, Raul, Edwin e Haidee, e a todos os meus familiares. 


\section{Agradecimentos}

Ao nosso amado Senhor Jesus por ter me mostrado as riquezas insondáveis de Cristo.

- Ao Professor Luís Fernando Costa Alberto pela sua excelente orientação durante o presente trabalho.

- Ao Professor Newton Geraldo Bretas pela sua co-orientação e amizade.

- Aos Professores João Bosco e Rodrigo pela amizade compartilhada no LACOSEP.

- Aos companheiros do laboratório LACOSEP, aqueles que encontrei ao chegar, aqueles que já foram e ainda aqueles que estão chegando.

- Aos irmãos da igreja em São Carlos, pelas suas orações.

- Aos irmãos da minha república: Fabiano, Daniel, Douglas, Paulo, Jorge e Julio, pela amizade. 


\section{Resumo}

Cari, Elmer Pablo Tito,(2009). Metodologia de Estimação de Parâmetros de Sistemas Dinâmicos Não-Lineares com Aplicação em Geradores Síncronos. Tese (Doutorado) - Escola de Engenharia de São Carlos, Universidade de São Paulo, São Carlos, 2009.

Este trabalho apresenta uma nova metodologia para estimar parâmetros de geradores síncronos baseada na análise de sensibilidade de trajetória. Esta nova metodologia foi concebida com o objetivo de suplantar dificuldades de convergência que a metodologia de sensibilidade de trajetória tradicional apresenta devido a: (i) baixa robustez com relação aos valores iniciais dos parâmetros e ruído nas medidas, (ii) impossibilidade de lidar com singularidades que podem se apresentar nas equações algébricas do modelo de EAD (equações algébrica-diferenciais) que levam a inexistência de soluções, especialmente quando os parâmetros estão distantes dos valores verdadeiros. Apesar de ter sido desenvolvida para resolver o problema de estimação de parâmetros do gerador síncrono, a metodologia é geral e pode ser aplicada para uma classe grande de sistemas dinâmicos não-lineares. Neste sentido, a principal contribuição desta tese é a proposição de uma nova metodologia baseada na sensibilidade de trajetória para estimar parâmetros de sistemas dinâmicos não-lineares restritos, ou seja, modelados por EADs. Mais precisamente, relaxa-se a restrição de igualdade do sistema dinâmico, substituindo-a por uma formulação alternativa baseada na minimização da função algébrica do modelo de EAD. Uma segunda contribuição desta tese está relacionada à modelagem do gerador. Neste sentido, a escolha de variáveis de estado, das entradas e saídas, é fundamental para o sucesso da metodologia de estimação 
de parâmetros. Nesta tese, estas escolhas permitem que os parâmetros mecânicos e elétricos possam ser estimados independentemente. Para estimar os parâmetros elétricos, o gerador é modelado por um conjunto de EADs para que os seguintes requisitos práticos sejam atendidos: (i) estimar os parâmetros a partir de medidas de perturbações obtidas com o gerador em operação, (ii) usar apenas medidas de fácil acesso, (iii) não depender dos parâmetros da rede. Como resultado final, propõe-se um algoritmo que combina a nova metodologia de sensibilidade de trajetória para sistemas restritos com um algoritmo de estimação em duas fases para estimar os parâmetros do gerador síncrono. A metodologia proposta é robusta aos valores iniciais dos parâmetros e atende aos requisitos práticos mencionados anteriormente. Além disso, a estimação do ângulo de potência é um subproduto da metodologia proposta.

Palavras-Chave: Estimação de parâmetros. Gerador síncrono. Sensibilidade de trajetória. Sistemas não-lineares. Sincronização. 


\section{Abstract}

Cari, Elmer Pablo Tito (2009). Parameter Estimation Methodology of Dynamic Nonlinear Systems with Application in Synchronous Generators. Tese (Doctorate study)-Escola de Engenharia de São Carlos, Universidade de São Paulo, São Carlos, 2009.

This research proposes a new methodology to estimate parameters of synchronous generators based on trajectory sensitivity analysis. This new methodology was created to overcome convergence difficulties presented by the traditional trajectory sensitivity methodology due to: (i) Low robustness with relation to initial parameter values and noisy measurements; and (ii) singularities in the algebraic equation of the model of differential-algebraic equations (DAEs) that lead to the nonexistence of solutions, especially when the parameters are far from of the true values. Although the methodology has been developed to solve the synchronous generator problem, it is general and can be used for many types of nonlinear dynamic systems. Therefore, the main contribution of this thesis is the proposal of a new methodology based on trajectory sensitivity to estimate parameters of nonlinear dynamic systems with constraints, i.e., systems modeled by DAEs. More precisely, the equality constraint of the dynamic system is relaxed by an alternative formulation based on the minimization of the algebraic function of the model of DAEs. A second contribution of the thesis is related to the model of the generator. For this intention, the selection of the state variables, inputs and outputs is fundamental for the success of the parameter estimation methodology. In this thesis, this selection allows the generator's mechanical and electrical parameters be estimated independently. In order to estimate the electrical pa- 
rameters, the generator is modeled by a set of convenient DAEs to fulfill the following practical requirements: (i) estimation of the generator parameters from the disturbance measurements obtained with the machine in operation; (ii) use of easily accessible measurements; and (iii) independence of the network parameter. As a final result, an algorithm which combines the new methodology of trajectory sensitivity to constrained systems with the two-stage estimation algorithm is proposed to estimate the generator parameters. This proposed methodology is robust for parameter's initial values and fulfills the practical requirements above mentioned. In addition, the estimation of the power angle is a byproduct of the proposed methodology.

Key-words: Parameter estimation. Synchronous generator. Trajectory sensitivity. Nonlinear systems. Synchronization. 


\section{Lista de Figuras}

2.1 Etapas do processo de identificação. . . . . . . . . . . . . . . . 10

2.2 Etapas do processo de estimação de parâmetros. Os parâmetros são ajustados a fim de minimizar a função objetivo. . . . . . . . . 11

2.3 Região de estimação do algoritmo de ajuste. . . . . . . . . . . . 15

2.4 Saída e funções de sensibilidade para o Exemplo 2.2 . . . . . . . . 17

3.1 Continuidade com relação aos parâmetros. . . . . . . . . . . . . 33

3.2 Estimação de parâmetros. (a) com a abordagem original, (b) com o acoplamento mestre-escravo. . . . . . . . . . . . . . . 34

3.3 Gráfico da saída e funções de sensibilidade para o Exemplo 3.1 . . 36

4.1 Metodologia de sensibilidade de trajetória na formulação tradicional. 38

4.2 Metodologia de sensibilidade de trajetória usando o acoplamento mestre-escravo. . . . . . . . . . . . . . . . . . . . . . . . . 38

4.3 Estimação de parâmetros do sistema de Lorenz usando a metodologia de sensibilidade de trajetória tradicional. . . . . . . . . . . 44

4.4 Saídas do sistema real e auxiliar considerando que os parâmetros do sistema auxiliar estão $+2 \%$ distantes dos valores corretos. . . . 44

4.5 Funções de sensibilidade de trajetória da variável $z_{1}$ em relação aos parâmetros. . . . . . . . . . . . . . . . . . . 45

4.6 Estimação de parâmetros do sistema de Lorenz baseada em sensibilidade de trajetória e acoplamento mestre-escravo. . . . . . . . . 47

4.7 Saída \#1 antes e após a convergência dos parâmetros. Todas as medidas possuem ruído gaussiano de média zero e desvio padrão 5\%. 49 
4.8 Convergência dos parâmetros ao longo das iterações. . . . . . . . . 50

4.9 Estimação de parâmetros do sistema de Lorenz com a metodologia de sensibilidade de trajetória mudando as entradas e saídas. . . . 51

4.10 Estimação de parâmetros do sistema de Chua baseada em sensibilidade de trajetória incluindo o acoplamento mestre-escravo. . . . 53

5.1 Dificuldades e propostas de solução para a estimação dos parâmetros da máquina síncrona . . . . . . . . . . . . . . . . 60

5.2 Estratégia para estimação de parâmetros. As medidas são obtidas durante uma perturbação no SEP e utilizadas pela metodologia de estimação de parâmetros. . . . . . . . . . . . . . . . . . . . . . 61

5.3 Condições de Operação no processo de estimação. . . . . . . . . . 62

5.4 Sistema de potência usado para os testes. As medidas são coletadas durante uma perturbação no SEP. . . . . . . . . . . . . . . . .

5.5 Sistemas de referência, da máquina síncrona $(d-q)$ e da rede externa $\left(I_{m}-R_{e}\right) \ldots \ldots \ldots \ldots$. . . . . . . . . 6 64

5.6 Diagrama de blocos para a estimação dos parâmetros mecânicos. . 67

5.7 Convergência dos parâmetros mecânicos ao longo das iterações. . . 69

5.8 Diferença entre as saídas do sistema real e sistema auxiliar. . . . . 69

5.9 Estimação de parâmetros elétricos utilizando o modelo original do gerador síncrono. . . . . . . . . . . . . . 70

5.10 Estimação de parâmetros elétricos usando o modelo tradicional do gerador síncrono com acoplamento mestre-escravo. . . . . . . . . . 72

5.11 Sistema de referência da máquina síncrona (d-q), do sistema de potência $\left(I_{m}-R_{e}\right)$ e a proposta $(u-v$, na tensão terminal $)$. . . . . 75

5.12 Diagrama de blocos para a estimação dos parâmetros com o modelo do gerador modificado. . . . . . . . . . . . . 76

5.13 Estimação de parâmetros elétricos com a metodologia de sensibilidade de trajetória na formulação tradicional. . . . . . . . . . . . 81

5.14 Estimação de parâmetros com a metodologia de sensibilidade de trajetória com a abordagem de minimização. . . . . . . . . . . . . 85 
5.15 Comparação da região de convergência para a metodologia de sensibilidade de trajetória tradicional e com a abordagem de minimização para os parâmetros $x_{d}^{\prime}$ e $x_{q}^{\prime}$. . . . . . . . . . . . . 86

5.16 Matriz $\Gamma$ obtida a partir das equações da máquina síncrona. $\mathrm{Na}$ parte superior é possível observar a ordem original dos parâmetros. 87

5.17 Função $g(.)^{2}$ no começo do processo de estimação. . . . . . . . . . 89

5.18 Função $g(.)^{2}$ no final do processo de estimação. . . . . . . . . . . 89

5.19 Evolução de $J(p)$ ao longo das iterações. . . . . . . . . . . . . . . 90

$5.20 \Delta \beta(t)$ após a convergência dos parâmetros. . . . . . . . . . . . . . 90

5.21 Potência ativa no início do processo de estimação com ruído nas medidas. . . . . . . . . . . . . . . . . . . . . . 91

5.22 Potência ativa após a convergência dos parâmetros com ruído nas medidas. . . . . . . . . . . . . . . . . . . . . . . 91

5.23 Potência reativa no início do processo de estimação com ruído nas medidas. . . . . . . . . . . . . . . . . . . . . 92

5.24 Potência reativa após a convergência dos parâmetros com ruído nas medidas. . . . . . . . . . . . . . . . . . . . . . . . 92

$5.25 \Delta \beta(t)$ entre os sistemas real e auxiliar no começo do processo de estimação. . . . . . . . . . . . . . . . . . . . . . . 92

$5.26 \Delta \beta(t)$ entre os sistemas real e auxiliar no final do processo de estimação . . . . . . . . . . . . . . . . . . . . . . 92 


\section{Lista de Tabelas}

4.1 Resultados com a metodologia de sensibilidade de trajetória (formulação tradicional). . . . . . . . . . . . . . . . . . . 39

4.2 Resultados com a metodologia de sensibilidade de trajetória com acoplamento mestre-escravo. . . . . . . . . . . . . . . . . . . . 40

4.3 Estimação dos parâmetros usando o acoplamento mestre-escravo. 48

4.4 Estimação dos parâmetros do sistema de Lorenz baseada em sensibilidade de trajetória incluindo o acoplamento mestre-escravo e com ruído nas medidas. . . . . . . . . . . . . . . . . . . . . . . . . 49

4.5 Estimação dos parâmetros do sistema de Lorenz com uma disposição diferente das entradas e saídas. . . . . . . . . . . . . . 51

4.6 Estimação dos parâmetros do sistema de Chua utilizando sensibilidade de trajetória incluindo o acoplamento mestre-escravo. . . . 53

5.1 Estimação dos parâmetros mecânicos. . . . . . . . . . . . . . . . . 68

5.2 Estimação dos parâmetros mecânicos com ruído nas medidas. . . . 68

5.3 Estimação dos parâmetros elétricos do gerador utilizando o modelagem original. . . . . . . . . . . . . . . . . . . . . 71

5.4 Estimação de parâmetros da máquina síncrona para a opção 2. . 73

5.5 Estimação de parâmetros da máquina síncrona para a opção 3. . . 77

5.6 Classificação dos parâmetros segunda sua influência na saída. . . . 88

5.7 Estimação em duas fases utilizando o algoritmo de sensibilidade de trajetória com abordagem de minimização. . . . . . . . . . . . 89 
5.8 Influência do ruído nas medidas na metodologia de sensibilidade de trajetória com abordagem de minimização e o algoritmo de estimação em duas fases. . . . . . . . . . . . . . . . . . . . . . . . 91

5.9 Comparação das principais alternativas para estimar os parâmetros elétricos do gerador. . . . . . . . . . . . . . . . . . . . 93 


\section{Conteúdo}

Dedicatória iii

$\begin{array}{ll}\text { Agradecimentos } & \mathrm{v}\end{array}$

Resumo vii

$\begin{array}{lll}\text { Abstract ix } & \text { ix }\end{array}$

Lista de Figuras

Lista de Tabelas $\quad$ xiii

1 Introdução 1

1.1 Apresentação . . . . . . . . . . . . . . . . . . . . . . . . . . 1

1.2 Organização da Tese . . . . . . . . . . . . . . . . . . . . 4

$\begin{array}{lll}2 & \text { Metodologia de Estimação de Parâmetros } & 7\end{array}$

2.1 Introdução . . . . . . . . . . . . . . . . . . . . . . . . 7

2.2 Processo de Identificação . . . . . . . . . . . . . . . . . . . . . . 8

2.2.1 Obtenção dos dados ou medidas . . . . . . . . . . . . . . . 8

$2.2 .2 \quad$ Escolha da estrutura do modelo . . . . . . . . . . . . . . . 9

2.2.3 Técnica de estimação ou critério de ajuste de parâmetros . 9

2.3 Processo de Estimação de Parâmetros . . . . . . . . . . . . . . . . 10

2.4 Otimização Não-linear . . . . . . . . . . . . . . . . . . . . . . . 11

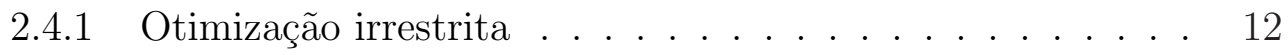

2.4 .2 Otimização com restrições de igualdade . . . . . . . . . . . 12 
2.4 .3 Otimização com restrições de desigualdade . . . . . . . . . 13

$2.4 .4 \quad$ Estimativa de parâmetros . . . . . . . . . . . . . . . 14

2.5 Funções de Sensibilidade de Trajetória . . . . . . . . . . . . . . . 14

2.5.1 Funções de sensibilidade de trajetória de sistemas lineares invariantes no tempo (LIT) . . . . . . . . . . 18

2.5.2 Funções de sensibilidade de trajetória de sistemas dinâmicos não-lineares . . . . . . . . . . . . . . . . . . 19

2.5.3 Funções de sensibilidade de trajetória para sistemas algébricodiferenciais: Abordagem de minimização . . . . . . . . . . 22

2.6 Metodologia de estimação de parâmetros baseado em sensibilidade de trajetória . . . . . . . . . . . . . . . 25

2.6.1 Algoritmo de estimação de parâmetros com a formulação tradicional . . . . . . . . . . . . . . . . 26

2.6.2 Algoritmo de estimação de parâmetros com a abordagem de minimização . . . . . . . . . . . . . . . . 27

2.7 Estimação de Parâmetros em Duas Fases . . . . . . . . . . . . . . 28

2.7.1 Classificação dos parâmetros segundo sua influência na saída do modelo . . . . . . . . . . . . . . . . . . . 29

2.7.2 Algoritmo de estimação de parâmetros em duas fases . . . 29

$\begin{array}{lll}3 & \text { Sincronização Mestre-Escravo } & 31\end{array}$

3.1 Continuidade de Soluções de EDOs . . . . . . . . . . . . . . . . 32

3.2 Acoplamento Mestre-Escravo . . . . . . . . . . . . . . . . . . . . 33

4 Estimação de Parâmetros de Sistemas Dinâmicos Não-Lineares 37

4.1 Estimação de Parâmetros do Sistema Massa-Mola . . . . . . . . . 37

4.2 Estimação de Parâmetros do Sistema de Lorenz . . . . . . . . . . 40

4.2.1 Metodologia de sensibilidade de trajetória na formulação tradicional . . . . . . . . . . . . . . . . 42

4.2.2 Metodologia de sensibilidade de trajetória com acoplamento mestre-escravo. . . . . . . . . . . . . . . . . 45 
4.2 .3 Mudando as entradas e as saídas . . . . . . . . . . . . . . 49

4.3 Estimação de Parâmetros do Sistema de Chua . . . . . . . . . . . 51

5 Estimação de Parâmetros do Gerador Síncrono 55

5.1 Introdução . . . . . . . . . . . . . . . . . . 55

5.2 Dificuldades e alternativas de solução . . . . . . . . . . . . 56

5.3 Esquema Para Estimar os Parâmetros com o Gerador em Operação 61

5.3.1 Considerações práticas para a realização dos testes . . . . 62

5.3 .2 Sistema de potência . . . . . . . . . . . . . 63

5.3 .3 Sistema de referência . . . . . . . . . . . . . 63

5.3.4 Modelo Original do Gerador Síncrono . . . . . . . . . . . 64

5.4 Estimação dos Parâmetros Mecânicos do Gerador . . . . . . . . . 66

5.4 .1 Equações de sensibilidade . . . . . . . . . . . . 67

5.4 .2 Resultados . . . . . . . . . . . . . . . . . 68

5.4 .3 Influência do ruído nas medidas . . . . . . . . . . . . . 68

5.5 Estimação dos Parâmetros Elétricos do Gerador . . . . . . . . . . 69

5.5.1 Estimação dos parâmetros elétricos utilizando o modelo original do gerador . . . . . . . . . . . . . . . . . 69

5.5.2 Estimação dos parâmetros elétricos utilizando o modelo original do gerador e com acoplamento mestre-escravo . . . . 71

5.5.3 Estimação dos parâmetros elétricos utilizando o modelo do gerador modificado . . . . . . . . . . . . . . 74

5.5.4 Estimação dos parâmetros elétricos utilizando o modelo do gerador modificado e estimando numericamente o ângulo de potência .................... 77

$\begin{array}{lll}6 & \text { Conclusões } & 95\end{array}$

6.1 Considerações Finais e Perspectivas Futuras . . . . . . . . . . . . 99

$\begin{array}{ll}\text { A Publicações Originadas desta Pesquisa } & 109\end{array}$

A.1 Artigos Publicados em Congressos . . . . . . . . . . . . . . . . . 109

A.2 Artigos Submetidos em Revistas . . . . . . . . . . . . . . . . . 110 
A.3 Artigos Submetidos em Congressos . . . . . . . . . . . . . . . . 110

B Equações do Gerador com a Abordagem de Minimização. 111

B.1 Sistema Auxiliar . . . . . . . . . . . . . . . . . . . . . . . . 111

B.2 Equações de Sensibilidade e Funções de Sensibilidade de Trajetória 115 


\section{Capítulo 1}

\section{Introdução}

\subsection{Apresentação}

Este trabalho foi desenvolvido considerando a necessidade das empresas do setor elétrico, principalmente na área de sistemas de potência, de conhecer com precisão os parâmetros dos modelos da máquina síncrona e controladores associados (sistemas de excitação, regulador de velocidade, etc.) usados em simulações computacionais. O uso de modelos representativos e a escolha correta de parâmetros é de vital importância na medida em que diversas ações de operação e controle são obtidas a partir de diversos estudos, tais como: estudos de estabilidade, proteção, planejamento e expansão do SEP (Sistema Elétrico de Potência) entre outros. Entretanto, grandes dificuldades são encontradas quando as simulações não reproduzem com precisão o comportamento dinâmico real do SEP. Condições operativas inseguras, imprevistas e decisões incorretas podem levar o sistema ao colapso. O motivo principal destas incoerências deve-se ao fato de o banco de dados, utilizado nestas simulações, possuirem incertezas. Alguns dos motivos destas incertezas são: perda dos dados originais, ajustes de ganhos não registrados, mudança dos parâmetros ${ }^{1}$ devido ao envelhecimento do equipamento, etc.. No caso do gerador síncrono, além dos motivos mencionados anteriormente, os parâmetros fornecidos pelo fabricante (obtidos de testes off-line) também podem

\footnotetext{
${ }^{1}$ Os parâmetros da máquina síncrona são, por exemplo, as reatâncias e as constantes de tempo.
} 
mudar com o gerador em operação. Isto é mais evidente tendo em mente que alguns parâmetros do gerador estão sujeitos a saturação dependendo da condição de operação.

A seguir, descrevem-se alguns casos nos quais os valores incorretos dos parâmetros utilizados em simulações trouxeram graves conseqüências para o SEP:

(a) Os eventos ocorridos no WSCC (Western System Coordinating Council) em 10 de agosto de 1996, onde as simulações indicaram operação estável do SEP enquanto que na prática, o sistema ficou instável (Dmitry et al., 1999).

(b) Oscilações prolongadas observadas na interligação Alberta e British Columbia quando as simulações prediziam o amortecimento das oscilações (Hauer et al., 2000).

(c) O corte do suprimento de energia em várias áreas do sistema elétrico sul do Peru durante os testes de estabilidade realizados pelo COES-SINAC (Comite de Operación Económica del Sistema Interconectado Nacional) em 9 de julho de 2002 em conseqüência de diversas oscilações não previstas em simulaçãd ${ }^{2}$.

Depois destes eventos algumas medidas foram adotadas. Por exemplo, o WSCC solicitou que a cada 5 anos seja feita a verificação dos modelos e parâmetros dos geradores síncronos maiores do que 10MW (Dmitry et al., 1999). Da mesma forma, o COES-SINAC pediu que se realizasse um estudo mais preciso no SEP antes da realização de testes reais.

Embora existam diversas metodologias relatadas na literatura, ainda persistem muitos problemas e inconvenientes de natureza prática para estimar parâmetros de geradores síncronos, pois os métodos muitas vezes precisam: (i) Isolar a máquina síncrona para realizar ensaios especiais; (ii) Utilizar medidas difíceis de serem obtidas tais como ângulo de rotor e fluxos magnéticos; (iii) Dispor de estimativa inicial próxima dos valores verdadeiros dos parâmetros, que, muitas vezes, é difícil de se ter.

\footnotetext{
${ }^{2}$ Fonte, página web do COES: www.coes.org.pe
} 
Entre os trabalhos relatados, foram aplicados métodos baseados em testes offline, ou seja, com a máquina desconectada do SEP. Muitos desses testes foram padronizados pelo Instituto de Engenheiros Eletricistas e Eletrônicos (IEEE, 2002). Entre eles, pode citar-se: testes de curto circuito (Soliman e Al-kandari, 1996), (Weeber, 1997), testes de rejeição de carga (Bortoni e Jardini, 2002), (Hiramatsu et al., 2006; Zali et al., 2000; Hannett e Feltes, 1993) e testes no domínio da freqüência. Entre os testes mais conhecidos no domínio da freqüência tem-se: testes de resposta em freqüência com rotor parado (Stand Still Frequency Response) (Horning et al., 1997; Dandeno et al., 1999; Chow et al., 1999), testes em circuito aberto (Open Circuit Frequency Response), entre outros. A desvantagem destes testes é que, pelas suas características, só podem ser realizados durante o período de manutenção programada da máquina síncrona.

Diversos métodos estimam os parâmetros com dados obtidos com a máquina conectada ao sistema. Eles são conhecidos como testes on-line. Muitos destes métodos utilizam modelos linearizados para a estimação. Entre os mais conhecidos, têm-se aqueles que injetam uma seqüência binária pseudo-aleatória (PRBS) (Karrari e Malik, 2004; Vermeulen et al., 2002) ou apenas um pequeno sinal no sistema de excitação a fim de obter a resposta dinâmica da máquina síncrona quando sujeita a pequenas perturbações. Existem muitas referências abordando esta técnica, ver (Karaagac e Tor, 2006; Le e Wilson, 1988; Velez e Lida, 2001; Burth et al., 1999) e (Zhengming et al., 1995), entre outros. O problema desta abordagem é encontrar um sinal adequado com suficiente informação para estimar todos os parâmetros e, além disso, que não cause instabilidade no sistema. Além disso, o teste só pode ser realizado com a aprovação prévia do operador do SEP.

Outra abordagem é utilizar os medidas de perturbações os quais podem ser disponíveis de relés digitais e oscilógrafos durante eventos próprios do SEP (desligamento de uma linha de transmissão, mudanças de carga, etc.), evitando a necessidade de realizar um teste intencional. Porém, para este objetivo é necessário trabalhar com as equações do modelo não-linear do gerador. Ao traba- 
lhar com o modelo não-liner do gerador, os problemas de divergência do processo de estimação são mais evidentes. Além disso, as condições iniciais de variáveis de estado que não são disponíveis, precisam ser consideradas como parâmetros aumentando o vetor de parâmetros a ser estimado e tornando o problema mais complicado. Com base a nossa experiência na estimação de parâmetros, os valores iniciais dos parâmetros, principalmente aqueles provenientes das condições iniciais de variáveis de estado não mensuráveis, tem uma influência fundamental no sucesso da estimação, pois pequenas alterações em relação a seus valores verdadeiros faz a metodologia divergir e impossibilita a estimação dos parâmetros. Este fato foi muito pouco relatado na literatura. Sendo assim, ainda existem muitas dificuldades e limitações de caráter prático para conseguir uma metodologia de estimação de parâmetros adequada.

Este trabalho, em continuação aos trabalhos realizados em (Cari, 2005), propõe uma metodologia de estimação de parâmetros de sistemas dinâmicos não lineares a partir de medidas do sistema real (medidas no tempo). Entre os objetivos a serem alcançados deseja-se estimar parâmetros de sistemas não-lineares e em particular do gerador síncrono conectado ao SEP (on-line) viabilizando sua aplicação em um sistema de potência real. Para isso será necessário:

a. Propor uma modelagem da máquina síncrona de tal forma que as medidas necessárias sejam de fácil obtenção.

b. Estimar os parâmetros a partir das oscilografias de perturbações (simulado computacionalmente).

c. Propor alternativas de solução aos problemas de mal-condicionamento decorrentes do processo de estimação.

\subsection{Organização da Tese}

Com a finalidade de organizar melhor esta tese, os primeiros capítulos apresentam conceitos teóricos importantes relacionados ao processo de estimação de 
parâmetros e posteriormente as aplicações da metodologia desenvolvida. Assim, o trabalho está dividido como é mostrado a seguir.

O Capítulo 2 apresenta o embasamento teórico para a estimação de parâmetros de sistemas não-lineares. Nele são mostradas as etapas e propriedades do processo de estimação e a formulação para o desenvolvimento da metodologia de estimação não-linear de parâmetros baseada na sensibilidade de trajetória. Propõe-se, nesse capítulo, uma formulação alternativa para estimar parâmetros de sistemas modelados por equações algébrico-diferenciais baseada em uma abordagem de minimização. Apresenta também um algoritmo para estimar os parâmetros em duas fases a fim de aumentar a região de convergência.

O Capítulo 3 apresenta o processo de estimação de parâmetros como um processo de sincronização e propõe-se o acoplamento "mestre-escravo" como ferramenta para alcançar sincronização e aumentar a região de convergência da metodologia de estimação.

O Capítulo 4 mostra a aplicação da metodologia desenvolvida para estimar parâmetros de sistemas dinâmicos não-lineares. Mostra-se a vantagem do acoplamento mestre-escravo no processo de estimação de parâmetros.

O Capítulo 5 mostra a aplicação da metodologia desenvolvida para estimar parâmetros de geradores síncronos. Inicialmente são analisadas as dificuldades para estimar os parâmetros do gerador em sistemas reais. Baseado nisto, propõese uma metodologia de estimação que atende diversos requisitos práticos.

O Capítulo 6 apresenta as conclusões e algumas considerações finais da presente tese.

No Apêndice A mostram-se as principais publicações originadas por esta pesquisa e no Apendice B mostram-se as equações do modelo final proposto da metodologia de sensibilidade de trajetória com abordagem de minimização para estimar parâmetros do gerador síncrono. 


\section{Capítulo 2}

\section{Metodologia de Estimação de Parâmetros}

\subsection{Introdução}

A estimação de parâmetros está situada dentro da área de identificação de sistemas. O processo de identificação de sistemas consiste em encontrar um modelo matemático que represente adequadamente saídas observadas do sistema.

O modelo matemático pode classificar-se em diferentes tipos, dependendo da finalidade de sua aplicação. Em (Aguirre, 2000; Ljung, 1997) mostram-se diversas formas de classificação do modelo matemático. O modelo matemático basicamente compõe-se de um conjunto de relações matemáticas ou equações (estrutura do modelo). As quantidades que aparecem nestas equações podem classificar-se como variáveis ou como parâmetros (constantes que acompanham as variáveis). Quando o comportamento das variáveis do sistema evolui no tempo, o sistema é dito dinâmico e utiliza-se um modelo dinâmico para sua representação. Neste caso, as equações do modelo matemático não dependem apenas de variáveis dependentes ou independentes, mas também de derivadas destas variáveis.

No exemplo a seguir, descrevem-se os elementos que compõem um modelo matemático de um sistema dinâmico contínuo.

Exemplo 2.1 Seja o sistema massa-mola modelado pela seguinte equação dife- 
rencial

$$
m \ddot{y}=u(t)-k y,
$$

Este sistema de segunda ordem pode ser decomposto em duas equações de primeira ordem, isto é, definindo $x_{1}=y$ e $x_{2}=\dot{y}$ (posição e velocidade respectivamente), obtém-se:

$$
\begin{aligned}
\dot{x}_{1} & =x_{2}, \\
\dot{x}_{2} & =-\frac{k}{m} x_{1}+\frac{u}{m}, \\
y & =x_{1}, \\
u(t) & = \begin{cases}0, & t<0 \\
u_{o}, & t \geq 0 .\end{cases}
\end{aligned}
$$

sendo $(k, m)$ parâmetros, u a entrada (força externa) $e\left(x_{1}, x_{2}\right)$ as variáveis de estado do sistema (que podem formar parte do conjunto de medidas). Além disso precisa-se de duas condições iniciais $x_{1 o}, x_{2 o}$, as quais também podem formar parte do conjunto de parâmetros.

\subsection{Processo de Identificação}

O processo de identificação de sistemas é constituído de três etapas básicas: (i) obtenção dos dados ou medidas, (ii) escolha da estrutura do modelo e (iii) estimação de parâmetros.

\subsubsection{Obtenção dos dados ou medidas}

A primeira etapa do processo de identificação é a obtenção dos dados ou medidas. No presente estudo, interessa-se em modelos dinâmicos, portanto é necessário a realização de ensaios ou testes a fim de se obter informações da resposta dinâmica do sistema. Estas informações são obtidas de grandezas disponíveis ou possíveis de serem medidas. As medidas devem ter informação suficiente da resposta dinâmica do sistema durante o intervalo medido para evitar problemas de 
mal-condicionamento pela pouca informação. Além disso, dependendo do teste realizado alguns parâmetros podem ser estimáveis e outros não. Como ilustração, no sistema massa-mola do Exemplo 2.1, utilizando-se do mesmo conjunto de medidas, os parâmetros podem ser ou não estimáveis dependendo do teste realizado. Quando a entrada $u$ é igual a zero, o sistema é excitado com condições iniciais diferentes de zero (velocidade e posição), as medidas não fornecem informações suficientes para estimar os parâmetros $k$ e $m$ simultaneamente, porém, aplicandose um degrau na entrada $u$, pode-se estimar os dois parâmetros simultaneamente (ver (Cari, 2005)).

\subsubsection{Escolha da estrutura do modelo}

Nesta etapa do processo de identificação, definem-se as equações (algébricas e/ou diferenciais) do sistema estudado. Esta representação pode variar de expressões simples a expressões detalhadas segundo a aplicação e a finalidade. Por exemplo, em SEP, quando precisa-se estudar o comportamento estático da rede, o gerador síncrono é usualmente representado pelo modelo de regime permanente que consiste em uma fonte de tensão fixa em série com uma reatância de eixo direto. Em estudos de proteção e estabilidade, onde precisa-se conhecer o comportamento dinâmico do sistema, o gerador síncrono deve ser representado pelo modelo clássico, transitório ou sub-transitório (dependendo da precisão dos resultados) os quais possuem equações diferenciais no modelo (Kundur, 1994).

Neste trabalho, admite-se que a estrutura do modelo matemático seja conhecida (obtida de modelos padronizados). Por exemplo, para estudos de estabilidade em SEP dispõe-se de modelos padronizados da máquina síncrona (IEEE, 2002).

\subsubsection{Técnica de estimação ou critério de ajuste de parâ- metros}

Nesta etapa, com os dados coletados e com a estrutura do modelo conhecida, efetua-se o processo de estimação de parâmetros o qual consiste em encontrar um conjunto de parâmetros que melhor represente o sistema real. Para isto, 
utiliza-se um algoritmo de ajuste de parâmetros.

Na Figura 2.1 mostra-se um resumo destas etapas. Uma vez identificado o modelo, a parte da validação é feita verificando se a resposta do modelo reproduz adequadamente os dados medidos.

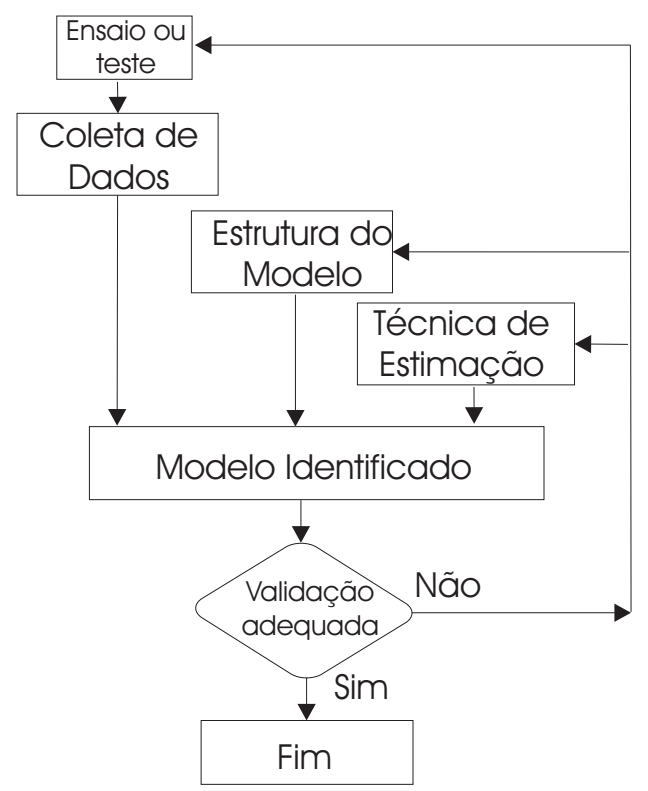

Figura 2.1: Etapas do processo de identificação.

A maior parte deste trabalho tem como foco a terceira parte do processo de identificação no qual serão desenvolvidos dois algoritmos de estimação de parâmetros. Algumas considerações importantes também serão estudadas sobre a obtenção das medidas e da estrutura do modelo.

\subsection{Processo de Estimação de Parâmetros}

Como foi visto anteriormente, o processo de estimação de parâmetros está localizado na terceira etapa do processo de identificação. Nesta fase, utiliza-se um algoritmo ou técnica de ajuste de parâmetros que aproxime o comportamento do modelo ao comportamento do Sistema real. Em geral este processo pode ser dividido em duas fases:

a) Definição da função objetivo $J(p)$ : A função objetivo $J(p)$ mede a proximidade entre os dados medidos (sistema real) e os dados obtidos a partir do modelo 
(sistema auxiliar). Usualmente esta função está baseada em alguma norma de um vetor de erro.

b) Procura de $p^{*}$ que minimiza $J(p)$ : Por meio de um procedimento de ajuste de parâmetros (que pode ser iterativo) busca-se o vetor de parâmetros que minimiza a função objetivo $J(p)$.

Neste trabalho, utiliza-se como função objetivo o quadrado das diferenças das saídas, a qual está baseada na norma 2 no espaço das funções contínuas definidas em certo intervalo da reta (método de mínimos quadrados) e a minimização será realizada por meio do método de Newton . Na Figura 2.2, mostram-se as fases do processo de estimação. Uma vez definida a estrutura do modelo do sistema, e com as medidas coletadas, o algoritmo de ajuste de parâmetros minimiza a função objetivo por intermédio de um processo de otimização.

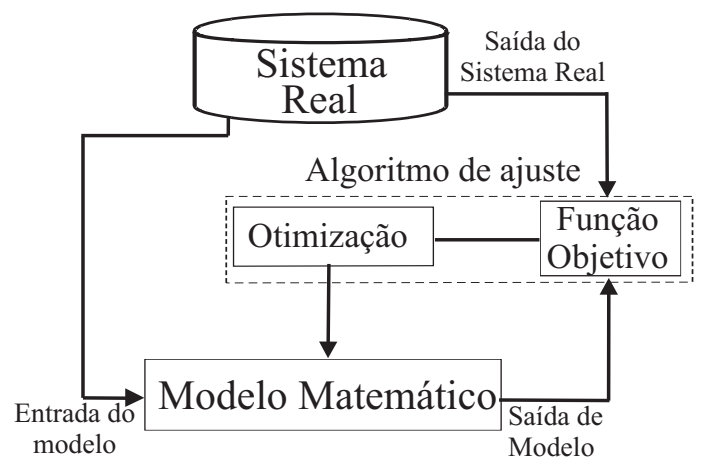

Figura 2.2: Etapas do processo de estimação de parâmetros. Os parâmetros são ajustados a fim de minimizar a função objetivo.

\subsection{Otimização Não-linear}

O processo de estimação é formulado como um problema de otimização no qual a função objetivo deve ser minimizada. Na seqüência, um resumo da teoria de programação não-linear será apresentado.

Quando os parâmetros podem assumir qualquer valor, o processo é denominado de otimização irrestrita. Se a estimação dos parâmetros estiver condicionada a certas restrições, o processo é denominado otimização restrita. 
Existem dois tipos de restrições: restrições de igualdade e restrições de desigualdade. A região, no espaço de parâmetros, onde os parâmetros satisfazem as restrições, denomina-se região factível (Figura 2.3). Por exemplo, a estimação dos parâmetros da máquina síncrona está restrita ao fato de que os parâmetros devem ser positivos e alguns maiores do que outros. Portanto a região factível limita-se ao conjunto de valores de parâmetros que satisfazem tais restrições.

\subsubsection{Otimização irrestrita}

Na seqüência serão apresentadas algumas condições de otimalidade (para o caso de minimização de uma função) para problemas sem restrição. Para isto, apresentam-se algumas definições.

Definição 2.1 Uma função $f: R^{n} \rightarrow R$ é dita de classe $C^{n}$ se for contínua e suas " $n$ " primeiras derivadas parciais existirem e forem contínuas.

Definição 2.2 Dada a função $f: R^{n} \rightarrow R$. Um ponto $x^{*} \in R^{n}$, é chamado mínimo local se existir um escalar $\delta>0$ tal que $f\left(x^{*}\right) \leq f(x)$ para qualquer $x$ tal que $\left\|x-x^{*}\right\|<\delta$. Se $f\left(x^{*}\right) \leq f(x)$ para qualquer $x \in R^{n}$, então $x^{*}$ é chamado de minimo global.

\section{Condições de suficiência para obtenção do ótimo irrestrito}

Seja $f(x)$ uma função de classe $C^{2}$, as condições suficientes para que $x^{*} \in R^{n}$ seja mínimo local de $f(x)$ são as seguintes:

1) $\nabla f\left(x^{*}\right)=0$ (gradiente no ponto $x^{*}$ igual a zero).

2) $d^{T} H\left(x^{*}\right) d>0, \forall d \in R^{n} \mid d \neq 0$ (Hessiana definida positiva no ponto $x^{*}$ ).

\subsubsection{Otimização com restrições de igualdade}

O problema de otimização com restrição de igualdade do tipo $h(x)=0$ pode ser transformado em um problema sem restrição utilizando-se o seguinte teorema.

Teorema 2.1 (Bazaraa, 1979) Seja $x^{*}$ um ponto que atende $h(x)=0$ (dentro da região factivel) com $h: R^{n} \rightarrow R^{m}$. Além disso, se $x^{*}$ for mínimo local de $f(x)$ 
sujeito à restrição $h(x)=0$, então existe um vetor $\lambda^{T} \in R^{m}$ tal que $\nabla f\left(x^{*}\right)+$ $\lambda^{T} \nabla h\left(x^{*}\right)=0$. Onde $\lambda$ é o vetor dos multiplicadores de Lagrange.

Em outras palavras, transforma-se o problema original restrito

$\min f(x)$

s.a $h(x)=0$,

no seguinte problema irrestrito

$\min L(x, \lambda)=f(x)+\lambda^{T} h(x)$ (Função Lagrangiana adjunta).

\section{Condições necessárias de $1^{\circ}$ Ordem}

As seguintes condições são necessárias para que $x^{*}$ seja mínimo local da função $f(x)$ de classe $C^{2}$ :

$\nabla_{x} L\left(x^{*}, \lambda\right)=0$, ou em forma equivalente $\nabla f(x)+\sum_{i=1}^{m} \lambda_{i} \nabla h_{i}(x)=0$, $\nabla_{\lambda} L\left(x^{*}, \lambda\right)=0$, ou em forma equivalente $h_{i}\left(x^{*}\right)=0, \quad i=1, \ldots, m$.

\subsubsection{Otimização com restrições de desigualdade}

As condições necessárias para o ótimo com restrições de desigualdade são conhecidas também como condições de KKT (Karush-Kuhn-Tucker).

Seja o problema

$$
\begin{aligned}
& \min f(x) \\
& \text { s.a } g(x) \leq 0,
\end{aligned}
$$

em que $g(x)=\left(g_{1}(x), \ldots, g_{m}(x)\right) ; x \in R^{n} ; f$ e $g$ são de classe $C^{1}$. Uma condição necessária para que $x^{*}$ seja mínimo local do problema com restrições, é que $\exists \lambda^{* T}=\left[\lambda_{1}^{*}, \lambda_{2}^{*}, \ldots, \lambda_{m}^{*}\right]$, tal que

$\lambda_{i}^{*} \geq 0, \quad i=1, \ldots, m$,

$g\left(x^{*}\right) \leq 0$,

$\lambda^{* T} g\left(x^{*}\right)=0$,

$\nabla f\left(x^{*}\right)+\lambda^{* T} \nabla g\left(x^{*}\right)=0$.

Mais detalhes a respeito das condições necessárias e suficientes para otimizar uma função sob restrições são encontradas em (Bazaraa, 1979). 


\subsubsection{Estimativa de parâmetros}

As estimativas dos parâmetros são obtidas por intermédio de um processo iterativo. Partindo de um conjunto inicial de parâmetros $\left(p_{1}\right)$, o algoritmo de ajuste gera uma seqüência de parâmetros $p_{2}, \ldots, p_{i}, p_{i+1}, \ldots$ O procedimento termina com a convergência da seqüência gerada para um conjunto de parâmetros próximo do vetor de parâmetros verdadeiro, $p^{*}$, que minimiza a função objetivo $J(p)$ (Figura 2.3).

\section{Região de convergência do algoritmo de ajuste}

Define-se como região de convergência do algoritmo de ajuste, ou simplesmente região de convergência, ao espaço de parâmetros na qual o algoritmo de ajuste garante a estimação do vetor de parâmetros verdadeiro $p^{*}$.

Na Figura 2.3, por exemplo, considerando como valor inicial o vetor de parâmetros $p_{1}$, dentro da região de convergência, observa-se que o algoritmo consegue encontrar o valor verdadeiro $p^{*}$, entretanto para um vetor de parâmetros inicial fora da região de convergência, $p_{1 a}$, o algoritmo não consegue encontar o valor verdadeiro. A figura também mostra que pode existir algumas restrições nos parâmetros no momento de escolher o vetor inicial de parâmetros. Em caso do gerador síncrono, por exemplo, os parâmetros devem ser positivos e alguns parâmetros devem ser maiores do que outros (exemplo a reatância de eixo direto deve ser maior que a reatância de eixo em quadratura). O valor inicial dos parâmetros na práticas é obtido a partir de valores nominais do modelo (que pode ter incertezas), dados de sistemas similares, ou, no pior dos casos, uma estimativa que esteja dentro da região factível.

\subsection{Funções de Sensibilidade de Trajetória}

No caso de modelos estáticos, a verificação das condições de otimalidade e a implementação de algoritmos para encontrar os parâmetros que minimiza a função objetivo requer o cálculo de derivadas de funções algébricas não-lineares. No caso 


\section{Região factível}

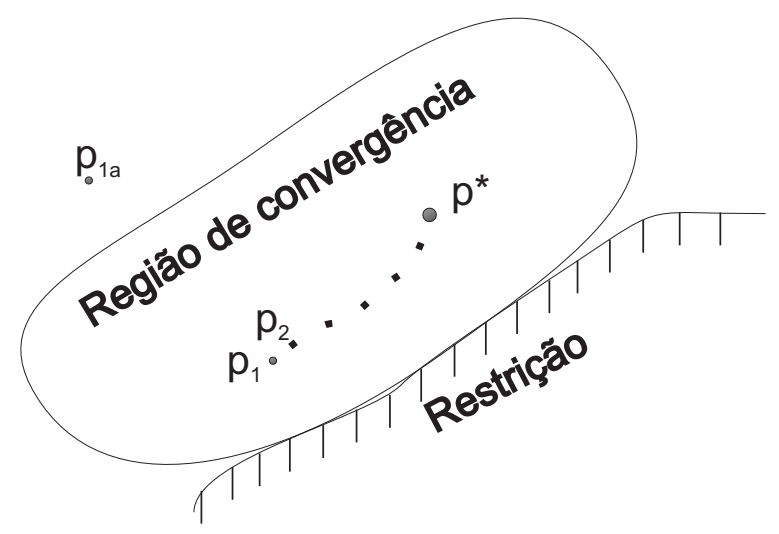

Figura 2.3: Região de estimação do algoritmo de ajuste.

de sistemas dinâmicos, estas derivadas são denominadas funções de sensibilidade de trajetória. Estas funções quase sempre são obtidas por processamento numérico e apenas em casos muito especiais podem ser obtidas explicitamente em termos de funções elementares.

Em sistemas de potência as funções de sensibilidade de trajetória tem-se usado para estimação de parâmetros (Sanchez et al., 1988; Benchluch e Chow, 1993; Hiskens e Koeman, 1998) e análise de estabilidade dinâmica (Hiskens, 2001; Ferreira et al., 2004). O método de estimação de parâmetros baseado em sensibilidade de trajetória pode ser facilmente implementado para uma grande classe de sistemas não-lineares incluindo não linearidades complexas. Em (Hiskens, 2001), por exemplo, apresenta-se uma interessante extensão da análise de sensibilidade para equações algébrico-diferenciais (EAD's) sujeitas a eventos não-suaves, tais como chaveamentos.

Esta seção apresenta o procedimento para o cálculo das funções de sensibilidade de trajetória para estimação de parâmetros de sistemas lineares e nãolineares (Sanchez et al., 1988), (Benchluch e Chow, 1993).

As funções de sensibilidade de trajetória quantificam a variação da solução de equações diferenciais com relação à variação de seus parâmetros (Cruz, 1972).

Seja $y(t, p)$ o vetor solução de uma equação diferencial ordinária dependente do vetor de parâmetros $p$; a variação $\Delta y(t, p)$, devido à variação $\Delta p$, pode ser 
aproximada por:

$$
\Delta y(t, p) \approx \sum_{i}^{n} \frac{\partial y}{\partial p_{i}} \Delta p_{i}
$$

As derivadas parciais

$$
\frac{\partial y}{\partial p_{i}} \doteq \lambda_{y}^{p_{i}}
$$

são chamadas de funções de sensibilidade de trajetória e descrevem os efeitos da variação dos parâmetros na solução da equação diferencial.

A matriz de sensibilidade $\frac{\partial y}{\partial p}$ é a matriz cujas colunas correspondem às derivadas parciais da saída em relação a cada parâmetro $p_{i}$. Esta matriz pode ser utilizada para verificar se o conjunto de medidas contém informação suficiente para estimar os parâmetros. Para isto, avaliam-se os valores singulares da matriz de sensibilidade. A relação entre o menor e o maior valor singular mede o grau de condicionamento numérico do processo de estimação (Stewart, 1973; Aguirre, 1995).

Para interpretar o significado das funções de sensibilidade de trajetória, a seguir analisa-se o sistema massa-mola apresentado na Seção 2.1.

Exemplo 2.2 Seja o sistema massa-mola descrito no Exemplo 2.1 com condições iniciais $x_{1}(0)=0, x_{2}(0)=0$. Os parâmetros nominais deste sistema são $k=8$ e $m=2$, a entrada é uma função degrau de amplitude $u_{o}=4$.

A saída y e as duas funções de sensibilidade $\frac{\partial y}{\partial k}$ e $\frac{\partial y}{\partial m}$ são mostradas na Figura 2.4. Algumas observações importantes podem ser deduzidas analisando estas funções. Por exemplo, $\frac{\partial y}{\partial m}$ indica que o parâmetro $m$ influencia mais a resposta do sistema que o parâmetro $k$ (a amplitude de $\frac{\partial y}{\partial m}$ é maior do que $\frac{\partial y}{\partial k}$ ). Além disso, as mudanças quantitativas de y provocadas por variações paramétricas podem ser estimadas facilmente a partir destas funções usando (2.3),

$$
\Delta y=\frac{\partial y}{\partial k} \Delta k+\frac{\partial y}{\partial m} \Delta m .
$$




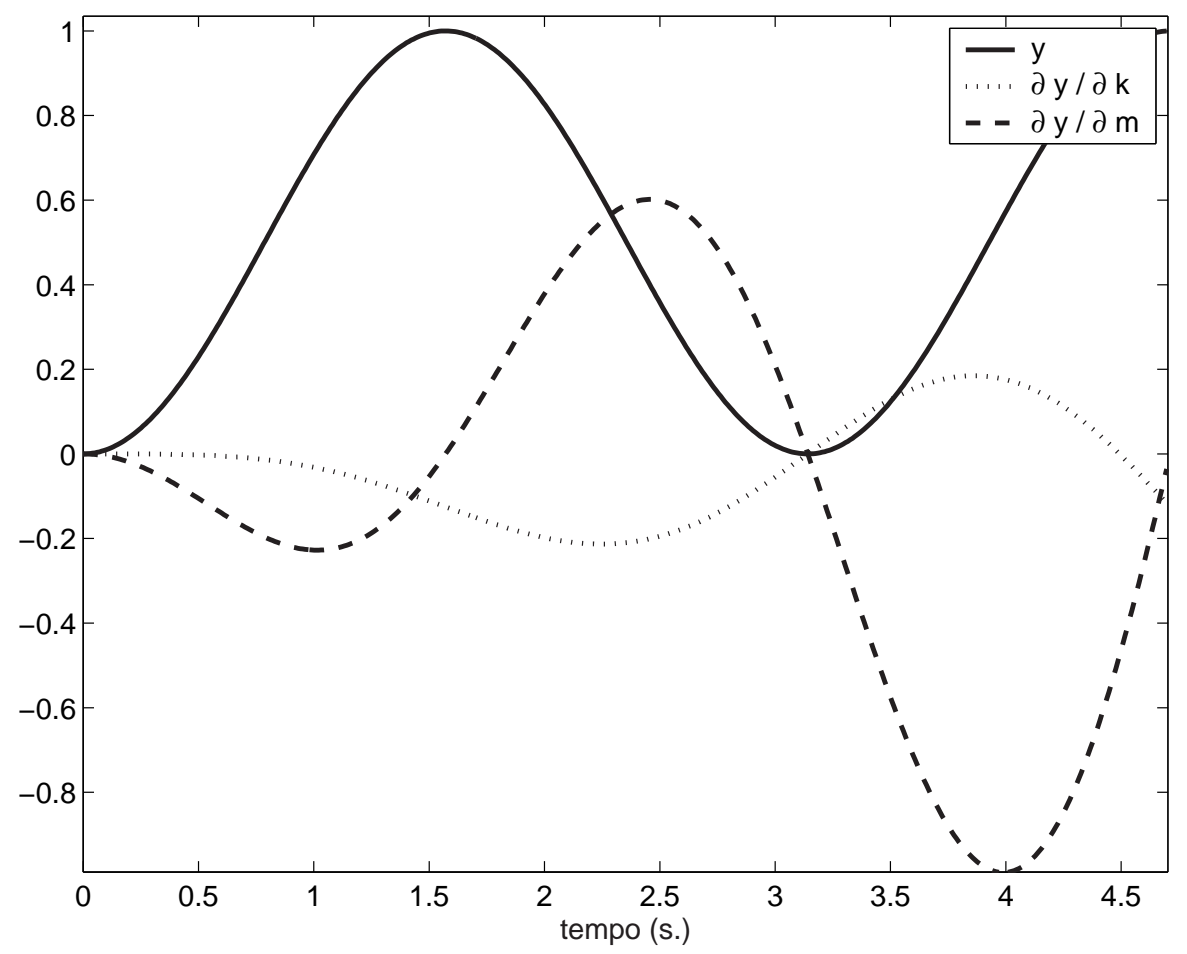

Figura 2.4: Saída e funções de sensibilidade para o Exemplo 2.2

As funções de sensibilidade para sistemas lineares invariantes no tempo podem ser encontradas resolvendo-se explicitamente o sistema linear e derivandose parcialmente a solução em relação a cada parâmetro. Este procedimento é complicado mesmo em sistemas de baixa ordem e quase sempre impossível para sistemas variantes no tempo e sistemas não-lineares, pois raramente é possível expressar a solução de equações diferenciais ordinárias em termos de funções elementares conhecidas ${ }^{1}$. Para contornar este problema, as funções de sensibilidade de trajetória são obtidas numericamente a partir de equações diferenciais obtidas do modelo o qual denomina-se equações de sensibilidade. Nas próximas seções, mostra-se o procedimento para determinar as equações de sensibilidade, primeiro para sistemas lineares invariantes no tempo e depois para sistemas não-lineares.

\footnotetext{
${ }^{1}$ Como ilustração, as funções de sensibilidade de trajetória foram obtidas literalmente para o sistema massa-mola em (Cari, 2005).
} 


\subsubsection{Funções de sensibilidade de trajetória de sistemas lineares invariantes no tempo (LIT)}

Suponha que o sistema linear invariante no tempo dependente do vetor de parâmetros $p$ seja descrito por:

$$
\begin{aligned}
\frac{d}{d t} x(t) & =A(p) x(t)+B(p) u(t), \\
y(t) & =C(p) x(t)
\end{aligned}
$$

em que $x \in R^{m}$ é o vetor de estados, $y \in R^{r}$ é o vetor de saída, $u \in R^{l}$ é o vetor de entrada, $p \in R^{p}$ é o vetor de parâmetros e $A(p), B(p), C(p)$ matrizes que dependem do vetor de parâmetros. A condição inicial é $x\left(t_{o}\right)=x_{o}$.

As equações de sensibilidade para as variáveis de estado podem ser determinadas derivando-se (2.6) em relação ao parâmetro $p_{i}$,

$$
\frac{d}{d t}\left(\frac{\partial x(t)}{\partial p_{i}}\right)=A(p) \frac{\partial x(t)}{\partial p_{i}}+\frac{\partial A(p)}{\partial p_{i}} x(t)+\frac{\partial B(p)}{\partial p_{i}} u(t) .
$$

As equações de sensibilidade da saída podem ser determinadas derivando-se (2.7) com relação ao parâmetro $p_{i}$ :

$$
\frac{\partial y}{\partial p_{i}}=\frac{\partial C(p)}{\partial p_{i}} x(t)+C(p) \frac{\partial x(t)}{\partial p_{i}} .
$$

A condição inicial para a equação (2.8) pode ser encontrada por:

$$
\left.\frac{\partial x(t)}{\partial p_{i}}\right|_{t=t o}=\frac{\partial x_{o}}{\partial p_{i}}
$$

As equações (2.8) e (2.9) são equações diferenciais lineares nas variáveis $\frac{\partial x}{\partial p} \mathrm{e}$ $\frac{\partial y}{\partial p}$. Este conjunto de equações é denominado modelo de sensibilidade.

A solução de (2.8) e (2.9) exige o conhecimento da solução das equações (2.6) e (2.7), as quais podem ser obtidas por integração numérica.

Exemplo 2.3 Considere o sistema de equações diferenciais do sistema massamola, as equações de sensibilidade para o sistema massa-mola são determinadas derivando o sistema de equações 2.2 em relação ao vetor de parâmetros $p=$ $(k, m)^{T}$. 
Em relação ao parâmetro $k$ :

$$
\begin{aligned}
\dot{\lambda}_{x_{1}}^{k} & =\lambda_{x_{2}}^{k}, \\
\dot{\lambda}_{x_{2}}^{k} & =-\frac{k}{m} \lambda_{x_{1}}^{k}-\frac{1}{m} x_{1}, \\
\lambda_{y}^{k} & =\lambda_{x_{1}}^{k},
\end{aligned}
$$

sendo $\lambda_{x_{1}}^{k}=\frac{\partial x_{1}}{\partial k}, \lambda_{x_{2}}^{k}=\frac{\partial x_{2}}{\partial k}$ e $\lambda_{y}^{k}=\frac{\partial y}{\partial k}$.

Em relação ao parâmetro $m$ :

$$
\begin{aligned}
\dot{\lambda}_{x_{1}}^{m} & =\lambda_{x_{2}}^{m}, \\
\dot{\lambda}_{x_{2}}^{m} & =-\frac{1}{m^{2}} u(t)-\frac{k}{m} \lambda_{x_{1}}^{m}+\frac{k}{m^{2}} x_{1}, \\
\lambda_{y}^{m} & =\lambda_{x_{1}}^{m}
\end{aligned}
$$

sendo $\lambda_{x_{1}}^{m}=\frac{\partial x_{1}}{\partial m}, \lambda_{x_{2}}^{m}=\frac{\partial x_{2}}{\partial m}$ e $\lambda_{y}^{m}=\frac{\partial y}{\partial m}$.

\subsubsection{Funções de sensibilidade de trajetória de sistemas dinâmicos não-lineares}

Nesta seção, apresenta-se a obtenção das funções de sensibilidade de trajetória para sistemas dinâmicos não-lineares modelados por equações algébricadiferenciais (EAD) segundo a formulação tradicional (Sanchez et al., 1988; Benchluch e Chow, 1993; Hiskens e Koeman, 1998). A obtenção das funções de sensibilidade depende da solução das equações EADs do modelo, por isso, no começo será abordada a forma de resolver as EADs do modelo.

Considere um sistema dinâmico não-linear modelado por

$$
\begin{aligned}
\dot{x} & =f(x, z, p, u), \\
0 & =g(x, z, p, u), \\
y & =h(x, z, p, u),
\end{aligned}
$$

em que $x \in R^{m}$ é o vetor de estados, $z \in R^{q}$ é o vetor de variáveis algébricas, $y \in R^{r}$ é o vetor de saída, $u \in R^{l}$ é o vetor de entrada e $p \in R^{p}$ é o vetor de parâmetros. Seja $p_{i}$ a $i$-ésima componente de ' $p$ '. Admite-se que as funções $f, g$ e $h$ são diferenciáveis em relação a cada $p_{i}, i=1, \ldots, p$. 
O sistema (2.13) pode ser resolvido por um método de integração implícito, tal como a regra trapezoidal. Para este objetivo, expressa-se o sistema (2.13) pelo seguinte sistema discreto.

$$
\begin{gathered}
\left\{\begin{array}{c}
x_{n+1}-x_{n}-\frac{\Delta t}{2} f\left(x_{n+1}, z_{n+1}, p, u_{n+1}\right)-\frac{\Delta t}{2} f\left(x_{n}, z_{n}, p, u_{n}\right)=0 \\
g\left(x_{n+1}, z_{n+1}, p, u_{n+1}\right)=0
\end{array}\right. \\
y_{n+1}=h\left(x_{n+1}, z_{n+1}, p, u_{n+1}\right)
\end{gathered}
$$

em que os sub-índices " $n$ " e " $n+1$ " representam variáveis nos instantes $t_{n}=t_{o}+n \Delta t$ e $t_{n+1}=t_{o}+(n+1) \Delta t$, respectivamente, e $\Delta t$ é o passo de integração.

O sistema algébrico não-linear equivalente (2.14) pode ser resolvido usando o método de Newton. Este procedimento deve ser realizado para todo instante de tempo $t_{n}$ dentro do intervalo de integração. A saída $y_{n+1}$ pode ser determinada por substituição direta das variáveis encontradas $x_{n+1}$ e $z_{n+1}$ na equação (2.15). No final do processo de integração as variáveis $x(t), z(t)$ e $y(t)$ estarão disponíveis. O procedimento para resolver (2.13) pelo método implícito pode ser dividido nas seguintes etapas.

a) Defina as EADs do modelo (2.13) e as condições iniciais $t_{o}, x_{o}, z_{o}$ e faça $n=1$.

b) Transforme as EADs em um conjunto de equações algébricas não-lineares usando a regra trapezoidal (2.14) $-(2.15)$.

c) Faça $t_{n}=t_{o}+n \Delta t$.

d) Resolva o sistema (2.14) pelo método de Newton nas variáveis $x_{n+1}, z_{n+1}$, considerando como estimativas iniciais $x_{n+1}=x_{n}$ e $z_{n+1}=z_{n}$.

e) Determine a saída $y_{n+1}$ por substituição direta das variáveis obtidas $x_{n+1}$ e $z_{n+1}$. Armazene estas variáveis.

f) Pare se $t_{n+1}>t_{\max }$ (intervalo total das medidas), caso contrário faça $n=$ $n+1$ e vá para o passo c. 


\section{Obtenção das funções de sensibilidade de trajetória}

Derivando-se (2.13) com relação a cada parâmetro $p_{i}$, obtêm-se as equações de sensibilidade:

$$
\begin{aligned}
\frac{d}{d t} \frac{\partial x}{\partial p_{i}} & =\frac{\partial f(x, z, p, u)}{\partial x} \cdot \frac{\partial x}{\partial p_{i}}+\frac{\partial f(x, z, p, u)}{\partial z} \cdot \frac{\partial z}{\partial p_{i}}+\frac{\partial f(x, z, p, u)}{\partial p_{i}} \\
0 & =\frac{\partial g(x, z, p, u)}{\partial x} \cdot \frac{\partial x(t)}{\partial p_{i}}+\frac{\partial g(x, z, p, u)}{\partial z} \cdot \frac{\partial z(t)}{\partial p_{i}}+\frac{\partial g(x, z, p, u)}{\partial p_{i}} \\
\frac{\partial y}{\partial p_{i}} & =\frac{\partial h(x, z, p, u)}{\partial x} \cdot \frac{\partial x}{\partial p_{i}}+\frac{\partial h(x, z, p, u)}{\partial z} \cdot \frac{\partial z}{\partial p_{i}}+\frac{\partial h(x, z, p, u)}{\partial p_{i}}
\end{aligned}
$$

O sistema (2.16) é não-linear e, em geral, não possui solução em termos de funções elementares conhecidas e, portanto, deve ser resolvido usando um método de integração implícito da mesma forma como o desenvolvido para a solução das EADs do modelo.

O sistema (2.13) não depende da solução de (2.16), porém o sistema (2.16) depende da solução de (2.13). Geralmente (2.13) é resolvido primeiro e sua solução é usada para resolver (2.16). Uma grande vantagem pode ser obtida resolvendo (2.13) e (2.16) em forma alternada por um algoritmo implícito de integração numérica. Pois, neste caso, os dois sistemas possuem a mesma matriz Jacobiana em cada passo de integração o que acelera o processo de cálculo. No final do intervalo de integração, as sensibilidades $\frac{\partial x}{\partial p_{i}}, \frac{\partial z}{\partial p_{i}}$ e $\frac{\partial y}{\partial p_{i}}$ estarão disponíveis.

Se $f, g$ ou $h$ não forem diferenciáveis com relação ao parâmetro $p_{i}$, as sensibilidades podem ser aproximadas da seguinte maneira (Benchluch e Chow, 1993). Considere $p^{o}$ o vetor de parâmetros inicial e seja $p^{1}$ um vetor cuja $i$-ésima componente é $p_{i}^{1}=p_{i}^{o}+\Delta p_{i}$, em que $\Delta p_{i}$ é uma pequena perturbação no parâmetro. Seja $x_{o}(t), z_{o}(t)$ e $y_{o}(t)$ a resposta no tempo de (2.13) obtida com o parâmetro $p_{o}$ e seja $x_{1}(t)$ e $z_{1}(t), y_{1}(t)$ a resposta no tempo obtida com o parâmetro $p_{1}$. Desta forma, as funções de sensibilidade de trajetórias podem ser aproximadas por:

$$
\begin{aligned}
\frac{\partial x(t)}{\partial p_{i}} & \approx \frac{x_{1}(t)-x_{o}(t)}{\Delta p_{i}}, \\
\frac{\partial z(t)}{\partial p_{i}} & \approx \frac{z_{1}(t)-z_{o}(t)}{\Delta p_{i}} \\
\frac{\partial y(t)}{\partial p_{i}} & \approx \frac{y_{1}(t)-y_{o}(t)}{\Delta p_{i}} .
\end{aligned}
$$

Uma grande dificuldade para obter a solução de (2.13) com a abordagem desta 
seção acontece quando as equações algébricas do modelo não possuem solução. Se a estimativa inicial de $p$ estiver distante do valor verdadeiro, a solução da equação algébrica pode não existir. Como conseqüência, as funções sensibilidades de trajetória não poderiam ser calculadas e os parâmetros não poderão ser estimados. A fim de evitar este problema, na seqüência propõe-se uma abordagem que modela as equações algébrica-diferenciais como um problema de minimização.

\subsubsection{Funções de sensibilidade de trajetória para sistemas algébrico-diferenciais: Abordagem de minimização}

Com o objetivo de evitar o problema de inexistência de solução da equação algébrica das EADs do modelo, propõe-se neste trabalho uma nova abordagem de solução das EADs por médio de um problema de minimização restrito. Esta abordagem relaxa a restrição de igualdade das EADs durante o processo de integraçãa ${ }^{2}$ e permite a obtenção de soluções quando as EADs possuem singularidades. Sendo Assim, esta abordagem de solução contém a solução das EADs com o um caso particular. Matematicamente (2.13) é substituído por:

$$
\begin{aligned}
& \min _{(x, z)} \sum_{i} g_{i}^{2}(x, z, p, u) \\
& \text { s.a. } \quad\{\quad \dot{x}=f(x, z, p, u)\}, \\
& y=h(x, z, p, u),
\end{aligned}
$$

em que $g_{i}$ representa a $i$-ésima equação algébrica do modelo (2.13), a equação $\dot{x}=f(x, z, p, u)$ é uma restrição e $y$ é a saída. O mínimo global esperado para este problema de minimização é zero. Quando o mínimo global é alcançado, $g_{i}=0$ para todo $i$, a solução de (2.18) coincide com a solução do modelo (2.13). Após a convergência de $p$, precisa-se verificar se o mínimo global foi alcançado para validar a estimação dos parâmetros.

\footnotetext{
${ }^{2}$ Uma outra possibilidade poderia ter sido relaxar a equação algébrica por uma restrição de desigualdade do tipo $g(x, z, u, p)<\epsilon$, com $0<\epsilon<1$. Entretanto, a desigualdade na equação algébrica não permite a obtenção das funções de sensibilidades que dependem das derivadas parciais das EADs do modelo em relação a cada parâmetro.
} 
A forma discreta de (2.18) pode ser obtida usando um método de integração implícito tal como a regra trapezoidal, isto é:

$$
\begin{aligned}
& \min _{x_{n+1}, z_{n+1}} \quad \sum_{i} g_{i}^{2}\left(x_{n+1}, z_{n+1}, p, u_{n+1}\right) \\
& \text { s.a }\left\{x_{n+1}-x_{n}-\frac{\Delta t}{2}\left[f\left(x_{n}, z_{n}, p, u_{n}\right)+\right.\right. \\
& \left.\left.f\left(x_{n+1}, z_{n+1}, p, u_{n+1}\right)\right]\left.\right|_{j}=0\right\}, \\
& y_{n+1}=h\left(x_{n+1}, z_{n+1}, p, u_{n+1}\right) \text {, }
\end{aligned}
$$

em que o sub-íncice "j” representa a j-ésima equação diferencial. Os sub-índices n e $n+1$ denotam os valores das variáveis nos tempos $t_{n}=t_{o}+n \Delta t \mathrm{e}$ $t_{n+1}=t_{o}+(n+1) \Delta t$, respectivamente.

O método de multiplicadores de Lagrange (Bazaraa, 1979) pode ser usado para resolver o problema de minimização restrito (2.19). A função Lagrangeana não-restrita, a qual depende das variáveis $\left(x_{n+1}, z_{n+1}\right)$ e do multiplicador de Lagrange $\left(\lambda_{L(n+1)}\right)$, é dada por:

$$
L=\sum_{i} g_{i}^{2}\left(x_{n+1}, z_{n+1}, p, u_{n+1}\right)+\sum_{j} \lambda_{L n+1}\left[\begin{array}{l}
x_{n+1}-x_{n}-\frac{\Delta t}{2}\left(f\left(x_{n}, z_{n}, p, u_{n}\right)+\right. \\
\left.\left.f\left(x_{n+1}, z_{n+1}, p, u_{n+1}\right)\right)\right]\left.\right|_{j} \cdot(2.20)
\end{array}\right.
$$

As condições de otimalidade de KKT (Karush-Kuhn-Tucker) (Bazaraa, 1979) são:

$$
\begin{aligned}
\frac{\partial L}{\partial x_{n+1}} & =2 g_{n+1} \frac{\partial g_{n+1}}{\partial x_{n+1}}+\lambda_{L(n+1)}\left(1-\frac{\Delta t}{2} \frac{\partial f_{n+1}}{\partial x_{n+1}}\right)=0 \\
\frac{\partial L}{\partial z_{n+1}} & =2 g_{n+1} \frac{\partial g_{n+1}}{\partial z_{n+1}}+\lambda_{L(n+1)}\left(-\frac{\Delta t}{2}\right) \frac{\partial f_{n+1}}{\partial z_{n+1}}=0 \\
\frac{\partial L}{\partial \lambda_{L n+1}} & =x_{n+1}-x_{n}-\frac{\Delta t}{2}\left(f_{n}+f_{n+1}\right)=0
\end{aligned}
$$

em que $g_{n+1}=g\left(x_{n+1}, z_{n+1}, p, u_{n+1}\right), \quad f_{n}=f\left(x_{n}, z_{n}, p, u_{n}\right) \quad$ e $f_{n+1}=f\left(x_{n+1}, z_{n+1}, p, u_{n+1}\right)$. Resolver (2.21), consiste em encontrar as raízes de um sistema de equações não lineares, o qual pode ser resolvido pelo método de Newton. Para isto, faz-se:

$$
F_{L(n+1)}:=\left(\frac{\partial L}{\partial x_{n+1}}, \frac{\partial_{L}}{\partial z_{n+1}}, \frac{\partial L}{\partial \lambda_{L n+1}}\right)^{T}=0 .
$$


Partindo de uma estimativa inicial $v_{n}=\left(x_{n}, z_{n}, \lambda_{L n}\right)$ o método de Newton estima a solução $v_{n+1}=\left(x_{n+1}, z_{n+1}, \lambda_{L n+1}\right)$. Isto é realizado em cada instante de tempo até o final do intervalo de integração.

O algoritmo para a solução das equações do modelo (2.13) pela abordagem de minimização utilizando o método implícito pode ser resumido nos seguintes passos:

a) Transforme as EADs do modelo pelo modelo de minimização discretizado (2.19), defina as condições inicias $t_{o}, x_{o}, z_{o}$ e faça $n=1$;

b) Encontre a função Lagrangeana (2.20);

c) Determine as derivadas parciais da função Lagrangeana em relação às variáveis $x_{n+1}, z_{n+1}$ e $\lambda_{L n+1}, F_{L}(2.22)$

d) Faça $t_{n}=t_{o}+n \Delta t$.

e) Resolva o sistema (2.22) pelo método de Newton nas variáveis $x_{n+1}, z_{n+1}$ e $\lambda_{L n+1}$, considerando como estimativa inicial $x_{n+1}=x_{n}, z_{n+1}=z_{n}$ e $\lambda_{L n+1}=\lambda_{L n}$

f) Determine a saída $y_{n+1}$ por substituição direta das variáveis $x_{n+1}$ e $z_{n+1}$. Armazene estas variáveis.

g) Pare se $t_{n+1}>t_{\max }$, caso contrário faça $n=n+1$ e vá para o passo c. .

No final do intervalo de integração, as variáveis do modelo $x(t), z(t)$ e $y(t)$ serão determinadas. 
Obtenção das equações de sensibilidade para a modelagem de minimização

As sensibilidades podem ser determinadas derivando parcialmente (2.21) em relação a cada parâmetro. Como ilustração, para o parâmetro $p_{i}$, tem-se:

$$
\begin{aligned}
2\left(\frac{\partial g_{n+1}}{\partial p_{i}} \frac{\partial g_{n+1}}{\partial x_{n+1}}+g_{n+1} \frac{\partial}{\partial p_{i}}\left(\frac{\partial g_{n+1}}{\partial x_{n+1}}\right)\right)-\lambda_{L n+1} \frac{\Delta t}{2} \frac{\partial}{\partial p_{i}}\left(\frac{\partial f_{n+1}}{\partial x_{n+1}}\right) & =0 \\
2\left(\frac{\partial g_{n+1}}{\partial p_{i}} \frac{\partial g_{n+1}}{\partial z_{n+1}}+g_{n+1} \frac{\partial}{\partial p_{i}}\left(\frac{\partial g_{n+1}}{\partial z_{n+1}}\right)\right)-\lambda_{L n+1} \frac{\Delta t}{2} \frac{\partial}{\partial p_{i}}\left(\frac{\partial f_{n+1}}{\partial z_{n+1}}\right) & =0 \\
\frac{\partial x_{n+1}}{\partial p_{i}}-\frac{\Delta t}{2}\left(\frac{\partial f_{n}}{\partial p_{i}}+\frac{\partial f_{n+1}}{\partial p_{i}}\right) & =0 .
\end{aligned}
$$

O conjunto de equações resultante pode ser solucionado, aplicando-se o método de Newton para encontrar as raízes do sistema de equações não lineares. Em cada passo de integração, determinam-se $\frac{\partial x_{n+1}}{\partial p_{i}}, \frac{\partial z_{n+1}}{\partial p_{i}}$ e $\frac{\partial \lambda_{L(n+1)}}{\partial p_{i}}$. No final do tempo amostrado, as funções de sensibilidade $\frac{\partial x(t)}{\partial p_{i}}$ e $\frac{\partial y(t)}{\partial p_{i}}$ serão determinadas.

\subsection{Metodologia de estimação de parâmetros ba- seado em sensibilidade de trajetória}

A estimação de parâmetros é formulado como um problema de otimização não-linear conforme descrito na Seção 2.4. Para fins didáticos, a partir deste ponto o modelo matemático será denominado sistema auxiliar.

Define-se a função objetivo $J(p)$, que mede a proximidade entre as saídas do sistema real e do sistema auxiliar, como

$$
J(p)=\frac{1}{2} \int_{0}^{T_{o}}\left(y_{\text {med }}-y\right)^{T}\left(y_{\text {med }}-y\right) d t
$$

sendo $y_{\text {med }}$ o vetor de saída do sistema real, y a saída do sistema auxiliar (2.13) e $\left[0, T_{o}\right]$ o intervalo de tempo em análise. O fator $\frac{1}{2}$ que aparece na Equação (2.24) é utilizado apenas para simplificar os cálculos.

As condições de otimalidade estudadas na Seção 2.4 poderiam ser verificadas diretamente se a função objetivo fosse conhecida em forma explícita, entretanto devido à saída do sistema auxiliar y ser calculada numericamente, as condições de otimalidade só podem ser verificadas numericamente. 
O mínimo de (2.24) pode ser encontrado aplicando-se a primeira condição de otimalidade para o problema irrestrito, isto é, $\nabla J(p *)=0$. A segunda condição de otimalidade (Hessiana definida positiva) não será verificada devido à complexidade dos cálculos. Assim

$$
G(p)=-\frac{\partial J(p)}{\partial p}=\int_{0}^{T_{o}} \frac{\partial y^{T}}{\partial p}\left(y_{m e d}-y\right) d t .
$$

O método de Newton pode ser usado para resolver a equação não linear (2.25). Partindo do vetor inicial $p=p^{(o)}$, o ajuste dos parâmetros na $k$-ésima iteração é dado por:

$$
p^{(k+1)}=p^{(k)}-\left.\Gamma^{-1}(p) G(p)\right|_{p=p^{(k)}},
$$

sendo $\Gamma(p)$ a matriz Jacobiana de $G(p)$, obtida derivando-se $G(p)$ em relação ao vetor de parâmetros $p$

$$
\Gamma(p):=\frac{\partial G(p)}{\partial p}=-\int_{0}^{T_{o}} \frac{\partial^{2} y^{T}}{\partial p^{2}}\left(y_{m e d}-y\right) d t+\left.\int_{0}^{T_{o}}\left(\frac{\partial y}{\partial p}\right)^{T}\left(\frac{\partial y}{\partial p}\right) d t\right|_{p=p^{(k)}} .
$$

Desprezando os termos de segunda ordem, $\Gamma(p)$ pode ser aproximada por:

$$
\left.\Gamma(p) \approx \int_{0}^{T_{o}}\left(\frac{\partial y}{\partial p}\right)^{T}\left(\frac{\partial y}{\partial p}\right) d t\right|_{p=p^{(k)}} .
$$

Para implementação, a saída $y_{m e d}$ do sistema real é amostrada em intervalos regulares de tempo, neste caso, as integrais são substituídas por somatórios nas equações (2.25) e (2.27). Observa-se que tanto $G(p)$ como $\Gamma(p)$ dependem do cálculo das funções de sensibilidade de trajetória. Para maiores detalhes veja (Cruz, 1972).

A seguir apresenta-se o algoritmo de ajuste de parâmetros considerando a abordagem tradicional (Seção 2.5.2) e a abordagem de minimização proposta (Seção 2.5.3).

\subsubsection{Algoritmo de estimação de parâmetros com a for- mulação tradicional}

O ajuste dos parâmetros baseado na sensibilidade de trajetória com a formulação tradicional modela o sistema auxiliar por EADs (2.13), e obtém as 
funções de sensibilidade de trajetória diretamente a partir das EADs do sistema auxiliar (2.16) por diferenciação. Os passos da metodologia de sensibilidade de trajetória com a formulação tradicional são:

a. Selecione a estrutura do modelo e os parâmetros a serem estimados.

b. Escolha uma estimativa inicial $p^{(o)}=p_{o}$ para os parâmetros e faça $k=0$.

c. Use os dados medidos para calcular a saída do sistema auxiliar a partir de (2.13) via integração numérica.

d. Compare as saídas do sistema real e do modelo por intermédio de $J\left(p^{(k)}\right)$.

e. Pare se $J\left(p^{(k)}\right)$ for menor do que uma tolerância ou se não mudar significativamente de uma iteração para outra, caso contrário vá para o passo f.

f. Use as entradas medidas para calcular funções de sensibilidade a partir de (2.16) via integração implícita.

g. Calcule $G\left(p^{(k)}\right)$ e $\Gamma\left(p^{(k)}\right)$ a partir de $(2.25)$ e (2.27), respectivamente.

h. Atualize os parâmetros usando a equação (2.26), faça $k=k+1$ e vá para o passo c.

\subsubsection{Algoritmo de estimação de parâmetros com a abor- dagem de minimização}

Nesta abordagem, as EADs do modelo são substituídas por um problema de minimização a partir de(2.18) e as funções de sensibilidade a partir de (2.23). Os passos da metodologia de sensibilidade de trajetória com a abordagem de minimização são:

a. Selecione a estrutura do modelo e os parâmetros a serem estimados.

b. Escolha uma estimativa inicial $p^{(o)}=p_{o}$ para os parâmetros e faça $k=0$.

c. Modele o sistema auxiliar pelo problema de minimização (2.19) e calcule as saídas do sistema auxiliar a partir da solução de (2.21). 
d. Compare as saídas do sistema real e do sistema auxiliar por intermédio de $J\left(p^{(k)}\right)$.

e. Pare se a equação algébrica $g($.$) for nula para todo n$ e se $J\left(p^{(k)}\right)$ for menor do que uma tolerância ou não tiver mudança significativa de uma iteração para outra, caso contrário vá para "f".

f. Resolva as equações (2.23) pelo método de Newton para obter as funções de sensibilidade de trajetória.

g. Calcule $G\left(p^{(k)}\right)$ e $\Gamma\left(p^{(k)}\right)$ a partir de $(2.25)$ e (2.27), respectivamente.

h. Atualize os parâmetros usando a equação (2.26), faça $k=k+1$ e vá para o passo c.

\subsection{Estimação de Parâmetros em Duas Fases}

Durante o processo de estimação pode ocorrer divergência dos parâmetros especialmente quando vários parâmetros são estimados simultaneamente. Um dos principais fatores que contribuem para que isto aconteça é que a influência sobre a saída de alguns parâmetros é muito pequeno comparado com a influência dos outros parâmetros do sistema auxiliar o que torna a matriz $\Gamma$ mal-condicionada. O ajuste de parâmetros fornecida pela metodologia de estimação torna-se muito crítica nos parâmetros com menor influência da saída, produzindo grandes variações nestes parâmetros o que pode levar à divergência dos parâmetros estimados ou à convergência a parâmetros não-realísticos (por exemplo parâmetros negativos que não tem significado físico). As metodologias de estimação de parâmetros, neste caso, apenas garantem a correta estimação quando os vetor inicial de parâmetros estiver muito próximo do seu valor verdadeiro. Isto limita a região de convergência e torna as metodologias de estimação inviáveis em sistemas reais.

A fim de aumentar a região de convergência, realiza-se uma análise de sensibilidade para determinar os parâmetros que têm menos influência sobre a saída 
e alterar o processo de ajuste destes parâmetros.

\subsubsection{Classificação dos parâmetros segundo sua influência na saída do modelo}

Uma forma de determinar a influência dos parâmetros na saída (menor sensibilidade) é a decomposição QR da matriz $\Gamma$ (Burth et al., 1999). O algoritmo é como segue:

a. Dado a estimativa inicial dos parâmetros $p=p_{o}$, determine $\Gamma$ e calcule a decomposição em autovetores e autovalores: $\Gamma=U V U^{T}$.

b. Determine $\rho$, tal que os $\rho$ autovalores de $\Gamma$ sejam maiores que os restantes, $n-\rho$ autovalores, e faça uma partição $U=\left[U_{\rho} U_{n-\rho}\right]$ com $U_{\rho}$ contendo as primeiras $\rho$ colunas de $\mathrm{U}$.

c. Determine a matriz de permutação $P$ construindo a decomposição $Q R$ considerando como pivô a coluna $U_{\rho}^{T}$ onde, $U_{\rho}^{T} P=Q R$.

d. Reordene o vetor de parâmetros segundo $p=P^{T} p$. Assim, os primeiros $\rho$ elementos de $p$ serão parâmetros bem condicionados.

A classificação dos parâmetros é realizada repetindo-se o procedimento descrito acima variando $\rho$ de 1 até $m-1$, sendo $m$ o número de parâmetros.

\subsubsection{Algoritmo de estimação de parâmetros em duas fa- ses}

Uma forma diminuir os problemas de mal-condicionamento é dividir a estimação de parâmetros em duas fases. Na primeira fase, os parâmetros que produzem maior influência na sensibilidade são atualizados segundo a metodologia de ajuste de parâmetros até que $J(p)$ não mude de uma iteração para outra, enquanto os outros parâmetros são fixados. Na segunda fase, todos os parâmetros são atualizados simultaneamente. Esta abordagem usualmente aumenta a região de 
convergência da metodologia de estimação permitindo a estimação de parâmetros partindo de estimativas iniciais distantes dos valores verdadeiros. O algoritmo é como segue:

a. Dada uma estimativa inicial para os parâmetros, calcule a matriz $\Gamma$.

b. Classifique os parâmetros segundo seu nível de influência na saída usando decomposição $Q R$ e valores singulares.

c. Agrupe os parâmetros com menor sensibilidade no conjunto $p_{b}$ e os outros no conjunto $p_{h}$.

d. Fase 1: Atualize os parâmetros $p_{h}$ mantendo constante o vetor de parâmetros $p_{b}$ utilizando a metodologia de estimação até que $J(p)$ não apresente variação significativa entre uma iteração e a outra (por exemplo uma tolerância de $1 \times 10^{-2}$ ).

e. Fase 2: Atualize todos os parâmetros usando a metodologia de estimação até convergência dos parâmetros. 


\section{Capítulo 3}

\section{Sincronização Mestre-Escravo}

Neste capítulo o processo de estimação de parâmetros é apresentado como um processo de sincronização e o acoplamento "mestre-escravo" (Pecora e Caroll, 1990) é proposto como uma ferramenta para aumentar a região de convergência da metodologia de estimação.

A metodologia de estimação de parâmetros, desenvolvida neste trabalho, está baseada no ajuste iterativo dos parâmetros, através do uso das funções de sensibilidades de trajetória do sistema auxiliar, a fim de minimizar o erro entre as saídas do sistema real e do sistema auxiliar. Isto pode ser entendido como um processo de sincronização de sistemas, no qual a metodologia de estimação ajusta os parâmetros a fim que as saídas dos sistemas real e auxiliar sincronizem, ou seja, fiquem suficientemente próximas em um intervalo finito de tempo. O acoplamento mestre-escravo, o qual consistem em acoplar algumas saídas do sistema mestre (sistema real) com o sistema escravo (sistema auxiliar) em forma unidirecional, ajuda que a sincronização possa ser obtida com maior facilidade.

Usando algumas propriedades de equações diferenciais ordinárias (EDOs) podese provar que se os valores dos parâmetros do sistema auxiliar estiverem suficientemente próximos dos valores verdadeiros implica que há sincronização entre o sistema real e o sistema auxiliar. Entretanto, o inverso não é necessariamente verdade. Um exemplo desta situação pode ser encontrado em (Yu et al., 2007).

Portanto, além de obter a sincronização das saídas por intermédio do ajuste dos parâmetros, deve-se provar que os parâmetros convergiram aos valores ver- 
dadeiros. Esta prova não é trivial para metodologias que utilizam métodos de ajuste iterativos como é o caso da metodologia de sensibilidade de trajetória. Alguns trabalhos preliminares foram gerados nesse sentido (Cari, Theodoro, Bretas e Alberto, 2008; Cari, Theodoro, Mijolaro, Bretas e Alberto, 2009), mas ainda estão na fase de concepção do problema e não foram considerados nesta tese.

Na próxima seção, será apresentada a teoria que justifica a sincronização das saídas para um sistema com dois conjuntos de valores iniciais de parâmetros próximos.

\subsection{Continuidade de Soluções de EDOs}

Duas soluções de um sistema de EDOs com condições inicias e valores de parâmetros suficientemente próximos, ficarão próximas em um intervalo finito de tempo. Esta propriedade baseia-se no seguinte resultado clássico da teoria qualitativa de EDOs.

Teorema 3.1 (Sotomayor, 1979) Suponhamos que $f(t, x, p)$ seja contínua para $(t, x) \in D$ e $p \in V$ onde $V$ é uma vizinhança de $p_{0}$ em $R^{k}$ e $D$ é um conjunto aberto em $R^{n+1}$. Considere o sistema:

$$
\left\{\begin{array}{c}
\dot{x}=f\left(t, x, p_{o}\right) \\
x\left(t_{o}\right)=x_{o} .
\end{array}\right.
$$

sendo $t, x$ e $p_{o}$, o tempo, o vetor de estado e o parâmetro verdadeiro, respectivamente. Se o sistema (3.1) tem uma única solução não continuável $x\left(t, t_{o}, x_{o}, p\right)$ definida em $\left(w_{-}, w_{+}\right)$, então para todo $(s, \eta, p)$ suficientemente próximo de $\left(t_{o}, x_{o}, p_{o}\right)$ o problema de valor inicial (PVI):

$$
\left\{\begin{array}{c}
\dot{x}=f(t, x, p) \\
x(s)=\eta .
\end{array}\right.
$$

tem uma solução $x(t, s, \eta, p)$ definida em $[a, b] \subset\left(w_{-}, w_{+}\right)$que é contínua em $\left(t, t_{o}, x_{o}, p_{o}\right)$.

Considerando apenas continuidade com relação ao parâmetro $p$, pode-se estabelecer o seguinte teorema (Sotomayor, 1979): 
Teorema 3.2 Seja o problema de valor inicial da equação (3.1), dado $\epsilon>0$ arbitrariamente pequeno e $t_{1}>t_{o}, \exists \delta>0$ tal que se $\left\|p-p_{o}\right\|<\delta$, então $\left\|x\left(t, t_{o}, x_{o}, p\right)-x\left(t, t_{o}, x_{o}, p_{o}\right)\right\|<\epsilon$ para todo $t_{o} \leq t<t_{1}$.

As duas soluções $x\left(t, t_{o}, x_{o}, p_{o}\right)$ e $x\left(t, t_{o}, x_{o}, p\right)$ ficarão próximas (dentro do tubo de raio $\epsilon$ ) se os parâmetros $p_{o}$ e $p$ estiverem próximos (dentro da bola de raio $\delta$ ) como mostra a Figura 3.1 .

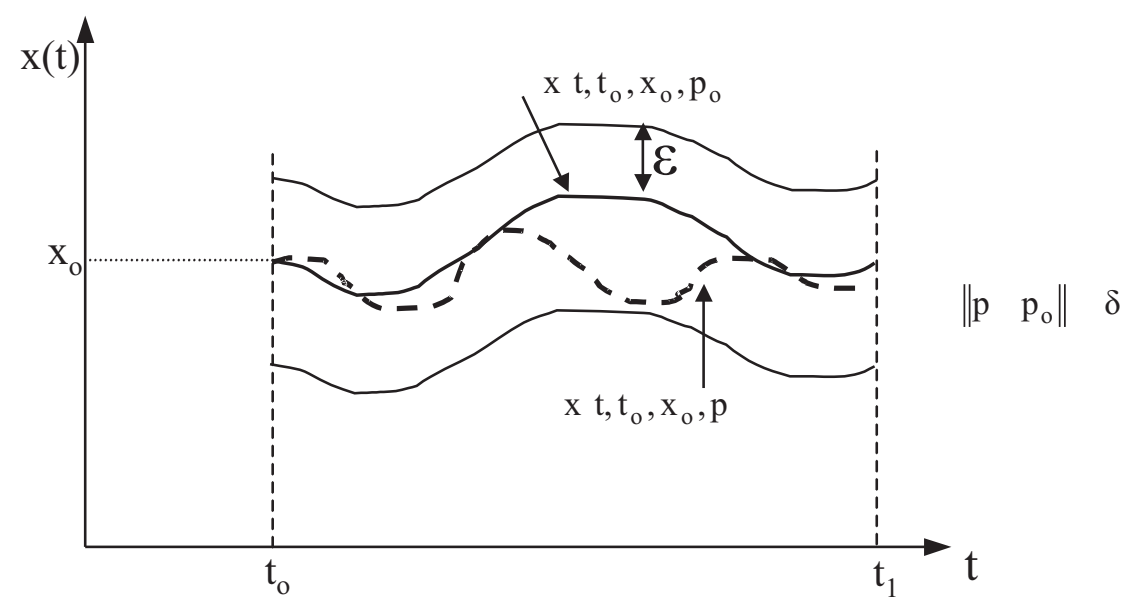

Figura 3.1: Continuidade com relação aos parâmetros.

\subsection{Acoplamento Mestre-Escravo}

Uma alternativa para aumentar a região de convergência é utilizar o acoplamento "mestre-escravo" no processo de estimação (Pecora e Caroll, 1990). Nesta abordagem, o sistema real é o sistema mestre e o sistema auxiliar é o sistema escravo. O acoplamento é do tipo unilateral, isto é, o sistema mestre acopla ao sistema escravo e não viceversa. Na literatura existem relatos de aplicações desta técnica, mas com outros objetivos. Em (Rodrigues, 1996; Gameiro e Rodrigues, 2000) foram desenvolvidos métodos matemáticos usando sincronização de sistemas aplicados a codificação e decodificação de mensagens. Em (Huang e Guo, 2004) a técnica de sincronização foi utilizada para a identificação de parâmetros de equações diferenciais de sistemas caóticos. Na referida pesquisa, os parâmetros foram estimados através de uma função de Lyapunov de controle 
para garantir a sincronização. Na Figura 3.2 compara-se a abordagem com e sem acoplamento mestre-escravo.

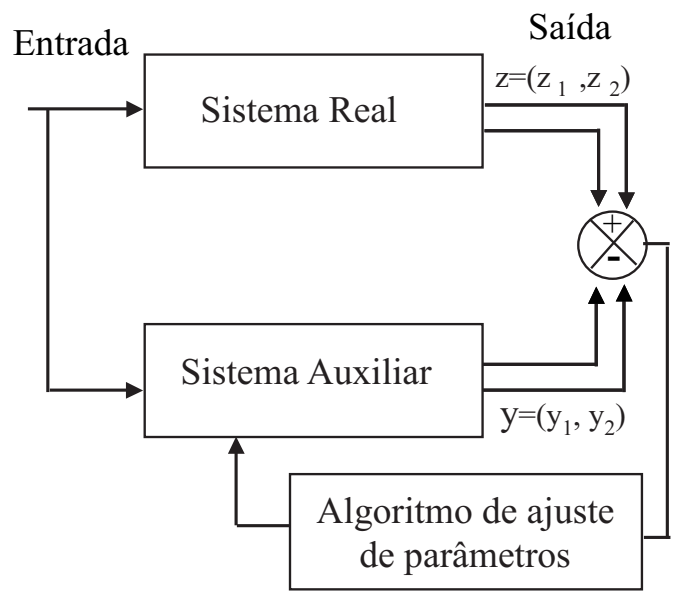

(a)

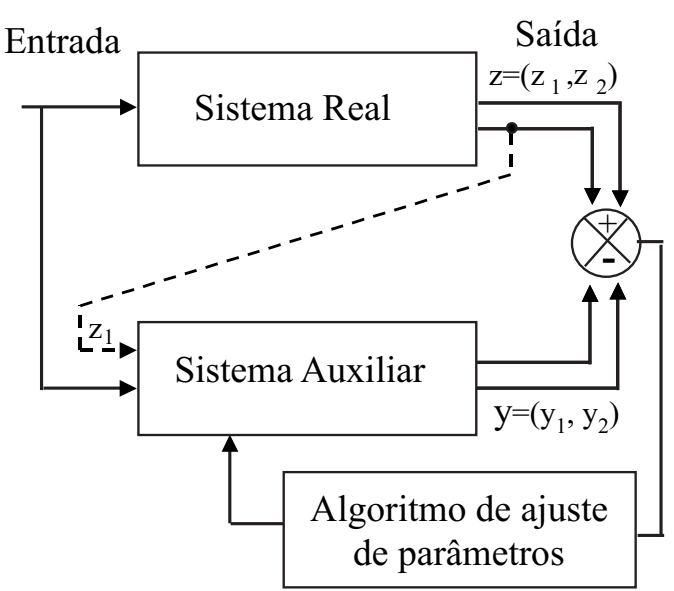

(b)

Figura 3.2: Estimação de parâmetros. (a) com a abordagem original, (b) com o acoplamento mestre-escravo.

O acoplamento muda as equações diferenciais do sistema auxiliar e as equações de sensibilidade. Não existe um procedimento sistemático para a escolha das variáveis que farão parte do acoplamento mestre-escravo. Neste trabalho, a escolha destas variáveis é realizada a fim de tornar mais simples as equações do sistema auxiliar. Para isto, substitui-se as variáveis de acoplamento nos termos não-lineares do sistema auxiliar. A experiência adquirida fazendo isto tem mostrado que a região de convergência da metodologia aumenta após está substituição. Entretanto, investigações mais detalhadas deste assunto são necessárias para um completo entendimento deste problema. Como ilustração, mostra-se a influência das entradas de sincronização no sistema massa-mola estudado no Capítulo 2 (Exemplo 2.3).

Exemplo 3.1 No sistema massa-mola, estuda-se a influência da entrada de sincronização $z_{1}$. As equações do sistema auxiliar (2.2) após a substituição direta 
da entrada $z_{1}$ fica da seguinte forma,

$$
\begin{aligned}
\dot{x}_{1} & =x_{2} \\
\dot{x}_{2} & =\frac{u(t)}{m}-\frac{k}{m} z_{1} \\
y & =x_{1}
\end{aligned}
$$

neste caso, o valor medido da posição do sistema $z_{1}$ é utilizado no lugar da variável $x_{1}$ no sistema (3.3).

As equações diferenciais das funções de sensibilidade em relação ao parâmetro $k$ após a inclusão da entrada $z_{1}$ ficam,

$$
\begin{aligned}
\dot{\lambda}_{x_{1}}^{k} & =\lambda_{x_{2}}^{k}, \\
\dot{\lambda}_{x_{2}}^{k} & =-\frac{1}{m} z_{1}, \\
\lambda_{y}^{k} & =\lambda_{x_{1}}^{k},
\end{aligned}
$$

sendo $\lambda_{x_{1}}^{k}=\frac{\partial x_{1}}{\partial k}, \lambda_{x_{2}}^{k}=\frac{\partial x_{2}}{\partial k}$ e $\lambda_{y}^{k}=\frac{\partial y}{\partial k}$

As equações diferenciais das funções de sensibilidade em relação ao parâmetro $m$ após a inclusão do entrada $z_{1}$ ficam,

$$
\begin{aligned}
\dot{\lambda}_{x_{1}}^{m} & =\lambda_{x_{2}}^{m}, \\
\dot{\lambda}_{x_{2}}^{m} & =-\frac{1}{m^{2}} u(t)+\frac{k}{m^{2}} z_{1}, \\
\lambda_{y}^{m} & =\lambda_{x_{1}}^{m}
\end{aligned}
$$

sendo $\lambda_{x_{1}}^{m}=\frac{\partial x_{1}}{\partial m}, \lambda_{x_{2}}^{m}=\frac{\partial x_{2}}{\partial m}$ e $\lambda_{y}^{m}=\frac{\partial y}{\partial m}$.

Comparando o sistema auxiliar (2.2), as equações de sensibilidade (2.11)(2.12) sem acoplamento, com os mesmos após o acoplamento (3.3), (3.4)-(3.5), observa-se que alguns termos no sistema de equações foram simplificados. Por exemplo, a equação de sensibilidade de $x_{2}$ em relação a $m$ com o acoplamento (Equação 3.5) possui dois termos e depende dos parâmetros, da entrada $u(t)$ e da entrada de acoplamento $z_{1}$ (a qual é conhecida); enquanto que a mesma sem acoplamento (Equação 2.12) possui três termos e depende dos parâmetros, da entrada $u(t)$ e da variável de estado $x_{1}$ (a qual não é conhecida). A inclusão do acoplamento simplificou as equações de sensibilidade e em geral resulta no 
aumento a região de convergência do algoritmo de ajuste. As funções de sensibilidade com o uso de acoplamento mestre-escravo são mostradas na Figura 3.3.

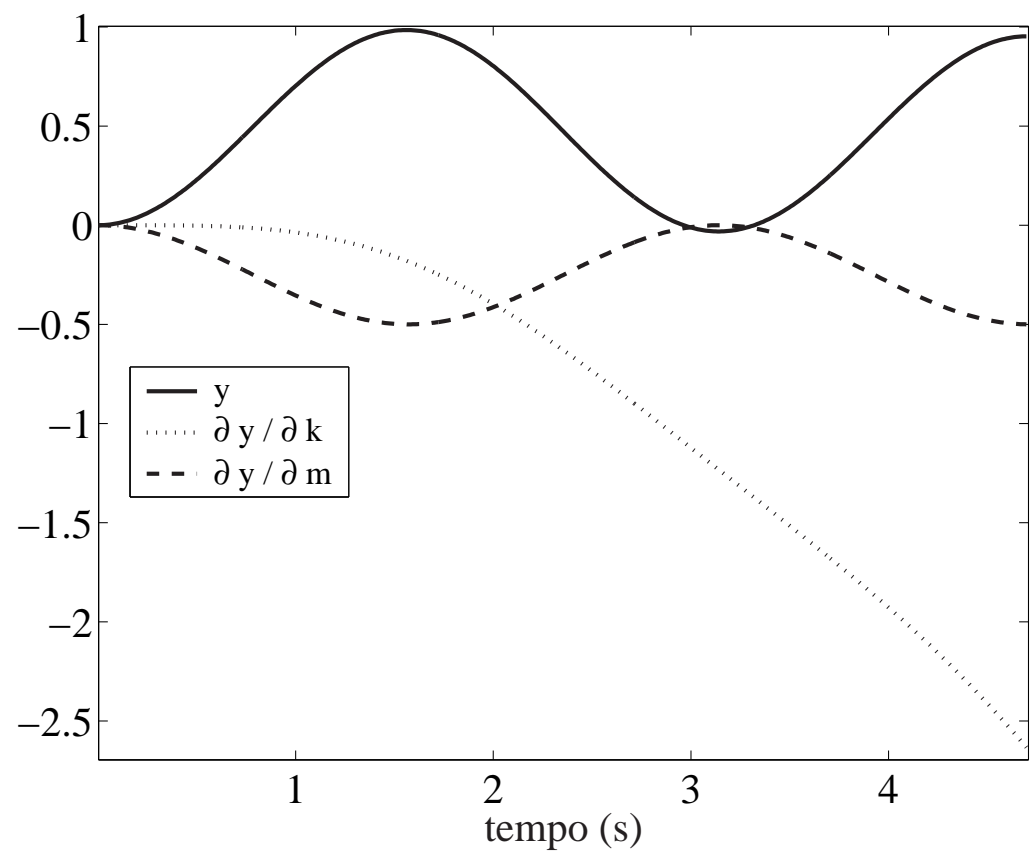

Figura 3.3: Gráfico da saída e funções de sensibilidade para o Exemplo 3.1

Comparando as funções de sensibilidade com e sem acoplamento, (Figuras 2.4 e 3.3, respectivamente) pode observa-se que para o caso sem acoplamento o comportamento é oscilatório e as amplitudes de $\frac{\partial y}{\partial k} \mathrm{e} \frac{\partial y}{\partial m}$ são proporcionais. Entretanto, após o acoplamento, as funções de sensibilidade possuem comportamentos diferentes. Enquanto $\frac{\partial y}{\partial m}$ continua sendo oscilatória, a função de sensibilidade em relação ao parâmetro $\frac{\partial y}{\partial k}$ torna-se monotônica. Assim, o parâmetro $k$ será responsável por "acertar" o comportamento de regime permanente, enquanto $m$ "acerta" a freqüência de oscilação no regime transitório. Isto permite que os parâmetros do sistema massa-mola sejam estimados com maior facilidade para o caso com acoplamento.

Nos próximos capítulos, o acoplamento mestre-escravo será utilizado na metodologia de estimação de parâmetros de sistemas não-lineares com o objetivo de aumentar a região de convergência. 


\section{Capítulo 4}

\section{Estimação de Parâmetros de Sistemas Dinâmicos Não-Lineares}

Este capítulo apresenta a aplicação da metodologia de estimação de parâmetros, previamente apresentada em sistemas dinâmicos não-lineares. Também são mostradas as vantagens do uso do acoplamento mestre-escravo para aumentar a região de convergência quando comparada com a metodologia tradicional de sensibilidade de trajetória sem acoplamento.

\subsection{Estimação de Parâmetros do Sistema Massa- Mola}

Nesta seção, estimam-se os parâmetros do sistema massa mola do exemplo 2.1 evidenciando os benefícios do acoplamento mestre-escravo no processo de estimação. Nas Figuras 4.1 e 4.2, comparam-se os diagramas de blocos da aplicação da metodologia de sensibilidade de trajetória sem acoplamento mestre-escravo (forma tradicional) e com o acoplamento mestre-escravo (proposta). As equações do sistema auxiliar e as equações de sensibilidade para a formulação tradicional foram obtidas de $(2.2)$ e $(2.11-2.12)$, respectivamente; e considerando $z_{1}(t)$ como variável de acoplamento, estas equações são (3.3) e (3.4)-(3.5), respectivamente. Os dados do sistema real foram obtidos via simulação, usando como 
valores verdadeiros dos parâmetros $k_{r}=8$ e $m_{r}=2$, e condições iniciais $z_{1}(0)=0$ e $z_{2}(0)=0$.

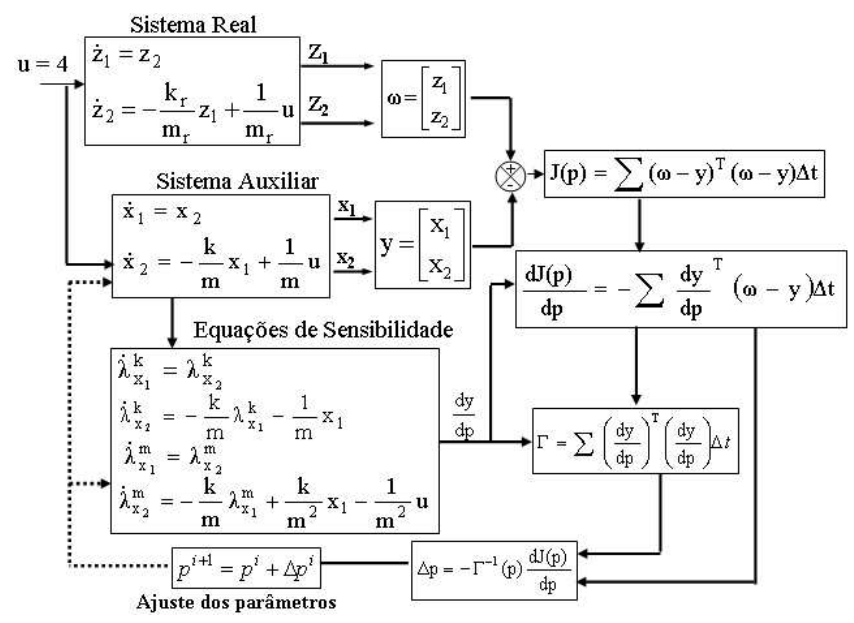

Figura 4.1: Metodologia de sensibilidade de trajetória na formulação tradicional.

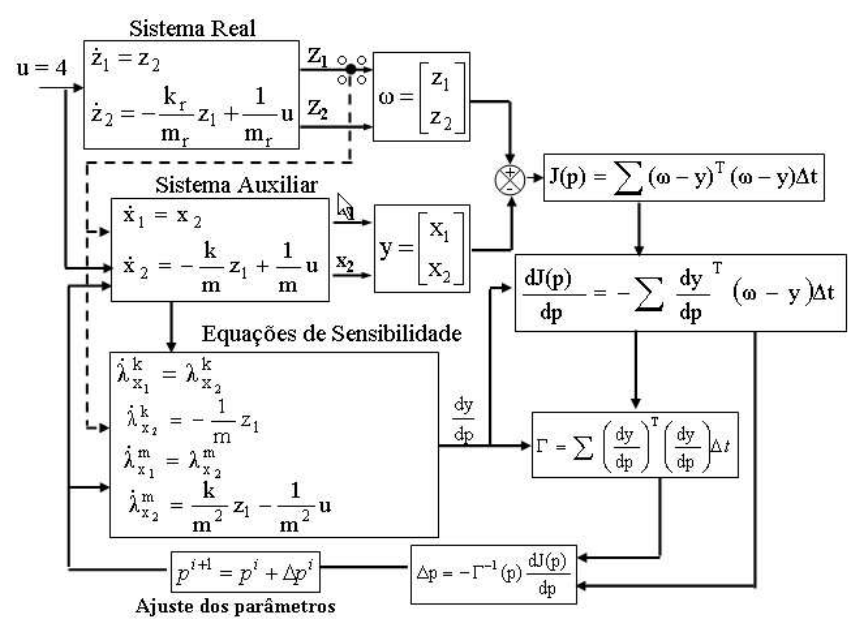

Figura 4.2: Metodologia de sensibilidade de trajetória usando o acoplamento mestreescravo.

Para avaliar o efeito do acoplamento mestre-escravo na metodologia de sensibilidade de trajetória comparou-se a máxima incerteza nos valores iniciais dos parâmetros para a qual a metodologia conseguia estimar os parâmetros antes e depois da inclusão do acoplamento.

Para o caso da metodologia de sensibilidade de trajetória na formulação tradicional, a metodologia consegue estimar os parâmetros mesmo com alterações 
entre $-37 \%$ e $+37 \%$ em relação aos valores verdadeiros. Já para o caso que utiliza a metodologia de sensibilidade de trajetória com acoplamento mestre-escravo, a metodologia consegue estimar os parâmetros mesmo com alterações entre $-98 \%$ e $+98 \%$ em relação aos valores verdadeiros. Estes valores máximos de alterações foram obtidos aumentando a incerteza nos parâmetros gradativamente e verificando se a metodologia convergia a valores próximos dos verdadeiros.

Nas Tabela 4.1 mostra-se, como ilustração, a convergência a valores errados após 38 iterações com a metodologia de sensibilidade de trajetória sem acoplamento mestre-escravo no caso em que os valores iniciais de $k$ e $m$ estão distantes em $-50 \%$ e $+50 \%$ dos seus valores reais. O mesmo caso, mas com a inclusão do acoplamento mestre-escravo na metodologia de sensibilidade de trajetória é mostrado na Tabela 4.2. Como é observado a convergência aos valores verdadeiros é garantida neste caso após 5 iterações.

Tabela 4.1: Resultados com a metodologia de sensibilidade de trajetória (formulação tradicional).

\begin{tabular}{|c|c|c|c|}
\hline Iter. & $\mathrm{k}$ & $\mathrm{m}$ & $\begin{array}{c}J p \\
(\%)\end{array}$ \\
\hline 0 (inicial) & 4 & 3 & 2,4385 \\
\hline 1 & 6,7802 & 5,1360 & 0,8181 \\
\hline 2 & 10,0631 & 7,7746 & 0,5268 \\
\hline 3 & 12,4527 & 9,9772 & 0,4928 \\
\hline 4 & 13,2303 & 11,1923 & 0,4896 \\
\hline 5 & 13,3847 & 12,0302 & 0,4875 \\
\hline 6 & 13.4747 & 12,8280 & 0,4870 \\
\hline$\vdots$ & $\vdots$ & $\vdots$ & $\vdots$ \\
\hline 38 (final) & 13,6321 & 16,1144 & 0,4830 \\
\hline verdadeiro & 8,000 & 2,000 & - \\
\hline
\end{tabular}


Tabela 4.2: Resultados com a metodologia de sensibilidade de trajetória com acoplamento mestre-escravo.

\begin{tabular}{|c|c|c|c|}
\hline Iter. & $\mathrm{k}$ & $\mathrm{m}$ & $\begin{array}{c}J p \\
(\%)\end{array}$ \\
\hline 0 (inicial) & 4 & 3 & 22,01 \\
\hline 1 & 10,006 & 1,492 & 22,71 \\
\hline 2 & 8,485 & 1,861 & 0,874 \\
\hline 3 & 8,021 & 1,980 & 0,0029 \\
\hline 4 & 7,991 & 1,989 & 0,0001 \\
\hline 5 (final) & 7,991 & 1,989 & 0,0001 \\
\hline verdadeiro & 8,000 & 2,000 & - \\
\hline
\end{tabular}

\subsection{Estimação de Parâmetros do Sistema de Lo- renz}

Esta seção apresenta a estimação dos parâmetros do sistema caótico de Lorenz. Trajetórias de sistemas caóticos possuem alta sensibilidade em relação as condições iniciais e aos parâmetros. Esta característica, por um lado, é positiva no sentido em que a saída sempre contém informações significativas dos parâmetros, mas por outro lado, pode acarretar problemas numéricos, especialmente quando a sensibilidade relativa entre os parâmetros for grande.

Entre as abordagens propostas na literatura para estimação de parâmetros de sistemas caóticos, o controle adaptativo baseado em sincronização tem sido bastante utilizado (Parlitz, 2006; Huang e Guo, 2004; Huang, 2006). Em (Huang e Guo, 2004; Rahul, 2003; Yu et al., 2007) propõe-se um método baseado em sincronização para estimar parâmetros de sistemas caóticos utilizando uma lei de controle não-linear (projetada por função de Lyapunov). Uma abordagem similar que utiliza sincronização e controle geométrico é apresentada em (Freitas et al., 2005). Outras abordagens que utilizam sincronização e outras ferramentas auxiliares podem ser encontradas em (Ines e Joaquin, 2005; Yechiel, 2002). 
Neste trabalho, a metodologia de sensibilidade de trajetória é utilizada para estimar os parâmetros deste sistema. As vantagens da metodologia de sensibilidade de trajetória em relação às metodologias que dependem de uma lei de controle para o ajuste de parâmetros são:

a. Pode ser facilmente implementada para qualquer sistema não-linear (não depende da existência de uma função de Lyapunov, por exemplo);

b. Requer um intervalo de aferição de medidas pequeno, pois o processo é realizado de forma iterativa em comparação com o método baseado em controle adaptativo, que precisa que as medidas sejam obtidas em um intervalo de tempo maior para que a sincronização das saídas seja atingida;

c. Pode ser utilizado para estimar as condições iniciais das variáveis de estados do sistema o que não é possível com o método baseado em controle adaptativo.

Entretanto, a metodologia de sensibilidade de trajetória, como qualquer método iterativo de estimação de parâmetros não pode garantir que os parâmetros serão estimados corretamente para qualquer valor inicial dos parâmetros. O sucesso da estimação depende do valor dos parâmetros utilizados no início do processo. Além disso, apresenta problemas numéricos quando as sensibilidades relativas do vetor de saída em relação a cada parâmetro forem muito diferentes, como é o caso do sistema caótico de Lorenz.

Algumas alternativas são apresentadas na seqüência com o objetivo de contornar estas dificuldades.

Considere o sistema de Lorenz

$$
\begin{aligned}
& \dot{x}_{1}=-\sigma_{r} x_{1}+\sigma_{r} x_{2}, \\
& \dot{x}_{2}=-x_{2}-x_{1} x_{3}+r_{r} x_{1}, \\
& \dot{x}_{3}=-b_{r} x_{3}+x_{1} x_{2},
\end{aligned}
$$

em que $x=\left(x_{1}, x_{2}, x_{3}\right)^{T}$ é o vetor de variáveis de estados, $\sigma_{r}=10, r_{r}=20 \mathrm{e}$ $b_{r}=2,66$ são os valores verdadeiros dos parâmetros e as condições iniciais são 
$\left(x_{1 o}, x_{2 o}, x_{3 o}\right)=(2,3,7)$. Considera-se que $x_{1}$ e $x_{2}$ são disponíveis do conjunto de medidas.

Dependendo da escolha das entradas e das saídas, foram testadas as seguintes alternativas: a) usando a metodologia de sensibilidade de trajetória tradicional, b) usando a metodologia de sensibilidade de trajetória com acoplamento mestreescravo e c) mudando as entradas e as saídas.

\subsubsection{Metodologia de sensibilidade de trajetória na for- mulação tradicional}

Nesta alternativa, o sistema auxiliar é modelado por

$$
\begin{aligned}
& \dot{z}_{1}=-\sigma z_{1}+\sigma z_{2}, \\
& \dot{z}_{2}=-z_{2}-z_{1} z_{3}+r z_{1}, \\
& \dot{z}_{3}=-b z_{3}+z_{1} z_{2} .
\end{aligned}
$$

Este sistema possui a mesma estrutura do sistema real, a única diferença é que as variáveis de estado $x_{i}, i=1,2,3$ foram substituídas por $z_{i}, i=1,2,3$ e os parâmetros não possuem o sub-índice $r$. O vetor de saída é $y=\left(z_{1}, z_{2}\right)$. Como $x_{1}$ e $x_{2}$ são disponíveis do conjunto de medidas, utiliza-se $z_{1 o}=x_{1 o}$ e $z_{2 o}=x_{2 o}$. Por outro lado $z_{3 o}$ não é conhecido e deve ser estimado. O vetor de parâmetros fica então: $p=\left(\sigma, r, b, z_{3 o}\right)$. Admite-se, neste primeiro teste, que não existe erro na medida.

As equações de sensibilidade são obtidas derivando-se o sistema auxiliar em relação a cada parâmetro $p_{j}$. Para isso, faz-se a seguinte substituição $\lambda_{z_{i}}^{p_{j}}=\frac{\partial z_{i}}{p_{j}}$, $i=1, \ldots, 3 ; j=1, \ldots, 4$.

Em relação $\sigma$ obtém-se:

$$
\begin{aligned}
& \dot{\lambda}_{z_{1}}^{\sigma}=-z_{1}+z_{2}+\sigma\left(\lambda_{z_{2}}^{\sigma}-\lambda_{z_{1}}^{\sigma}\right), \\
& \dot{\lambda}_{z_{2}}^{\sigma}=-\lambda_{z_{2}}^{\sigma}-z_{3} \lambda_{z_{1}}^{\sigma}-z_{1} \lambda_{z_{3}}^{\sigma}+r \lambda_{z_{1}}^{\sigma}, \\
& \dot{\lambda}_{z_{3}}^{\sigma}=-b \lambda_{z_{3}}^{\sigma}+z_{1} \lambda_{z_{2}}^{\sigma}+z_{2} \lambda_{z_{1}}^{\sigma},
\end{aligned}
$$

com condições iniciais em $t=t_{o}: \lambda_{z_{1}}^{\sigma}\left(t_{o}\right)=\lambda_{z_{2}}^{\sigma}\left(t_{o}\right)=\lambda_{z_{3}}^{\sigma}\left(t_{o}\right)=0$. 
Em relação a $r$ :

$$
\begin{aligned}
& \dot{\lambda}_{z_{1}}^{r}=\sigma\left(\lambda_{z_{2}}^{r}-\lambda_{z_{1}}^{r}\right) \\
& \dot{\lambda}_{z_{2}}^{r}=-\lambda_{z_{2}}^{r}-z_{1} \lambda_{z_{3}}^{r}-z_{3} \lambda_{z_{1}}^{r}+z_{1}+r \lambda_{z_{1}}^{r}, \\
& \dot{\lambda}_{z_{3}}^{r}=-b \lambda_{z_{3}}^{r}+z_{1} \lambda_{z_{2}}^{r}+z_{2} \lambda_{z_{1}}^{r}
\end{aligned}
$$

com condições iniciais em $t=t_{o}: \lambda_{z_{1}}^{r}\left(t_{o}\right)=\lambda_{z_{2}}^{r}\left(t_{o}\right)=\lambda_{z_{3}}^{r}\left(t_{o}\right)=0$.

Em relação a $b$ :

$$
\begin{aligned}
& \dot{\lambda}_{z_{1}}^{b}=\sigma\left(\lambda_{z_{2}}^{b}-\lambda_{z_{1}}^{b}\right) \\
& \dot{\lambda}_{z_{2}}^{b}=-\lambda_{z_{2}}^{b}-z_{1} \lambda_{z_{3}}^{b}-z_{3} \lambda_{z_{1}}^{b}+r \lambda_{z_{1}}^{b}, \\
& \dot{\lambda}_{z_{3}}^{b}=-z_{3}-b \lambda_{z_{3}}^{b}+z_{1} \lambda_{z_{2}}^{b}+z_{2} \lambda_{z_{1}}^{b}
\end{aligned}
$$

com condições iniciais em $t=t_{o}: \lambda_{z_{1}}^{b}\left(t_{o}\right)=\lambda_{z_{2}}^{b}\left(t_{o}\right)=\lambda_{z_{3}}^{b}\left(t_{o}\right)=0$.

Em relação ao parâmetro $z_{30}$ :

$$
\begin{aligned}
\dot{\lambda}_{z_{1}}^{z_{3 o}} & =\sigma\left(\lambda_{z_{2}}^{z_{3 o}}-\lambda_{z_{1}}^{z_{3 o}}\right) \\
\dot{\lambda}_{z_{2}}^{z_{3 o}} & =-\lambda_{z_{2}}^{z_{3 o}}-z_{1} \lambda_{z_{3}}^{z_{3 o}}-z_{3} \lambda_{z_{1}}^{z_{3 o}}+r \lambda_{z_{1}}^{z_{3 o}}, \\
\dot{\lambda}_{z_{3}}^{z_{3 o}} & =-b \lambda_{z_{3}}^{z_{3 o}}+z_{1} \lambda_{z_{2}}^{z_{3 o}}+z_{2} \lambda_{z_{1}}^{z_{3 o}},
\end{aligned}
$$

com condições iniciais em $t=t_{o}: \lambda_{z_{1}}^{z_{3 o}}\left(t_{o}\right)=\lambda_{z_{1}}^{z_{3 o}}\left(t_{o}\right)=0 ; \lambda_{z_{3}}^{z_{3 o}}\left(t_{o}\right)=1$.

As funções de sensibilidade de trajetória dos estados são obtidas resolvendo-se os sistemas de equações de sensibilidade (4.3)-(4.6). As funções de sensibilidade do vetor de saída são obtidas pela substituição das sensibilidades dos estados. Por exemplo, a sensibilidade da saída em relação ao parâmetro $\sigma$ é: $\lambda_{y}^{\sigma}=\left(\lambda_{z_{1}}^{\sigma}, \lambda_{z_{2}}^{\sigma}\right)^{T}$.

A partir da solução do sistema auxiliar (4.2) e das funções de sensibilidade, $\frac{\partial J(p)}{\partial p}$ e $\Gamma$ podem ser determinadas usando (2.25) e (2.27), respectivamente. Na Figura 4.3, mostra-se o diagrama de blocos com esta alternativa.

Os resultados mostram que usando a metodologia de sensibilidade de trajetória tradicional (sem acoplamento mestre-escravo), a convergência só é garantida se o valor inicial do vetor de parâmetros estiver muito próximo do valor verdadeiro (no máximo $\pm 0.5 \%$ distante). Para incertezas no vetor de parâmetros superior a $\pm 0,5 \%$ a metodologia não converge. 


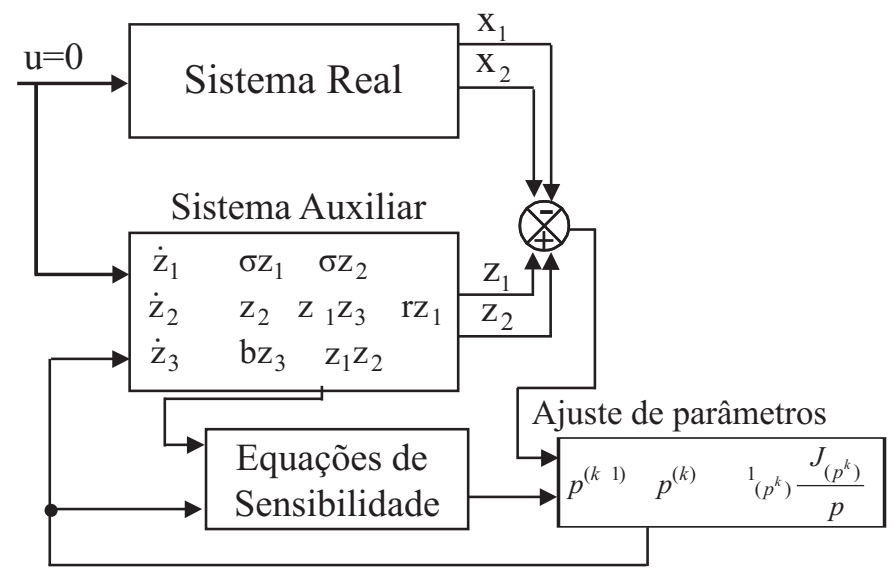

Figura 4.3: Estimação de parâmetros do sistema de Lorenz usando a metodologia de sensibilidade de trajetória tradicional.

A Figura 4.4 mostra uma comparação entre a saída \#1 do sistema real e auxiliar no início do processo de estimação, considerando o valor inicial dos parâmetros do sistema auxiliar em $+2 \%$ do valor correto. As saídas do sistema real e do sistema auxiliar são muito diferentes, mesmo com esta pequena diferença de parâmetros. Além disso, na Figura 4.5, mostram-se as funções de sensibilidade de trajetória de $z_{1}$ em relação aos parâmetros. Observa-se que $\frac{\partial z_{1}}{\partial \sigma}$ é muito pequena quando comparada com as outras funções de sensibilidade. Está última característica é um dos principais motivos que dificulta a estimação simultânea de todos parâmetros.

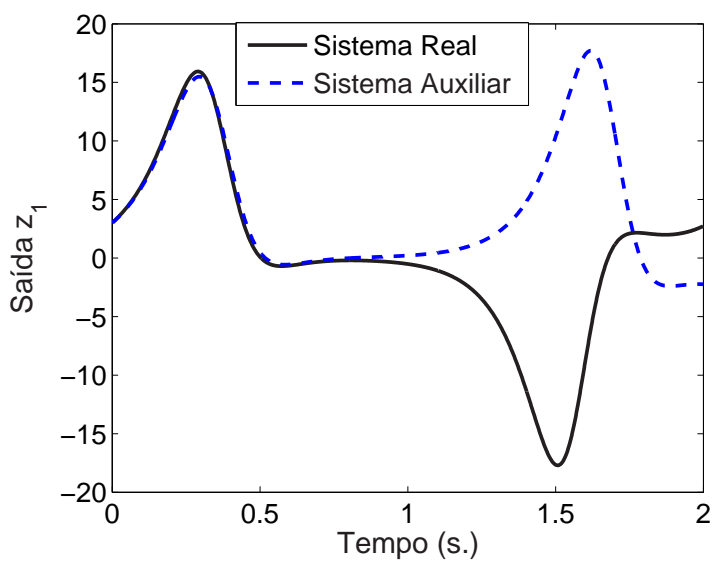

Figura 4.4: Saídas do sistema real e auxiliar considerando que os parâmetros do sistema auxiliar estão $+2 \%$ distantes dos valores corretos. 


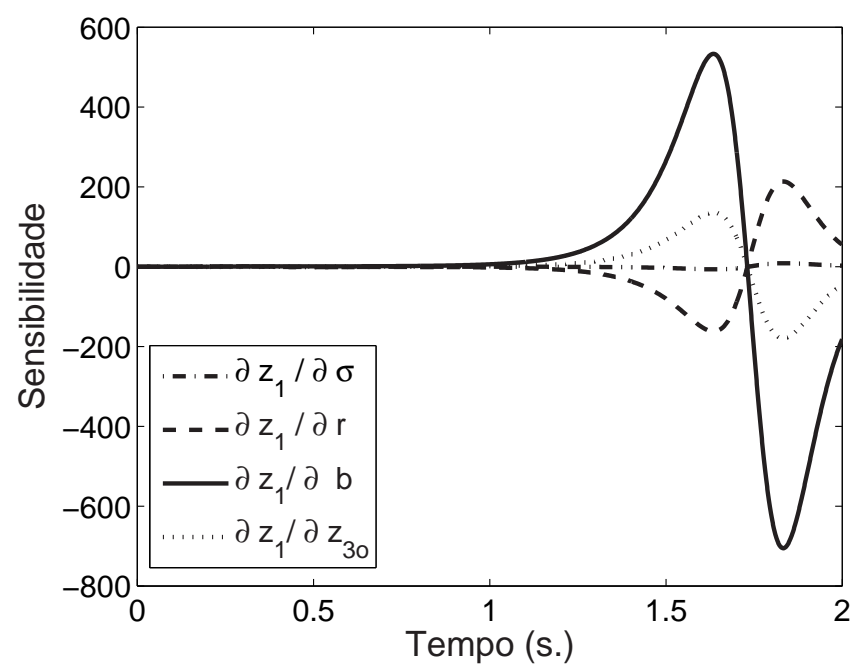

Figura 4.5: Funções de sensibilidade de trajetória da variável $z_{1}$ em relação aos parâmetros.

Na seqüência, duas abordagens são propostas com o objetivo de aumentar a região de convergência.

\subsubsection{Metodologia de sensibilidade de trajetória com aco- plamento mestre-escravo.}

Nesta seção, o acoplamento mestre-escravo é usado para melhorar a robustez numérica do algoritmo de estimação baseado em sensibilidade de trajetória. O sistema auxiliar é representado por (4.2) mudando apenas a variável $z_{1}$ pela entrada de acoplamento $x_{1}$ em alguns termos. A experiência da aplicação desta técnica, mostra que a região de convergência aumenta após a inclusão do acoplamento, principalmente quando o acoplamento é feito em termos não-lineares.

$$
\begin{aligned}
& \dot{z}_{1}=\sigma z_{2}-\sigma z_{1} \\
& \dot{z}_{2}=-z_{2}-x_{1} z_{3}+r x_{1} \\
& \dot{z}_{3}=x_{1} z_{2}-b z_{3} .
\end{aligned}
$$

O vetor de saída é $y=\left(z_{1}, z_{2}\right)$ e o vetor de parâmetros é $p=\left(\sigma, r, b, z_{o}\right)$. As equações de sensibilidade das variáveis de estado são obtidas derivando-se (4.7) 
em relação a cada parâmetro, para isso faz-se a seguinte substituição $\lambda_{z_{i}}^{p_{j}}=\frac{\partial z_{i}}{p_{j}}$, $i=1, \ldots, 3 ; j=1, \ldots, 4$.

Em relação a $\sigma$ obtém-se:

$$
\begin{aligned}
& \dot{\lambda}_{z_{1}}^{\sigma}=z_{2}-z_{1}+\sigma\left(\lambda_{z_{2}}^{\sigma}-\lambda_{z_{1}}^{\sigma}\right), \\
& \dot{\lambda}_{z_{2}}^{\sigma}=-\lambda_{z_{2}}^{\sigma}-x_{1} \lambda_{z_{3}}^{\sigma}, \\
& \dot{\lambda}_{z_{3}}^{\sigma}=x_{1} \lambda_{z_{2}}^{\sigma}-b \lambda_{z_{3}}^{\sigma},
\end{aligned}
$$

com condições iniciais em $t=t_{o}: \lambda_{z_{1}}^{\sigma}\left(t_{o}\right)=\lambda_{z_{2}}^{\sigma}\left(t_{o}\right)=\lambda_{z_{3}}^{\sigma}\left(t_{o}\right)=0$.

Em relação a $r$ :

$$
\begin{aligned}
& \dot{\lambda}_{z_{1}}^{r}=\sigma\left(\lambda_{z_{2}}^{r}-\lambda_{z_{1}}^{r}\right), \\
& \dot{\lambda}_{z_{2}}^{r}=-\lambda_{z_{2}}^{r}-x_{1} \lambda_{z_{3}}^{r}+x_{1}, \\
& \dot{\lambda}_{z_{3}}^{r}=x_{1} \lambda_{z_{2}}^{r}-b \lambda_{z_{3}}^{r},
\end{aligned}
$$

com condições iniciais em $t=t_{o}: \lambda_{z_{1}}^{r}\left(t_{o}\right)=\lambda_{z_{2}}^{r}\left(t_{o}\right)=\lambda_{z_{3}}^{r}\left(t_{o}\right)=0$.

Em relação a $b$ :

$$
\begin{aligned}
& \dot{\lambda}_{z_{1}}^{b}=\sigma\left(\lambda_{z_{2}}^{b}-\lambda_{z_{1}}^{b}\right), \\
& \dot{\lambda}_{z_{2}}^{b}=-\lambda_{z_{2}}^{b}-x_{1} \lambda_{z_{3}}^{b}, \\
& \dot{\lambda}_{z_{3}}^{b}=x_{1} \lambda_{z_{2}}^{b}-b \lambda_{z_{3}}^{b}-z_{3},
\end{aligned}
$$

com condições iniciais em $t=t_{o}: \lambda_{z_{1}}^{b}\left(t_{o}\right)=\lambda_{z_{2}}^{b}\left(t_{o}\right)=\lambda_{z_{3}}^{b}\left(t_{o}\right)=0$.

Em relação a $z_{3 o}$ :

$$
\begin{aligned}
& \dot{\lambda}_{z_{1}}^{z_{3 o}}=\sigma\left(\lambda_{z_{2}}^{z_{3 o}}-\lambda_{z_{1}}^{z_{3 o}}\right), \\
& \dot{\lambda}_{z_{2}}^{z_{3 o}}=-\lambda_{z_{2}}^{z_{3 o}}-x_{1} \lambda_{z_{3}}^{z_{3 o}}, \\
& \dot{\lambda}_{z_{3}}^{z_{3 o}}=x_{1} \lambda_{z_{2}}^{z_{3 o}}-b \lambda_{z_{3}}^{z_{3 o}} .
\end{aligned}
$$

com condições iniciais em $t=t_{o}: \lambda_{z_{1}}^{z_{3 o}}\left(t_{o}\right)=\lambda_{z_{2}}^{z_{3 o}}\left(t_{o}\right)=\lambda_{z_{3}}^{z_{3 o}}\left(t_{o}\right)=0$.

As funções de sensibilidade de trajetória dos estados são obtidas integrando-se as equações de sensibilidade (4.8)-(4.11). As funções de sensibilidade do vetor de saída são obtidas pela substituição das sensibilidades dos estados. Por exemplo, a sensibilidade da saída em relação ao parâmetro $\sigma$ é: $\lambda_{y}^{\sigma}=\left(\lambda_{z_{1}}^{\sigma}, \lambda_{z_{2}}^{\sigma}\right)^{T}$. 
A Figura 4.6 mostra o diagrama esquemático da aplicação da metodologia. O bloco "Equações de Sensibilidade" está formado por (4.8)-(4.11). O bloco "Ajuste de Parâmetros" atualiza os parâmetros em cada iteração "k". A matriz $\Gamma$ e o vetor $\frac{\partial J(p)}{\partial p}$ podem ser determinados a partir da solução do sistema auxiliar (4.7) e das equações de sensibilidade (4.8)-(4.11) usando as (2.25) e (2.27), respectivamente, seguindo o procedimento da Seção 2.6.

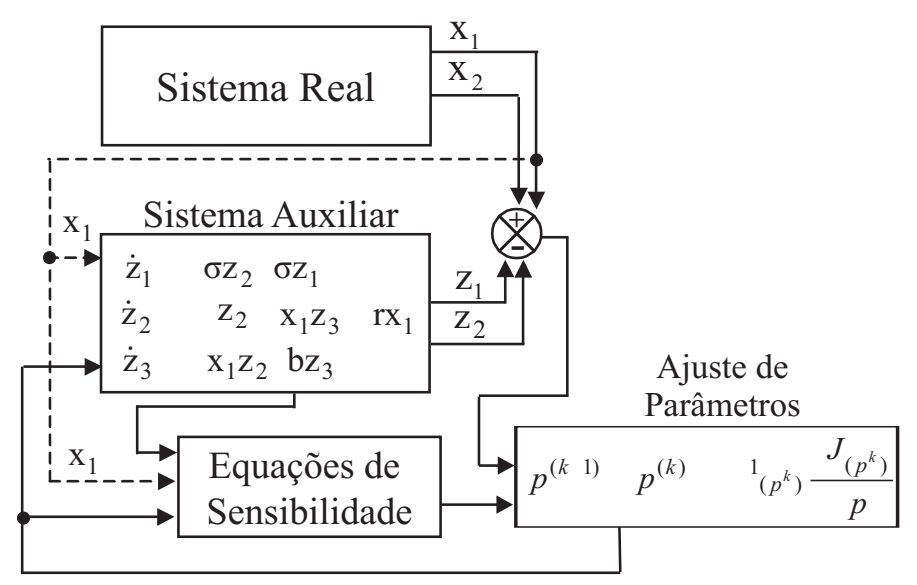

Figura 4.6: Estimação de parâmetros do sistema de Lorenz baseada em sensibilidade de trajetória e acoplamento mestre-escravo.

Analisando o sistema auxiliar e as equações de sensibilidade, nota-se que alguns termos ou variáveis foram eliminados e outros acrescentados com relação às equações da alternativa da Seção 4.2 .1 (Equações (4.2) e (4.3)-(4.6), respectivamente). Isto permite que os parâmetros convirjam aos valores verdadeiros mesmo quando a estimativa inicial dos parâmetros estiver distante dos valores verdadeiros.

Os resultados mostram que a metodologia consegue estimar os parâmetros corretos mesmo com alterações entre $-60 \%$ e $+60 \%$ em relação aos valores verdadeiros. Na tabela 4.3 são apresentados os resultados da estimação dos parâmetros de um dos testes.

\section{Influência do ruído nas medidas.}

A fim de avaliar o desempenho da metodologia de sensibilidade de trajetória com acoplamento mestre escravo na presença de ruído, o teste anterior foi repetido 
Tabela 4.3: Estimação dos parâmetros usando o acoplamento mestre-escravo.

\begin{tabular}{|c|c|c|c|c|c|}
\hline parâmetro & $\begin{array}{c}\text { Valor } \\
\text { inicial }\end{array}$ & $\begin{array}{c}\text { Desvio } \\
(\%)\end{array}$ & $\begin{array}{c}\text { Valor } \\
\text { final }\end{array}$ & $\begin{array}{c}\text { Valor } \\
\text { real }\end{array}$ & $\begin{array}{c}\text { Erro } \\
(\%)\end{array}$ \\
\hline$\sigma$ & 4,0 & $-60 \%$ & 10 & 10 & 0 \\
\hline$r$ & 8,0 & $-60 \%$ & 20 & 20 & 0 \\
\hline$b$ & 4,2 & $+60 \%$ & 2,66 & 2.66 & 0 \\
\hline$z_{3 o}$ & 2,8 & $-60 \%$ & 6,99 & 7,00 & $-0,1$ \\
\hline
\end{tabular}

considerando ruído branco Gaussiano de média zero e desvio padrão 5\% em todas as medidas. Com isto, além de estimar $\sigma, r, b$ e $z_{3 o}$, serão estimadas as outras condições iniciais $\left(z_{1 o}\right.$ e $\left.z_{2 o}\right)$. O vetor de parâmetros torna-se $p=\left(\sigma, r, b, z_{1 o}, z_{2 o}\right.$, $\left.z_{30}\right)$.

As equações de sensibilidade em relação aos dois novos parâmetros são:

Em relação a $z_{10}$ :

$$
\begin{aligned}
& \dot{\lambda}_{z_{1}}^{z_{1 o}}=\sigma\left(\lambda_{z_{2}}^{z_{1 o}}-\lambda_{z_{1}}^{z_{1 o}}\right), \\
& \dot{\lambda}_{z_{2}}^{z_{1 o}}=-\lambda_{z_{2}}^{z_{1 o}}-x_{1} \lambda_{z_{3}}^{z_{1 o}}, \\
& \dot{\lambda}_{z_{3}}^{z_{1 o}}=x_{1} \lambda_{z_{2}}^{z_{1 o}}-b \lambda_{z_{3}}^{z_{1 o}},
\end{aligned}
$$

com condições iniciais em $t=t_{o}: \lambda_{z_{1}}^{z_{1 o}}\left(t_{o}\right)=1 ; \lambda_{z_{2}}^{z_{1 o}}=\lambda_{z_{3}}^{z_{1 o}}=0$.

Em relação a $z_{2 o}$ :

$$
\begin{aligned}
& \dot{\lambda}_{z_{1}}^{z_{2 o}}=\sigma\left(\lambda_{z_{2}}^{z_{2 o}}-\lambda_{z_{1}}^{z_{2 o}}\right), \\
& \dot{\lambda}_{z_{2}}^{z_{2 o}}=-\lambda_{z_{2}}^{z_{2 o}}-x_{1} \lambda_{z_{3}}^{z_{2 o}}, \\
& \dot{\lambda}_{z_{3}}^{z_{2 o}}=x_{1} \lambda_{z_{2}}^{z_{2 o}}-b \lambda_{z_{3}}^{z_{2 o}},
\end{aligned}
$$

com condições iniciais em $t=t_{o}: \lambda_{z_{1}}^{z_{2 o}}\left(t_{o}\right)=0 ; \lambda_{z_{2}}^{z_{2 o}}\left(t_{o}\right)=1 ; \lambda_{z_{3}}^{z_{2 o}}\left(t_{o}\right)=0$.

Os parâmetros foram corretamente estimados até nos casos em que os valores iniciais dos parâmetros estavam distanciados em $\pm 54 \%$ em relação aos valores verdadeiros. Os valores iniciais considerados para $z_{1 o}$ e $z_{2 o}$ foram os valores iniciais $x_{1 o}$ e $x_{2 o}$ (os quais possuem incerteza de $5 \%$ ocasionado pelo ruído).

As saídas do sistema real e do sistema auxiliar no começo e no final das iterações são mostradas na Figura 4.7. 
Tabela 4.4: Estimação dos parâmetros do sistema de Lorenz baseada em sensibilidade de trajetória incluindo o acoplamento mestre-escravo e com ruído nas medidas.

\begin{tabular}{|c|c|c|c|c|c|}
\hline Parâmetro & $\begin{array}{c}\text { Valor } \\
\text { Inicial }\end{array}$ & $\begin{array}{c}\text { Desvio } \\
(\%)\end{array}$ & $\begin{array}{c}\text { Valor } \\
\text { Estimado }\end{array}$ & $\begin{array}{c}\text { Valor } \\
\text { Verdadeiro }\end{array}$ & $\begin{array}{c}\text { Erro } \\
(\%)\end{array}$ \\
\hline$\sigma$ & 4,60 & $-54 \%$ & 10,03 & 10,0 & 0,34 \\
\hline$r$ & 9,20 & $-54 \%$ & 19,96 & 20,0 & $-0,20$ \\
\hline$b$ & 4,10 & $+54 \%$ & 2,66 & 2,66 & 0,00 \\
\hline$z_{1 o}$ & 1,90 & $-5 \%$ & 2,07 & 2,00 & 3,50 \\
\hline$z_{2 o}$ & 2,85 & $-5 \%$ & 2,94 & 3,00 & $-2,00$ \\
\hline$z_{3 o}$ & 3,22 & $-54 \%$ & 6,96 & 7,00 & $-0,57$ \\
\hline
\end{tabular}
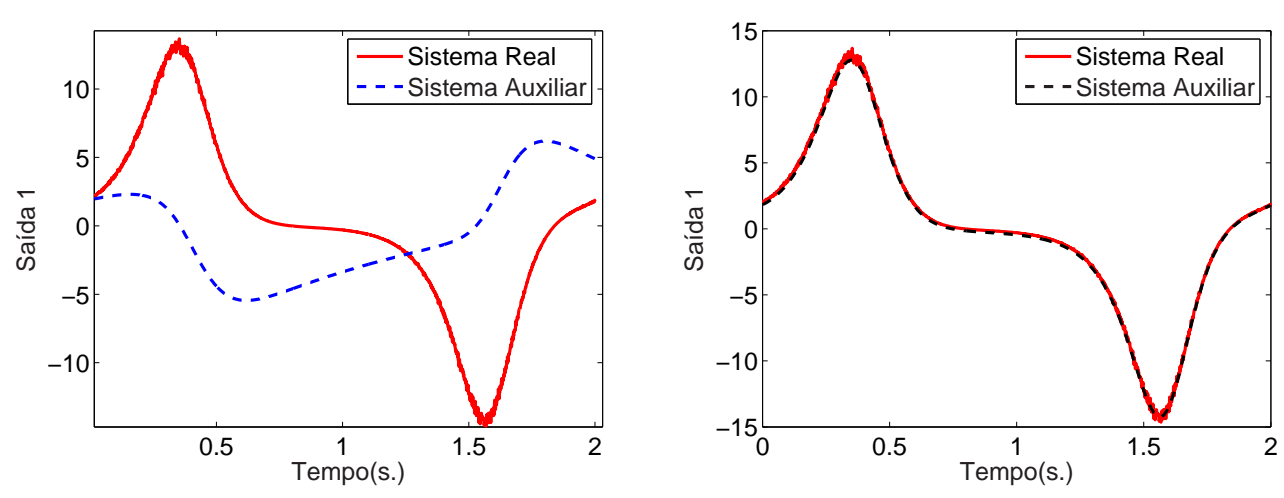

Figura 4.7: Saída \#1 antes e após a convergência dos parâmetros. Todas as medidas possuem ruído gaussiano de média zero e desvio padrão $5 \%$.

A convergência dos parâmetros ao longo das iterações pode ser visualizada na Figura 4.8. Foram necessárias 11 iterações até a convergência dos parâmetros e o tempo de cálculo foi $7 \mathrm{~s}$. para um computador Pentium IV.

\subsubsection{Mudando as entradas e as saídas}

Esta alternativa utiliza a metodologia de sensibilidade de trajetória na formulação tradicional, porém com uma configuração diferente das entradas e saídas. Neste caso supõe-se que $\left(x_{1}, x_{2}, x_{3}\right)$ são medidas. Dependendo da escolha da entrada, alguns parâmetros podem ser estimados. Por exemplo, utilizando $x_{2}$ como entrada e $x_{1}$ e $x_{3}$ como saídas, os parâmetros $\sigma$ e $b$ podem ser estimados. 

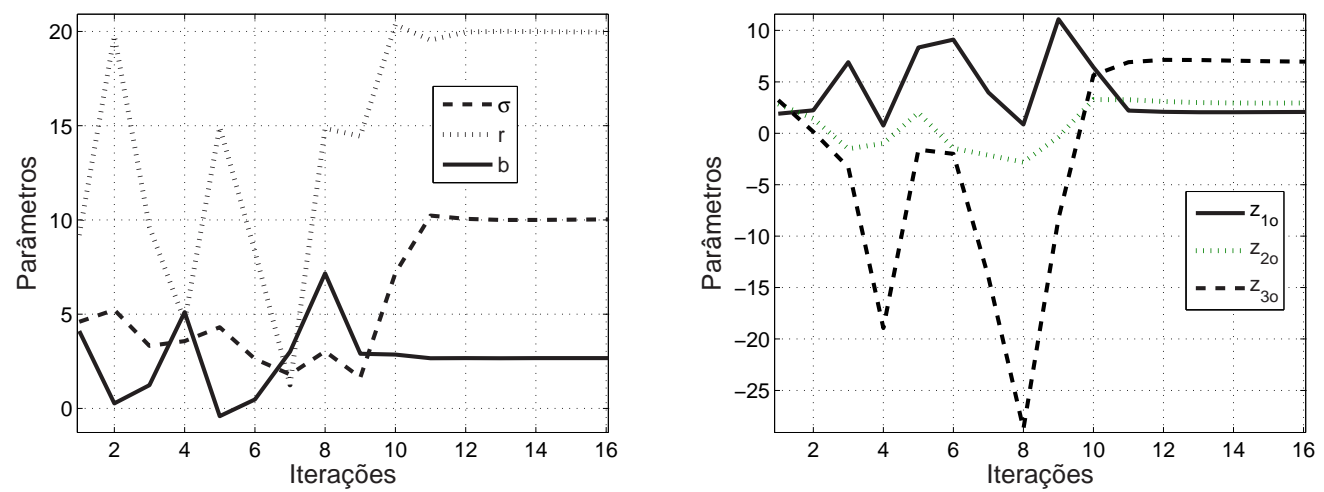

Figura 4.8: Convergência dos parâmetros ao longo das iterações.

O sistema auxiliar com esta configuração é:

$$
\begin{aligned}
& \dot{z}_{1}=\sigma x_{2}-\sigma z_{1} \\
& \dot{z}_{3}=z_{1} x_{2}-b z_{3} .
\end{aligned}
$$

As equações de sensibilidade são obtidas derivando-se (4.14) em relação aos parâmetros $\sigma$ e $b$.

Em relação a $\sigma$ :

$$
\begin{aligned}
& \dot{\lambda}_{z_{1}}^{\sigma}=x_{2}-z_{1}-\sigma \lambda_{z_{1}}^{\sigma}, \\
& \dot{\lambda}_{z_{3}}^{\sigma}=x_{2} \lambda_{z_{1}}^{\sigma}-b \lambda_{z_{3}}^{\sigma} .
\end{aligned}
$$

Em relação a $b$ :

$$
\begin{aligned}
& \dot{\lambda}_{z_{1}}^{b}=-\sigma \lambda_{z_{1}}^{b}, \\
& \dot{\lambda}_{z_{3}}^{b}=x_{2} \lambda_{z_{1}}^{b}-z_{3}-b \lambda_{z_{3}}^{b} .
\end{aligned}
$$

Na Figura 4.9 é apresentado o esquema de aplicação com esta abordagem.

O resumo dos resultados é mostrado na Tabela 4.5. A metodologia conseguiu estimar os parâmetros mesmo com alterações entre $-70 \%$ e $+70 \%$ em relação aos valores verdadeiros.

Os outros parâmetros $r$ e $z_{3 o}$ podem ser determinados considerando como entrada $x_{1}$ e como saídas $x_{2}$ e $x_{3}$ seguindo o mesmo procedimento.

O sucesso desta alternativa depende da possibilidade de escolher as entradas e saídas a fim de desacoplar os parâmetros estimados. Como foi nesta alternativa, 


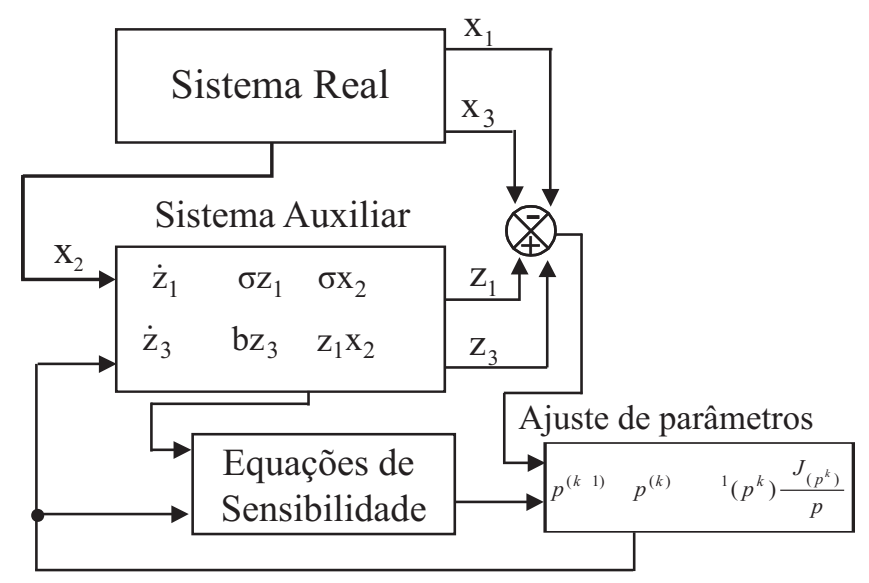

Figura 4.9: Estimação de parâmetros do sistema de Lorenz com a metodologia de sensibilidade de trajetória mudando as entradas e saídas.

Tabela 4.5: Estimação dos parâmetros do sistema de Lorenz com uma disposição diferente das entradas e saídas.

\begin{tabular}{|c|c|c|c|c|c|}
\hline Parâmetro & $\begin{array}{c}\text { Estimativa } \\
\text { inicial }\end{array}$ & $\begin{array}{c}\text { Desvio } \\
(\%)\end{array}$ & $\begin{array}{c}\text { Valor } \\
\text { final }\end{array}$ & $\begin{array}{c}\text { Valor } \\
\text { real }\end{array}$ & $\begin{array}{c}\text { Erro } \\
(\%)\end{array}$ \\
\hline$\sigma$ & 16,7 & $+67 \%$ & 10 & 10 & 0,0 \\
\hline $\mathrm{b}$ & 0,88 & $-67 \%$ & 2,66 & 2,66 & 0,0 \\
\hline
\end{tabular}

os parâmetros $\sigma$ e $b$ foram estimados em forma independente dos parâmetros $r$ e $z_{3 o}$.

\subsection{Estimação de Parâmetros do Sistema de Chua}

Seja o sistema de Chua (Chua et al., 1993a) modelado por :

$$
\begin{aligned}
\dot{x_{1}} & =\alpha\left(x_{2}-x_{1}-f\left(x_{1}\right)\right) \\
\dot{x_{2}} & =x_{1}-x_{2}+x_{3} \\
\dot{x_{3}} & =-\beta x_{2}-\gamma x_{3} \\
f\left(x_{1}\right) & =b x_{1}+\frac{1}{2}(a-b)\left\{\left|x_{1}+1\right|-\left|x_{1}-1\right|\right\}
\end{aligned}
$$

em que $\alpha, \beta$ e $\gamma, a$ e $b$ são os parâmetros a serem estimados. Neste caso, admite-se que todos os estados são observáveis, isto é $y=\left(x_{1}, x_{2}, x_{3}\right)^{T}$ é o vetor de saída do sistema real. Além disso, considera-se que as condições iniciais das equações 
diferenciais do modelo são desconhecidas e precisam ser estimadas. Assim, o vetor de parâmetros é $p=\left(\alpha, \beta, \gamma, a, b, x_{1 o}, x 2 o, x 3 o\right)$, cujos valores são $\alpha_{r}=6,5729$, $\beta_{r}=10,9024, \gamma_{r}=-0,0445, a=-1,1829, b=-0,6524, x_{1 o}=0,15, x_{2 o}=0,90$, $x_{3 o}=0,80$. As medidas do sistema real foram obtidas via integração numérica de (4.17) com seus parâmetros e condições iniciais verdadeiros. Além disso, todas as medidas estão sujeitas a ruído gaussiano de média zero e desvio padrão $5 \%$.

Da mesma forma que o sistema de Lorenz, os parâmetros do sistema de Chua não podem ser estimados simultaneamente usando o metodologia de sensibilidade de trajetória. Testes computacionais mostram que os parâmetros não convergem para erros nos valores iniciais dos parâmetros de até 1\%. A fim de contornar esta dificuldade, o seguinte sistema auxiliar foi escolhido.

$$
\begin{aligned}
\dot{z_{1}} & =\alpha\left(z_{2}-x_{1}-f\left(x_{1}\right)\right)-k\left(z_{1}-x_{1}\right) \\
\dot{z_{2}} & =x_{1}-z_{2}+z_{3} \\
\dot{z_{3}} & =-\beta z_{2}-\gamma z_{3} \\
f\left(z_{1}\right) & =b x_{1}+\frac{1}{2}(a-b)\left\{\left|z_{1}+1\right|-\left|z_{1}-1\right|\right\}
\end{aligned}
$$

O sistema auxiliar é similar ao modelo do sistema de Chua, a diferença é que foi usado o acoplamento mestre-escravo, isto é, a variável $z_{1}$ foi substituída pela medida $x_{1}$. Além disso, para aumentar a robustez da sincronização das saídas, um termo adicional foi incluído à primeira equação de (4.18), $g\left(z_{1}, x_{1}\right)=$ $k\left(z_{1}-x_{1}\right)$, com $\mathrm{k}=10$. Testes computacionais mostraram que este termo melhora a estabilidade do sistema, contribuindo na sincronização das saídas.

A Figura 4.10 mostra o diagrama esquemático da aplicação da metodologia. O bloco "Equações de Sensibilidade" está formado pelas equações obtidas pelas derivadas de (4.18) em relação a cada parâmetro. O bloco "Ajuste de Parâmetros" atualiza os parâmetros em cada iteração. A matriz $\Gamma$ e o vetor $\frac{\partial J(p)}{\partial p}$ podem ser determinados a partir da solução do sistema auxiliar e das equações de sensibilidade usando (2.25) e (2.27), respectivamente, segundo procedimento da Seção 2.6 .

Os resultados da estimação estão mostrados na Tabela (4.6). A metodologia conseguiu estimar os parâmetros mesmo com alterações entre $-65 \%$ e $+65 \%$ em 


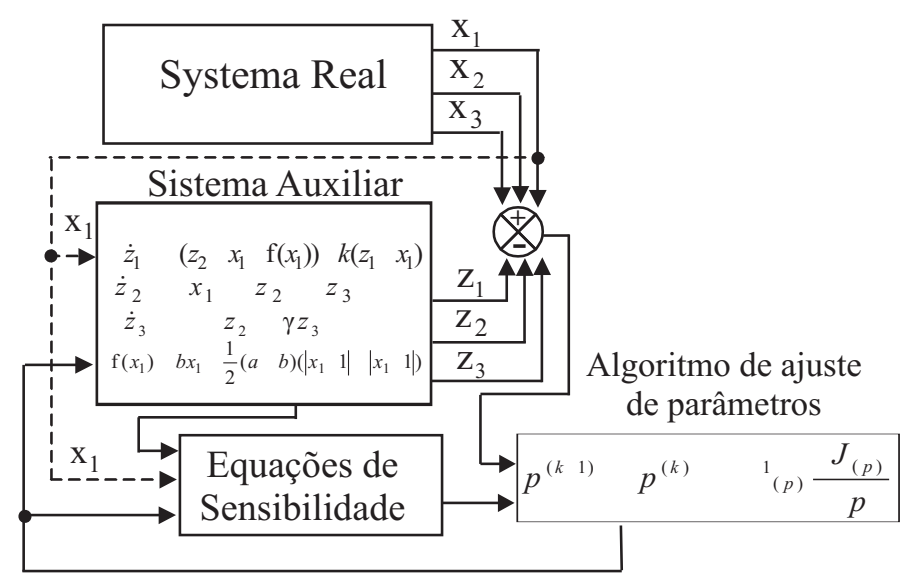

Figura 4.10: Estimação de parâmetros do sistema de Chua baseada em sensibilidade de trajetória incluindo o acoplamento mestre-escravo.

relação aos valores verdadeiros.

Tabela 4.6: Estimação dos parâmetros do sistema de Chua utilizando sensibilidade de trajetória incluindo o acoplamento mestre-escravo.

\begin{tabular}{|c|c|c|c|c|c|}
\hline Parâmetro & $\begin{array}{c}\text { Valor } \\
\text { inicial }\end{array}$ & Desvio & $\begin{array}{c}\text { Valor } \\
\text { final }\end{array}$ & $\begin{array}{c}\text { Valor } \\
\text { real }\end{array}$ & $\begin{array}{c}\text { Erro } \\
(\%)\end{array}$ \\
\hline$\alpha$ & 2,3027 & $-65 \%$ & 6,5435 & 6,5792 & 0,54 \\
\hline$\beta$ & 3,8158 & $-65 \%$ & 10,8906 & 10,9024 & 0,11 \\
\hline$\gamma$ & $-0,0156$ & $-65 \%$ & $-0,0444$ & $-0,0445$ & 0,27 \\
\hline$a$ & $-0,4137$ & $-65 \%$ & $-1,1807$ & $-1,1820$ & 0,12 \\
\hline$b$ & $-0,2283$ & $-65 \%$ & $-0,6539$ & $-0,6524$ & 0,24 \\
\hline$x_{0}$ & 0,0525 & $-65 \%$ & 0,1542 & 0,1500 & 2,83 \\
\hline$y_{0}$ & 0,3150 & $-65 \%$ & 0,9009 & 0,9000 & 0,10 \\
\hline$z_{0}$ & 0,2800 & $-65 \%$ & 0,8185 & 0,8000 & 2,31 \\
\hline
\end{tabular}




\section{Capítulo 5}

\section{Estimação de Parâmetros do Gerador Síncrono}

\subsection{Introdução}

Este capítulo apresenta a aplicação da metodologia de estimação de parâmetros em geradores síncronos visando a implementação em sistemas reais. Para isto, é necessário contornar várias dificuldades práticas. Estas dificuldades surgem porque a estimação dos parâmetros da máquina síncrona deve satisfazer certos requisitos práticos, tais como a obtenção dos parâmetros com a máquina ligada ao sistema e a utilização apenas de medidas locais e de fácil acesso.

Os modelos da máquina síncrona são equações diferenciais não-lineares que possuem grandezas que não podem ser medidas diretamente, tais como as tensões transitórias de eixo direto e em quadratura $\left(E_{d}^{\prime}(t)\right.$ e $\left.E_{q}^{\prime}(t)\right)$, ou variáveis de difícil obtenção tal como o ângulo do rotor $(\delta(t))$. Além disso, as não-linearidades das equações do gerador fazem com que o sucesso da estimação dependa fortemente dos valores iniciais dos parâmetros. Se estes não estiverem suficientemente próximos, os parâmetros não podem ser estimados. Na próxima seção detalhamse estas dificuldades e são propostas algumas alternativas de solução. 


\subsection{Dificuldades e alternativas de solução}

Esta seção apresenta as diferentes dificuldades no momento de estimar os parâmetros da máquina síncrona a fim de propor uma metodologia que evite tais dificuldades.

1. Obtenção das medidas com o gerador em operação: As medidas necessárias para a estimação dos parâmetros devem ser obtidas com o gerador síncrono ligado ao sistema, pois, desligar o gerador do SEP resulta em grande prejuízo à empresa de geração.

Os testes tradicionais, usualmente realizados para obtenção das medidas, são testes com a máquina desconectada do SEP (testes off-line), como por exemplo o teste no domínio da freqüência com rotor parado (stand still frequency response) (IEEE, 1995), que apenas podem ser realizados durante a manutenção programada do gerador. Isto limita a aplicação da metodologia em sistemas reais. Além disso, os parâmetros da máquina síncrona obtidos de testes off-line podem ser diferentes daqueles quando a máquina é colocada em operação.

Na literatura são relatados também testes que são realizados com a máquina conectado ao SEP (testes on-line). Alguns destes testes estão baseados na injeção de uma seqüência de sinais (seqüência binária pseudoaleatória, PRBS) (Karrari e Malik, 2004; Vermeulen et al., 2002) ou apenas de um pequeno sinal no sistema de excitação, a fim de excitar a resposta dinâmica da máquina síncrona (Karaagac e Tor, 2006), (Le e Wilson, 1988), (Velez e Lida, 2001), (Burth et al., 1999) e (Zhengming et al., 1995). As metodologias baseadas nesses tipos de testes geralmente utilizam o modelo linearizado da máquina síncrona. O problema desta abordagem é que o sinal injetado pode não ter informação suficiente para obter a resposta dinâmica da máquina síncrona e, o mais grave ainda, pode causar instabilidade no sistema. Além disso, a realização desse teste necessita da aprovação por parte do centro de operação do SEP. 
Para contornar este problema propõe-se:

(a) Utilizar medidas obtidas a partir de oscilografias de perturbações: A proposta é utilizar as oscilografias de relés digitais. Assim, a máquina não precisa ser desligada do sistema, sendo apenas necessário esperar um evento do SEP (falta em uma linha de transmissão) para que os relés registrem a informação.

(b) Utilizar o modelo não-linear da máquina síncrona: Mais que uma alternativa, isto é uma necessidade da proposta anterior. Para poder utilizar dados de oscilografias de grandes perturbações, a máquina síncrona deve ser modelada na sua forma não-linear.

\section{Os modelos dependem dos parâmetros da rede e possuem variáveis}

não-mensuráveis: Os modelos da máquina síncrona, amplamente utilizados para estimação de parâmetros, são aqueles usados em estudos de estabilidade do SEP (IEEE, 2002). Estes modelos possuem parâmetros da rede e variáveis que não são mensuráveis.

Os parâmetros da rede presentes nas equações, são os parâmetros das linhas de transmissão (resistências e reatâncias) e a tensão na barra de referência (barramento infinito). Algumas pesquisas (Burth et al., 1999; Zhengming et al., 1995) aplicam algumas técnicas de regressão linear para estimar estes parâmetros. O problema de utilizar modelos que dependem dos parâmetros da rede é que os erros destes podem espalhar-se na precisão dos parâmetros estimados do gerador.

Os modelos também possuem variáveis que não podem ser medidas diretamente, como, por exemplo, as tensões transitórias de eixo direto e em quadratura $\left(E_{d}^{\prime}(t)\right.$ e $E_{q}^{\prime}(t)$, respectivamente). Para contornar este problema as seguintes estratégias são adotadas:

(a) Utilizar a tensão terminal como referência: A máquina síncrona será modelada usando como referência a barra terminal, com isso os parâmetros 
do gerador podem ser estimados de forma independente dos parâmetros da rede.

(b) Estimar as condições iniciais $E_{d o}^{\prime}$ e $E_{q o}^{\prime}$ pela metodologia de estimação.

3. Os modelos dependem do ângulo de rotor $(\delta(t))$ : Os modelos do gerador síncrono dependem do ângulo de rotor, que é uma variável difícil de ser medida em sistemas reais. Usualmente, esta variável é estimada integrando a velocidade angular $\omega(t)$, porém, é necessário conhecer o valor exato do ângulo de rotor em estado estacionário $\delta_{o}$, o qual não é uma tarefa trivial e, além disso, o sucesso da estimação dos parâmetros fica muito dependente desse valor.

Em (de Mello, 1994), o ângulo de rotor é medido utilizando as componentes simétricas das tensões e correntes da máquina síncrona e em (Chen et al., 2000) utilizando um sensor posicionado no eixo do rotor e um GPS (global position system) para sincronizar as medidas. O PMU (phasor measurement unit) pode ser usado para medir o ângulo da tensão terminal em relação à referência síncrona, porém é insuficiente para medir ângulo de rotor. um tipo especial de PMU denominado APMU (angle phasor measuremt unit), proposto em (Jin et al., 2007), pode ser usado para medir $\delta(t)$ diretamente a partir da velocidade angular. Entretanto, o uso deste dispositivo em SEP ainda não é muito comum, além de ser caro.

A fim de contornar esta dificuldade serão testadas as seguintes alternativas.

(a) Estimar o ângulo do rotor $(\delta(t))$ a partir da velocidade ângular $\omega(t)$ e considerar a condição inicial $\delta_{o}$ como parâmetro a ser estimado pela metodologia. Neste caso utilizar-se-á o acoplamento mestre-escravo para aumentar a região da convergência.

(b) Transformar as equações originais em equações equivalentes com variáveis que possam ser medidas com maior facilidade.

(c) Adicionar uma equação algébrica no modelo do gerador que relacione o ângulo de rotor com as outras variáveis do modelo. Esta equação 
algébrica relaciona o ângulo de rotor com relação a tensão terminal (ângulo de potência). Este ângulo pode ser calculado numericamente por intermédio desta equação.

\section{A metodologia de estimação possui uma região de convergência} pequena: O sucesso da estimação dos parâmetros depende em grande parte dos valores iniciais assumidos pelos parâmetros, isto é, se estes estiverem próximos dos valores verdadeiros, há uma probabilidade maior do processo de estimação ser completado com sucesso. A fim de garantir o sucesso da estimação, os valores iniciais devem estar contidos dentro da região de convergência da metodologia. Os valores iniciais dos parâmetros podem ser obtidos a partir dos dados do fabricante ou valores padronizados de unidades similares. Segundo a experiência na estimação de parâmetros do gerador, adquirida neste trabalho, se quisermos estimar todos os parâmetros do gerador simultaneamente, a região de convergência pode ser tão pequena que não serviria em aplicações práticas. Para contornar esta dificuldade, inicialmente foram estimados os parâmetros de modelos simplificados, e pouco a pouco foi aumentado o grau de detalhamento da máquina. Como referência, vide (Cari, 2005), onde foram estimados os parâmetros dos modelos simplificados.

As seguintes alternativas são propostas neste trabalho:

(a) Estimar os parâmetros mecânicos e elétricos em forma desacoplada. Isto é conseguido escolhendo convenientemente as entradas e saídas do modelo.

(b) Incluir o acoplamento mestre-escravo. Como foi demonstrado na seção 3.2, o acoplamento é escolhido de modo a diminuir termos não-lineares e ajudar na sincronização das saídas.

(c) Utilizar a abordagem de minimização para eliminar problemas de singularidade das EADs e permitir a estimação mesmo quando os parâmetros estiverem distantes dos valores verdadeiros. 
(d) Estimar os parâmetros em duas fases. Para isto, realiza-se uma análise de sensibilidade a fim de determinar a influência dos parâmetros na saída.

5. Problemas de mal-condicionamento: Os problemas de mal-condicionamento do processo de estimação possuem basicamente duas origens: (i) as medidas obtidas do sistema real não contêm informações suficientes para a estimação dos parâmetros, (ii) as saídas são muito sensíveis a alguns parâmetros e pouco sensíveis a outros.

No primeiro caso, se as medidas não tiverem informação suficiente, é necessário repetir o ensaio para obter medidas com maior informação da resposta dinâmica. No segundo caso, o acoplamento mestre-escravo e o algoritmo de estimação em duas fases minimiza o efeito deste problema.

O resumo das dificuldades encontradas para estimar os parâmetros de geradores síncronos utilizando a técnica de sensibilidade de trajetória e as propostas para superar estas dificuldades são apresentadas na Figura 5.1.

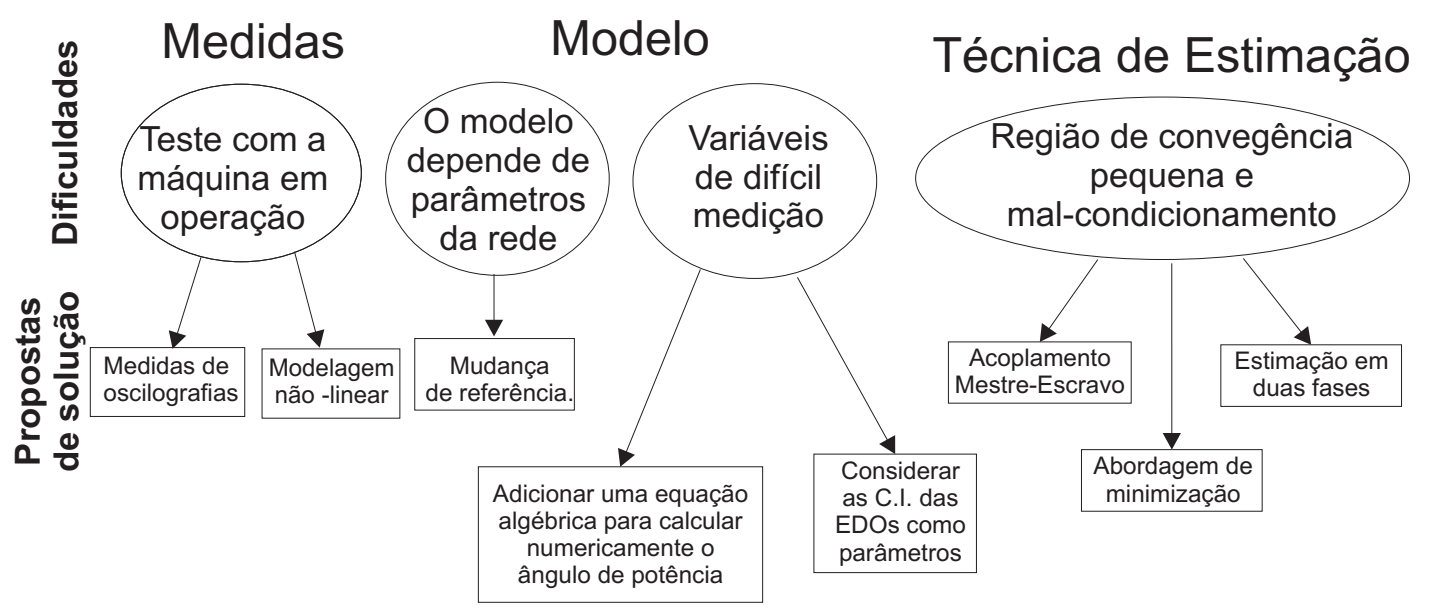

Figura 5.1: Dificuldades e propostas de solução para a estimação dos parâmetros da máquina síncrona 


\subsection{Esquema Para Estimar os Parâmetros com o Gerador em Operação}

O esquema proposto para estimar os parâmetros a partir de medidas de perturbações obtidas com o gerador em operação (on-line ${ }^{1}$ ) é mostrado na Figura 5.2 . Inicialmente, registram-se as medidas disponíveis do sistema real. Estas medidas são obtidas das oscilografias de relés ou diretamente de medidores instalados em lugares estratégicos durante uma perturbação no SEP, caso ocorra. No caso da máquina síncrona, estas medidas são as tensões e correntes trifásicas, a saída do sistema de excitação e a velocidade mecânica do rotor (caso queira-se estimar os parâmetros mecânicos). Estas medidas passam por uma etapa de filtragem de ruído e formarão parte do bloco "Sistema Real". Algumas das medidas do sistema real são selecionadas como entrada e outras como saída deste. Quanto maior for a quantidade de medidas do banco de dados, maior será a possibilidade de realizar a validação dos parâmetros estimados.

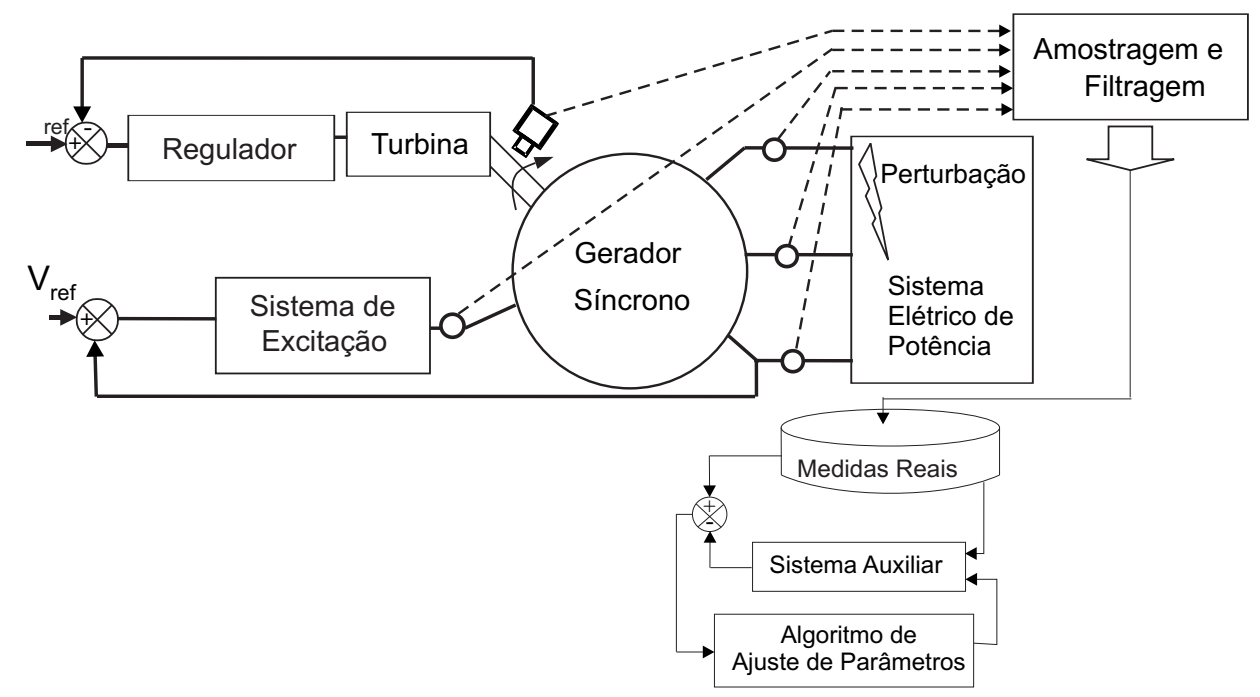

Figura 5.2: Estratégia para estimação de parâmetros. As medidas são obtidas durante uma perturbação no SEP e utilizadas pela metodologia de estimação de parâmetros.

As equações do gerador síncrono compõem o bloco "Sistema Auxiliar". A

\footnotetext{
${ }^{1} \mathrm{O}$ termo on-line não implica que a aplicação é realizada em tempo real (real-time), porém que é realizada com a máquina síncrona conectada ao SEP ou em operação.
} 
diferença das saídas dos dois sistemas é minimizada utilizando o algoritmo iterativo de ajuste de parâmetros apresentado na Seção 2.6. Esta estratégia satisfaz os requisitos práticos para a aplicação da metodologia em um sistema real.

\subsubsection{Considerações práticas para a realização dos testes}

Os testes aqui neste trabalho são obtidos através de simulações, porém a experiência adquirida com estes testes pode ser utilizada em futuros testes em sistemas reais como mostra a figura 5.2.

Outra consideração é que na presença de uma perturbação no sistema existem três períodos bem definidos: período pré-falta (ou condições de operação normal), período em falta (na presença da perturbação) e período pós-falta (depois de eliminada a perturbação). O processo de estimação é realizado alguns ciclos após o começo do período pós-falta. Isto pode ser visualizado na Figura (5.3). Iniciar a análise alguns ciclos após à eliminação da falta não é extremamente necessário mas acreditamos que problemas de ruído e chaveamentos são minimizados.

Algo importante que pode observar-se na Figura (5.3) é que os valores inicias das variáveis de estado no começo do processo de estimação pode ser totalmente diferente aos seus valores em regime permanente. Isto dificulta a obtenção de $E_{q o}^{\prime}$ e $E_{d o}^{\prime}$ no começo do processo de estimação a partir de estimativas de regime permanente.

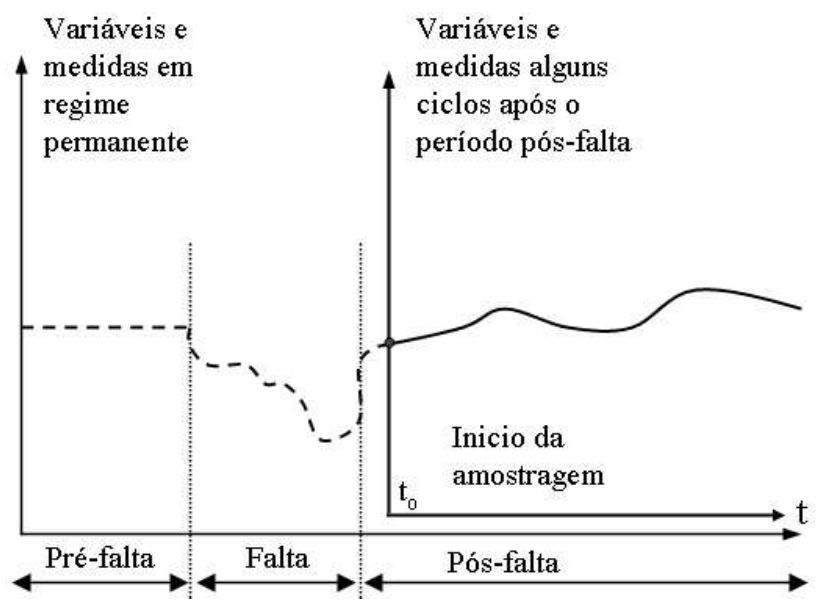

Figura 5.3: Condições de Operação no processo de estimação. 


\subsubsection{Sistema de potência}

O sistema de potência para o estudo é constituído por um gerador equivalente de uma planta (4x555 MVA), $24 \mathrm{kV}$ e $60 \mathrm{~Hz}$ que fornece potência a um barramento infinito através de duas linhas de transmissão como mostra a Figura $\underline{5.4}$ (Kundur, 1994). Os valores das impedâncias de linha, do transformador, das potência e tensões estão dados em valores por unidade (pu). A potência base é 2200 MVA, as tensões base no lado de baixa e alta tensão são $24 \mathrm{kV}$ e $220 \mathrm{kV}$ respectivamente. O gerador síncrono tem um sistema de excitação tipo ST1A (IEEE, 1992) com os parâmetros dados em pu.: $K_{A}=200, T_{A}=0.025, E f d_{\min }=-6.4$ e $E f d_{\max }=7$.

O evento no SEP, para obtenção das medidas, consiste em um curto-circuito trifásico em um dos terminais da linha de transmissão $L_{2}$. A falta é eliminada pela atuação dos dispositivos de proteção que isola a linha em falta em $t=0,07 \mathrm{~s}$. Os dados foram gerados a partir de simulações resolvendo as equações do sistema auxiliar com os parâmetros verdadeiros e usando o programa Matlab 6.0.

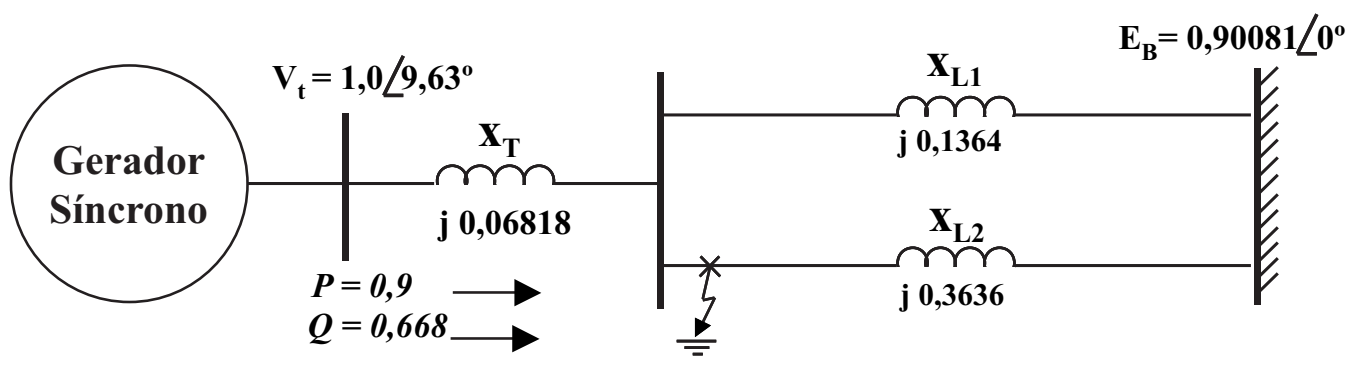

Figura 5.4: Sistema de potência usado para os testes. As medidas são coletadas durante uma perturbação no SEP.

\subsubsection{Sistema de referência}

Dois sistemas de referências são utilizados para modelar o SEP. A máquina síncrona é modelada na referência $d-q$ que fica posicionada no rotor e gira a velocidade do rotor. Um dos eixos fica alinhado com o eixo magnético do enrolamento de campo, conhecido como eixo direto, e o outro eixo fica atrasado $90^{\circ}$ em relação ao enrolamento de campo e é conhecido como eixo em quadratura. As grandezas 
do estator são projetadas na referência $d-q$ por intermédio da transformação de Park (Padiyar, 1996). A rede externa é modelada na referência $I_{m}-R_{e}$ (referência síncrona), com o eixo $R_{e}$ atrasado $90^{\circ}$ do eixo $I_{m}$. Para simplificar a análise, a tensão de referência $E_{B}$ coincide com o eixo $R_{e}$.

Na Figura 5.5, mostram-se os dois sistemas de referências. $\delta$ e $\theta$ são os ângulos do eixo em quadratura e da tensão terminal, respectivamente, em relação à referência síncrona. Além disso, $\phi$ é o ângulo de fator de potência.

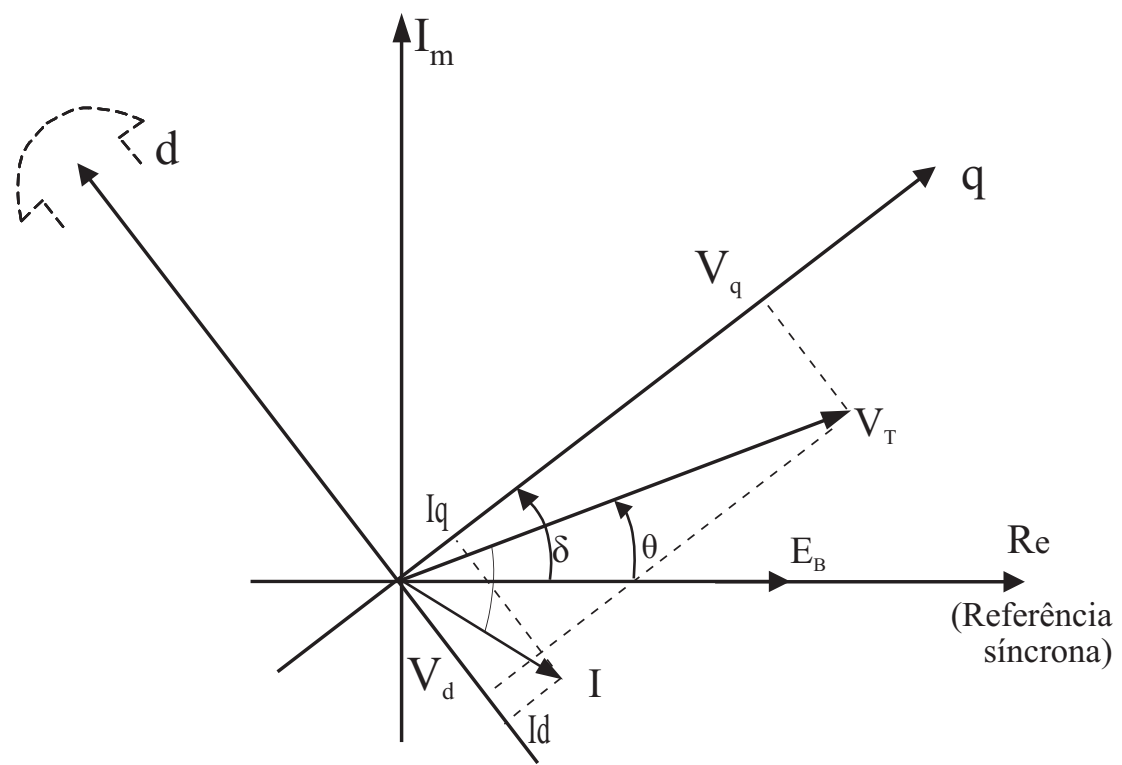

Figura 5.5: Sistemas de referência, da máquina síncrona $(d-q)$ e da rede externa $\left(I_{m}-R_{e}\right)$

\subsubsection{Modelo Original do Gerador Síncrono}

Considere as equações diferenciais do modelo de dois-eixos da máquina síncrona (Padiyar, 1996):

$$
\begin{aligned}
\dot{\delta} & =\omega \\
\dot{\omega} & =\frac{\omega_{o}}{2 H}\left[P_{m}-E_{q}^{\prime} I_{q}-E_{d}^{\prime} I_{d}-\left(x_{d}^{\prime}-x_{q}^{\prime}\right) I_{d} I_{q}-\frac{D}{\omega_{o}} \omega\right], \\
\dot{E}_{q}^{\prime} & =\frac{1}{T_{d o}^{\prime}}\left[E_{f d}-E_{q}^{\prime}+\left(x_{d}-x_{d}^{\prime}\right) I_{d}\right], \\
\dot{E}_{d}^{\prime} & =\frac{-1}{T_{q o}^{\prime}}\left[E_{d}^{\prime}+\left(x_{q}-x_{q}^{\prime}\right) I_{q}\right],
\end{aligned}
$$




$$
\begin{aligned}
I_{d} & =\frac{E_{B} \cos (\delta)-E_{q}^{\prime}}{x_{d}^{\prime}+Z_{I}} \\
I_{q} & =\frac{E_{B} \operatorname{sen}(\delta)+E_{d}^{\prime}}{x_{q}^{\prime}+Z_{I}} .
\end{aligned}
$$

onde $I_{d}$ (corrente em eixo direto) e $I_{q}$ (corrente em eixo em quadratura) são variáveis intermediárias, $E_{B}$ é a magnitude de tensão na barra de referência e $Z_{I}=x_{T}+x_{L 1}$ é a impedância série entre a tensão de referência $\left(E_{B}\right)$ e a tensão terminal $\left(V_{T}\right)$ em pós-falta (Vide Figura 5.4).

Os parâmetros elétricos do gerador síncrono são:

$x_{d}$ : Reatância de eixo direto.

$x_{q}$ : Reatância de eixo em quadratura.

$x_{d}^{\prime}$ : Reatância transitória de eixo direto.

$x_{q}^{\prime}$ : Reatância transitória de eixo em quadratura.

$T_{d o}^{\prime}:$ Constante de tempo de eixo direto em circuito aberto.

$T_{q o}^{\prime}$ : Constante de tempo de eixo em quadratura em circuito aberto.

Além destes parâmetros, o gerador síncrono possui dois parâmetros mecânicos: a constante de inércia $H$ e a constante de amortecimento $D$.

Este modelo, usado amplamente em estudos de estabilidade transitória em caso de máquinas de rotor de pólos lisos, possui 4 variáveis de estado $\left(\delta, \omega, E_{q}^{\prime}, E_{d}^{\prime}\right)$ e 8 parâmetros $\left(x_{d}, x_{d}^{\prime}, T_{d o}^{\prime}, x_{q}, x_{q}^{\prime}, T_{q o}^{\prime}, H, D\right)$. O inconveniente deste modelo é que, além de depender dos parâmetros da rede $\left(E_{B}, Z_{I}\right)$, ele requer o conhecimento dos estados $E_{q}^{\prime}(t)$ e $E_{d}^{\prime}(t)$ que não podem ser medidos e de $\delta(t)$ que é uma medida difícil de ser obtida na prática. Uma alternativa para contornar este problema seria estimar com a metodologia as condições iniciais destas variáveis $\left(E_{q o}^{\prime}, E_{d o}^{\prime}, \delta_{o}\right)$ como parâmetros. Assim, o número de parâmetros a serem estimados aumenta para 11. Embora este modelo não permita o cálculo dos parâmetros sub-transitórios, tais como $x_{d}^{\prime \prime}, T_{d o}^{\prime \prime}, x_{q}^{\prime \prime}, T_{q o}^{\prime \prime}$, ele será utilizado por simplicidade.

É necessário um estudo adicional para aplicar a metodologia proposta para modelos sub-transitórios. Em geral, quanto maior é o número de parâmetros 
a serem estimados simultaneamente, pior fica o condicionamento numérico da metodologia. Além disso, está mais suscetível à existência de múltiplas soluções devido a natureza não-convexa do problema.

Na tentativa de estimar os 11 parâmetros simultaneamente usando este modelo, observou-se que a convergência só ocorre quando o vetor de parâmetros inicial estiver muito perto do valor verdadeiro. Com o objetivo de contornar este problema intrínseco do modelo e diminuir o número de parâmetros que são simultaneamente estimados, propõe-se desacoplar o modelo para realizar a estimação dos parâmetros mecânicos e elétricos de forma independente. Este desacoplamento realiza-se escolhendo as entradas e saídas em forma apropriada para a estimação dos parâmetros elétricos e mecânicos, apresentado nas seções subsequentes.

\subsection{Estimação dos Parâmetros Mecânicos do Ge- rador}

Considere a equação (5.2)

$$
\dot{\omega}=\frac{\omega_{o}}{2 H}\left[P_{m}-E_{q}^{\prime} I_{q}-E_{d}^{\prime} I_{d}-\left(x_{d}^{\prime}-x_{q}^{\prime}\right) I_{d} I_{q}-\frac{D}{\omega_{o}} \omega\right] .
$$

Substituindo $P_{e}=E_{q}^{\prime} I_{q}+E_{d}^{\prime} I_{d}+\left(x_{d}^{\prime}-x_{q}^{\prime}\right) I_{d} I_{q}$ na equação anterior, obtém-se

$$
\dot{\omega}=\frac{\omega_{o}}{2 H}\left[P_{m}-P_{e}-\frac{D}{\omega_{o}} \omega\right]
$$

A equação (5.7) é utilizada para estimar os parâmetros mecânicos do gerador. A potência ativa $P_{e}$, calculada a partir das tensões e correntes trifásicas, é considerada como entrada e $\omega$ como saída. Este modelo é completamente desacoplado das variáveis e parâmetros elétricos e contém uma variável de estado $\omega$ e dois parâmetros $p=(H, D)^{T}$.

O parâmetro " $D$ " pode depender das variações da velocidade em relação ao valor nominal. Entretanto, como a potência elétrica é uma medida de entrada, os efeitos de amortecimento devido ao enrolamento amortecedor são automati- 
camente considerados e não influenciam a estimação de " $D$ ". Neste caso, " $D$ " representa apenas os efeitos de amortecimento mecânico.

\subsubsection{Equações de sensibilidade}

As equações de sensibilidade são obtidas derivando-se (5.7) em relação aos parâmetros, isto é:

$$
\begin{aligned}
\dot{\lambda}_{\omega}^{H} & =\frac{-\omega_{o}}{2 H^{2}}\left(P_{m}-P_{e}-\frac{D}{\omega_{o}} \omega\right)+\frac{\omega_{o}}{2 H}\left(\frac{-D}{\omega_{o}} \lambda_{\omega}^{H}\right), \\
\dot{\lambda}_{\omega}^{D} & =\frac{\omega_{o}}{2 H}\left(-\frac{\omega}{\omega_{o}}-\frac{D}{\omega_{o}} \lambda_{\omega}^{D}\right),
\end{aligned}
$$

sendo $\lambda_{\omega}^{H}=\frac{\partial \omega}{\partial H}$ e $\lambda_{\omega}^{D}=\frac{\partial \omega}{\partial D}$.

As condições iniciais do sistema (5.7) são: $\lambda_{\omega}^{H}\left(t_{o}\right)=\lambda_{\omega}^{D}\left(t_{o}\right)=0$. A sensibilidade da saída pode ser obtida por substituição direta como: $\frac{\partial \omega}{\partial p}=\left(\lambda_{\omega}^{H}, \lambda_{\omega}^{D}\right)^{T}$.

Na Figura 5.6 mostra-se o diagrama de blocos para estimar os parâmetros mecânicos. O bloco sistema auxiliar contém as equações do modelo. O ajuste dos parâmetros é realizado calculando $\Gamma$ e $\frac{\partial J(p)}{\partial p}$ usando as equações $(2.25)$ e (2.27), respectivamente.

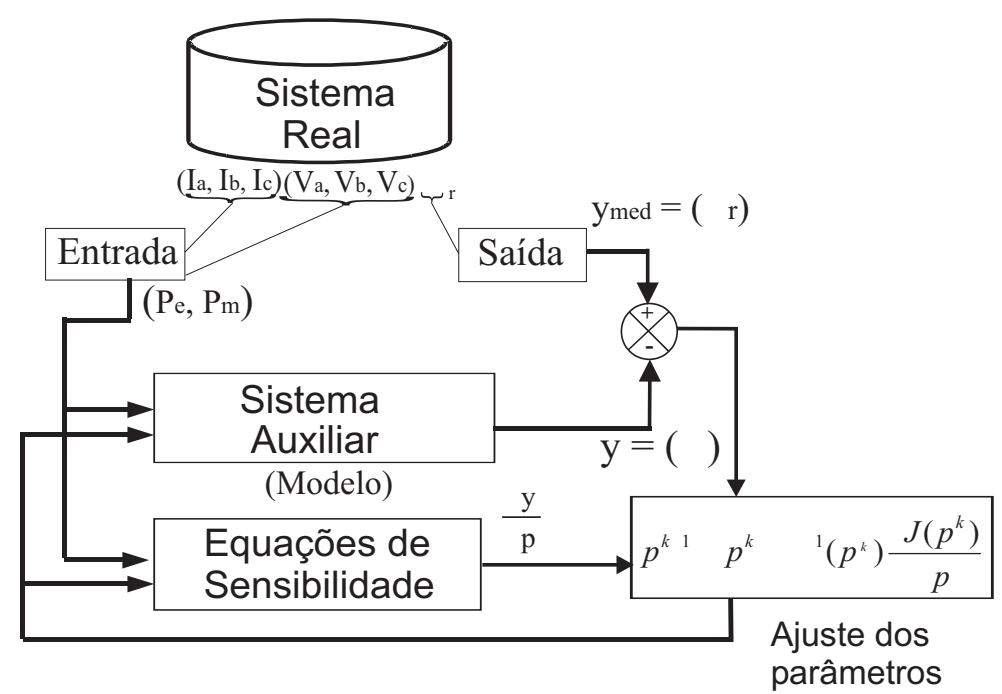

Figura 5.6: Diagrama de blocos para a estimação dos parâmetros mecânicos. 


\subsubsection{Resultados}

A metodologia conseguiu estimar os parâmetros mesmo com alterações entre $90 \%$ e $+90 \%$ em relação aos valores verdadeiros. Os parâmetros convergiram a seus valores verdadeiros após 7 iterações como é mostrado na Tabela 5.1 .

Tabela 5.1: Estimação dos parâmetros mecânicos.

\begin{tabular}{|c|c|c|c|c|c|}
\hline Parâmetro & $\begin{array}{c}\text { Valor } \\
\text { Inicial }\end{array}$ & Alteração & $\begin{array}{c}\text { Valor } \\
\text { Final }\end{array}$ & $\begin{array}{c}\text { Valor } \\
\text { Verdadeiro }\end{array}$ & Erro (\%) \\
\hline$H$ & 6,650 & $+90 \%$ & 3,500 & 3,500 & 0,000 \\
\hline$D$ & 0,090 & $-90 \%$ & 0,899 & 0,900 & $-0,111$ \\
\hline
\end{tabular}

\subsubsection{Influência do ruído nas medidas}

A metodologia de sensibilidade de trajetória foi aplicada para estimar os parâmetros mecânicos sob a influência de ruído. Para este propósito, ruído Gaussiano de média zero e desvio padrão $1 \%$ foi adicionado em todas as medidas.

Na Tabela 5.2, mostram-se os resultados dos parâmetros com a inclusão do ruído. Os resultados mostram que a região de convergência não é afetada pelo ruído nas medidas e a precisão dos parâmetros estimados foi levemente modificada.

Tabela 5.2: Estimação dos parâmetros mecânicos com ruído nas medidas.

\begin{tabular}{|c|c|c|c|c|c|}
\hline parâmetro & $\begin{array}{l}\text { Valor } \\
\text { Inicial }\end{array}$ & Alteração & $\begin{array}{l}\text { Valor } \\
\text { Final }\end{array}$ & $\begin{array}{c}\text { Valor } \\
\text { Verdadeiro }\end{array}$ & $\operatorname{Erro}(\%)$ \\
\hline$H$ & 6,650 & $+90 \%$ & 3,504 & 3,500 & 0,114 \\
\hline$D$ & 0,090 & $-90 \%$ & 0,860 & 0,900 & $-4,444$ \\
\hline
\end{tabular}

A Figura 5.7 mostra a convergência dos parâmetros ao longo das iterações. Foram necessárias 8 iterações para atingir convergência aos valores corretos. $\mathrm{Na}$ Figura 5.8 mostra-se a diferença entre as saídas do sistema real e auxiliar.

Na seqüência, detalha-se a aplicação da metodologia de estimação para estimar os parâmetros elétricos do gerador. 


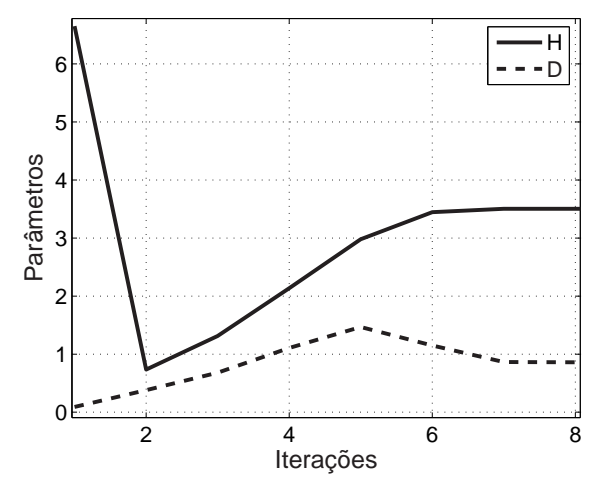

Figura 5.7: Convergência dos parâmetros mecânicos ao longo das iterações.

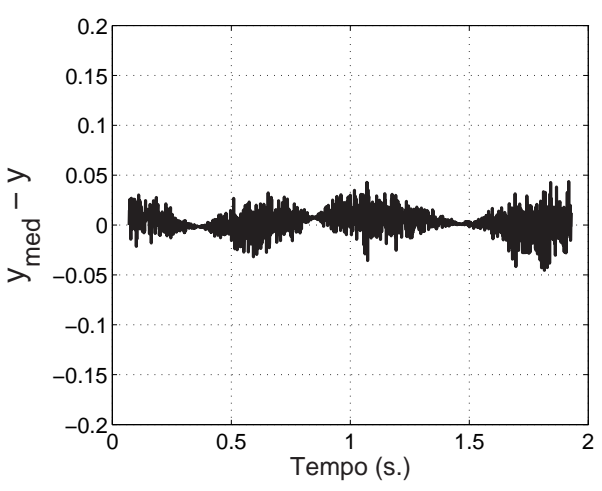

Figura 5.8: Diferença entre as saídas do sistema real e sistema auxiliar.

\subsection{Estimação dos Parâmetros Elétricos do Ge- rador}

A fim de estimar os parâmetros elétricos do gerador satisfazendo os requisitos práticos previamente apresentados, são propostas várias alternativas. Estas alternativas avaliam principalmente a disponibilidade de medidas e a robustez da metodologia em relação aos valores iniciais dos parâmetros. A escolha das variáveis de entrada e saída, bem como as variáveis de estados do modelo, é fundamental para encontrar um modelo adequado para a estimação dos parâmetros elétricos do gerador.

\subsubsection{Estimação dos parâmetros elétricos utilizando o mo- delo original do gerador}

Neste caso, os parâmetros são estimados a partir do modelo original do gerador. Portanto, o sistema auxiliar é representado pelas equações (5.1)-(5.6). A metodologia de sensibilidade de trajetória na sua formulação tradicional (vide Seção 2.6.1) é utilizada como algoritmo de estimação.

As medidas necessárias são $\left(\delta_{r}(t), \omega_{r}(t) E_{f d r}(t)\right)$, das quais $u=E_{f d r}$ é selecio- 
nada como entrada e $y_{\text {med }}=\left(\delta_{r}(t), \omega_{r}(t)\right)$ é selecionada como saída. Utiliza-se o sub-índice "r" para indicar que a variável ou medida pertence ao sistema real. O vetor de parâmetros é: $p=\left(x_{d}, x_{d}^{\prime}, T_{d o}^{\prime}, x_{q}, x_{q}^{\prime}, T_{q o}^{\prime}, E_{q o}^{\prime}, E_{d o}^{\prime}\right)$.

As equações de sensibilidade são obtidas derivando-se as equações (5.1)-(5.6) em relação a cada parâmetro. No diagrama de blocos da Figura 5.9, mostra-se a aplicação com esta alternativa.

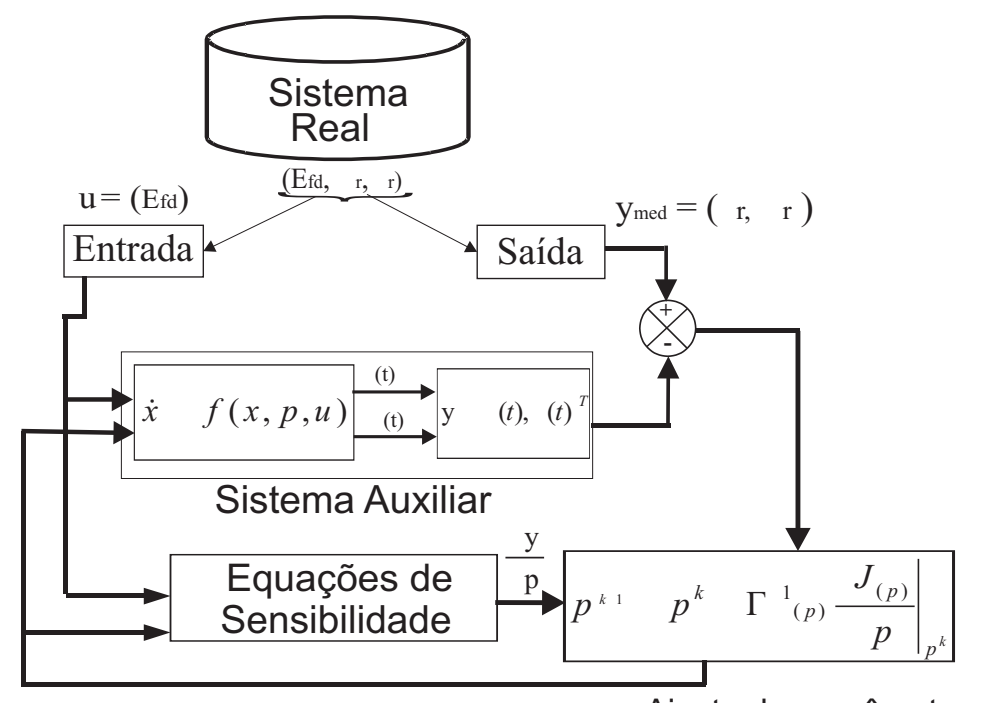

Ajuste dos parâmetros

Figura 5.9: Estimação de parâmetros elétricos utilizando o modelo original do gerador síncrono.

A metodologia conseguiu estimar os parâmetros mesmo com alterações entre $-15 \%$ e $+15 \%$ em relação aos valores verdadeiros. Este valor de $15 \%$ foi obtido aumentando a incerteza nos parâmetros gradativamente e verificando se a metodologia convergia próximo aos valores verdadeiros. Todos os parâmetros foram alterados em uma direção para satisfazer as restrições físicas $x_{d}^{\prime}<x_{q}^{\prime}<x_{q}<x_{d}$ e $T_{q o}^{\prime}<T_{d o}^{\prime}$. Na Tabela 5.3, mostram-se os resultados da estimação dos parâmetros elétricos utilizando esta alternativa.

Embora os parâmetros do gerador possam ser estimados com esta alternativa, a disponibilidade da medida $\delta(t)$, difícil obtenção em sistemas reais, e o conhecimento dos parâmetros da rede $\left(E_{B}\right.$ e $\left.Z_{I}\right)$ são necessários. Além disso, na prática, é muito difícil a obtenção de valores iniciais de $E_{q o}^{\prime}$ e $E_{d o}^{\prime}$ com incerteza inferiores a $\pm 15 \%$. Tudo isto limita a aplicação desta abordagem. 
Tabela 5.3: Estimação dos parâmetros elétricos do gerador utilizando o modelagem original.

\begin{tabular}{|c|c|c|c|c|c|}
\hline Parâmetro & $\begin{array}{c}\text { Estimativa } \\
\text { inicial }\end{array}$ & Desvio & $\begin{array}{c}\text { Estimativa } \\
\text { final }\end{array}$ & $\begin{array}{c}\text { Valor } \\
\text { final }\end{array}$ & $\begin{array}{c}\text { Erro } \\
(\%)\end{array}$ \\
\hline$x_{d}$ & 2,082 & $+15 \%$ & 1,810 & 1,810 & 0,000 \\
\hline$x_{d}^{\prime}$ & 0,345 & $+15 \%$ & 0,300 & 0,300 & 0,000 \\
\hline$T_{d o}^{\prime}$ & 9,200 & $+15 \%$ & 8,000 & 8,000 & 0,000 \\
\hline$x_{q}$ & 2,024 & $+15 \%$ & 1,760 & 1,760 & 0,000 \\
\hline$x_{q}^{\prime}$ & 0,747 & $+15 \%$ & 0,6500 & 0,650 & 0,000 \\
\hline$T_{q o}^{\prime}$ & 1,150 & $+15 \%$ & 1,000 & 1,000 & 0,000 \\
\hline$E_{q o}^{\prime}$ & 1,341 & $+15 \%$ & 1,1662 & 1,1662 & 0,000 \\
\hline$E_{d o}^{\prime}$ & $-0,357$ & $+15 \%$ & $-0,311$ & $-0,311$ & 0,000 \\
\hline
\end{tabular}

A fim de aumentar esta região de convergência, a próxima alternativa utiliza o acoplamente mestre-escravo para estimar os parâmetros elétricos.

\subsubsection{Estimação dos parâmetros elétricos utilizando o mo- delo original do gerador e com acoplamento mestre- escravo}

Nesta alternativa, repete-se o teste da Seção 5.5.1, porém acoplando o sistema real diretamente com o sistema auxiliar por intermédio da medida $\delta_{r}(t)$. As equações do sistema auxiliar são as mesmas do modelo original do gerador (5.1)(5.4) mudando apenas $\delta(t)$ por $\delta_{r}(t)$ nas equações (5.5)-(5.6), como é mostrado a seguir.

$$
\begin{aligned}
I_{d} & =\frac{E_{B} \cos \left(\delta_{r}\right)-E_{q}^{\prime}}{x_{d}^{\prime}+Z_{I}}, \\
I_{q} & =\frac{E_{B} \operatorname{sen}\left(\delta_{r}\right)+E_{d}^{\prime}}{x_{q}^{\prime}+Z_{I}} .
\end{aligned}
$$

As equações de sensibilidade são obtidas derivando-se as equações (5.1)-(5.4) em relação a cada parâmetro. A entrada de sincronização $\delta_{r}$ modifica as equações 
de sensibilidade quando comparada com a abordagem anterior. No diagrama de blocos da Figura $\underline{5.10}$ mostra-se a aplicação com esta alternativa. O acoplamento inserido nesta abordagem é explicitado com a linha tracejada.

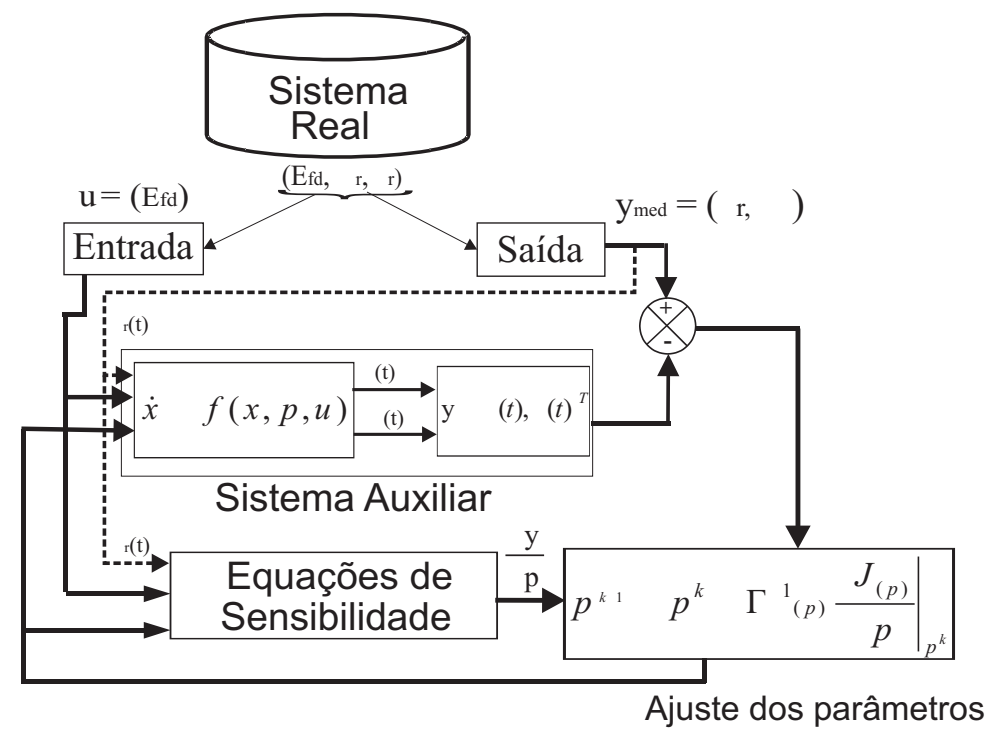

Figura 5.10: Estimação de parâmetros elétricos usando o modelo tradicional do gerador síncrono com acoplamento mestre-escravo.

As estimativas inicias para $E_{q o}^{\prime}$ e $E_{d o}^{\prime}$ são obtidas por intermédio das seguintes relações da máquina síncrona (Padiyar, 1996):

$$
\begin{aligned}
V_{d o} & =-V_{T o} \operatorname{sen}\left(\delta_{o}-\theta_{o}\right) \\
V_{q o} & =V_{T o} \cos \left(\delta_{o}-\theta_{o}\right) ; \\
I_{d o} & =-I_{T o} \operatorname{sen}\left(\delta_{o}-\phi_{o}\right) \\
I_{q o} & =I_{T o} \cos \left(\delta_{o}-\phi_{o}\right) ; \\
E_{q o}^{\prime} & =V_{q o}-x_{d}^{\prime} I_{d o} \\
E_{d o}^{\prime} & =V_{d o}+x_{q}^{\prime} I_{q o} .
\end{aligned}
$$

onde todas as variáveis e medidas estão referidas aos valores em regime permanente e $\delta_{o}$ é o valor inicial da medida aferida $\delta(t)$.

A metodologia conseguiu estimar os parâmetros mesmo com alterações entre $-20 \%$ e $+20 \%$ em relação aos valores verdadeiros. Considerando $+20 \%$ de alteração nos parâmetros, as estimativas iniciais para $E_{q o}^{\prime}$ e $E_{d o}^{\prime}$ usando (5.12) foram calculadas como é mostrado a seguir: 
Os dados em regime permanente são: $V_{T o}=1,00 ; \theta_{o}=9.63^{\circ} ; I_{T o}=1,121$; $\phi_{T o}=-26.95^{\circ} ; x_{d}^{\prime}=0,36(+20 \%$ do seu valor nominal $) ; x_{q}^{\prime}=0,78(+20 \%$ do seu valor nominal); e no início da amostragem $\delta_{o}=52,85$.

$$
\begin{aligned}
& V_{d o}=-1,00 \operatorname{sen}(52,85-9,63)=-0,685 \\
& V_{q o}=1,00 \cos (52,85-9,63)=0,729 ; \\
& I_{d o}=-1,121 \operatorname{sen}(52,85+26,95)=-1,103 \\
& I_{q o}=1,121 \cos (52,85+26,95)=0,198 ; \\
& E_{q o}^{\prime}=0,729-(0,36)(-1,103)=1,126 ; \\
& E_{d o}^{\prime}=-0,685+(0,78)(0,198)=-0,530 .
\end{aligned}
$$

As estimativas iniciais para $E_{q o}^{\prime}$ e $E_{d o}^{\prime}$, calculadas por este procedimento, estão em $-3,4 \%$ e $+70,4 \%$ (respectivamente) de seus valores corretos.

Os resultados da estimação dos parâmetros com esta abordagem são mostrados na Tabela 5.4. A região de convergência foi estimada em $\pm 20 \%$.

Tabela 5.4: Estimação de parâmetros da máquina síncrona para a opção 2.

\begin{tabular}{|c|c|c|c|c|c|}
\hline parâmetro & $\begin{array}{c}\text { Estimativa } \\
\text { Inicial }\end{array}$ & Desvio & $\begin{array}{c}\text { Estimativa } \\
\text { Final }\end{array}$ & $\begin{array}{c}\text { Valor } \\
\text { Final }\end{array}$ & $\begin{array}{c}\text { Erro } \\
(\%)\end{array}$ \\
\hline$x_{d}$ & 2,172 & $+20 \%$ & 1,810 & 1,8100 & 0,000 \\
\hline$x_{d}^{\prime}$ & 0,360 & $+20 \%$ & 0,300 & 0,300 & 0,000 \\
\hline$T_{d o}^{\prime}$ & 9,600 & $+20 \%$ & 8,00 & 8,000 & 0,000 \\
\hline$x_{q}$ & 2,112 & $+20 \%$ & 1,759 & 1,760 & $-0,056$ \\
\hline$x_{q}^{\prime}$ & 0,780 & $+20 \%$ & 0,650 & 0,6500 & 0,000 \\
\hline$T_{q o}^{\prime}$ & 1,200 & $+20 \%$ & 1,000 & 1,000 & 0,000 \\
\hline$E_{q o}^{\prime}$ & 1,126 & $-3,4 \%$ & 1,1662 & 1,1662 & 0,000 \\
\hline$E_{d o}^{\prime}$ & $-0,530$ & $+70,4 \%$ & $-0,311$ & $-0,311$ & 0,000 \\
\hline
\end{tabular}

A vantagens da abordagem apresentada nesta seção comparada com a abordagem da Seção 5.5 .1 é que a região de convergência aumentou de $\pm 15 \%$ para $\pm 20 \%$. Entretanto, uma atenção especial deve ser dada às estimativas iniciais de $E_{q o}^{\prime}$ e $E_{d o}^{\prime}$, pois o sucesso do processo de estimação depende das estimativas destes 
parâmetros não estejam distantes dos valores verdadeiros. As estimativas para $E_{q o}^{\prime}$ e $E_{d o}^{\prime}$ obtidas através das equações (5.12) não são muito boas, pois dependem de parâmetros que são incertos $\left(x_{d}^{\prime}\right.$ e $\left.x_{q}^{\prime}\right)$ e das correntes e tensões calculadas em regime permanente, fatos que comprometem a aplicação da metodologia de estimação utilizando esta abordagem.

\subsubsection{Estimação dos parâmetros elétricos utilizando o mo- delo do gerador modificado}

As principais limitações das alternativas apresentadas nas seções (5.5.1) e (5.5.2) é que estas necessitam: (i) da medida do ângulo do rotor $\delta(t)$, que é de difícil obtenção na prática ${ }^{2}$; (ii) dos parâmetros da rede; e (iii) de valores iniciais dos parâmetros próximos dos valores verdadeiros, em especial de $E_{q o}^{\prime}$ e $E_{d o}^{\prime}$.

Nesta proposta o gerador é modelado de tal forma que utilize somente medidas de fácil acesso. Para este objetivo a escolha das entradas e das saídas é fundamental. O primeiro passo foi mudar a referência de ângulo de fase. A modelagem tradicional usa a referência síncrona como referência para medida de ângulo (equações (5.1)-(5.6)). Entretanto, isto faz com que as equações do gerador dependam dos parâmetros da rede. Colocando a referência de ângulo nos terminais do gerador, as equações do gerador ficam independentes dos parâmetros da rede. Na Figura 5.11 estão apresentados os sistema de referência para medida do ângulo do rotor na referência síncrona $I_{m}-R_{e}$ (usada pelo modelo tradicional), na tensão terminal $u-v$ (proposta) e no eixo $d-q$.

Além disso, ao invés de utilizar $\delta$ e $\omega$ como saída, utiliza-se as variáveis $P_{e}$ e $Q_{e}$ que podem ser calculadas pelas seguintes equações:

$$
\begin{aligned}
P_{e} & =E_{d}^{\prime} I_{d}+E_{q}^{\prime} I_{q}+\left(x_{d}^{\prime}-x_{q}^{\prime}\right) I_{d} I_{q} \\
Q_{e} & =E_{d}^{\prime} I_{q}-E_{q}^{\prime} I_{d}-x_{q}^{\prime} I_{q}^{2}-x_{d}^{\prime} I_{d}^{2}
\end{aligned}
$$

\footnotetext{
${ }^{2}$ Uma alternativa para obter o ângulo de rotor é usar um APMU (Jin et al., 2007) que é um PMU especial. Entretanto, isto aumenta o custo da estimação.
} 


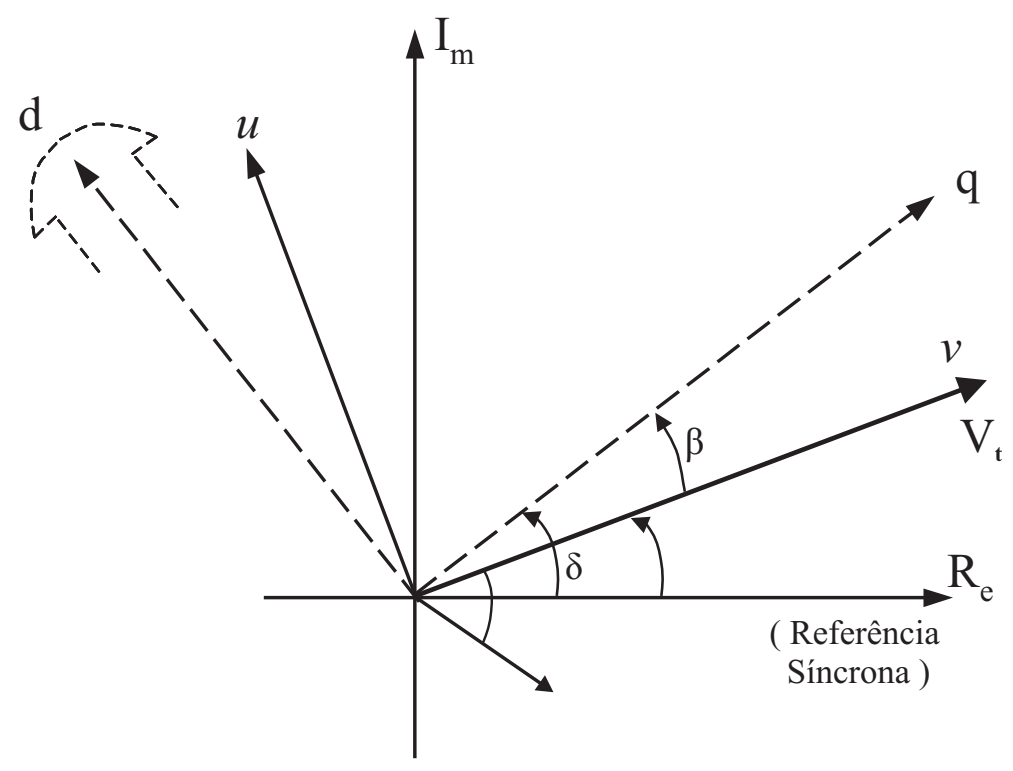

Figura 5.11: Sistema de referência da máquina síncrona (d-q), do sistema de potência $\left(I_{m}-R_{e}\right)$ e a proposta ( $u$-v, na tensão terminal).

Com esta abordagem, as equações da máquina síncrona estão composta por (5.3)(5.4), (5.13)-(5.14) e pelas seguintes equações:

$$
\begin{aligned}
\dot{\beta} & =\omega-\omega_{T}, \\
I_{d} & =-I_{T} \operatorname{sen}(\beta+\phi), \\
I_{q} & =I_{T} \cos (\beta+\phi),
\end{aligned}
$$

em que $\beta(t)$ é o ângulo do rotor na nova referência $u-v$, o qual coincide com o ângulo de potência, $\omega_{T}(t)$ é a freqüência de oscilação da tensão na barra terminal, e $\phi(t)$ é o fator de potência.

Para estimar os parâmetros as seguintes medidas são necessárias: $\left(V_{T}(t), I_{T}(t), \omega_{r}(t), \omega_{T r}(t), E_{f d}(t)\right)$, onde as tensões e correntes devem ser medidas nas três fases. A partir destas medidas, $P_{e}, Q_{e}$ e $\phi(t)$ podem ser facilmente calculados. As entradas para o modelo são as magnitudes da tensão e corrente nos terminais da máquina $\left(V_{T}\right.$ e $\left.I_{T}\right)$, a tensão de excitação, o fator de potência e a diferença entre a freqüência angular do rotor e a freqüência no terminal da máquina $\left(\omega-\omega_{T}\right)$.

O algoritmo de estimação baseado na sensibilidade de trajetória na sua formulação tradicional (vide Seção 2.6.1) é utilizado para estimar os parâmetros do 
gerador. As equações de sensibilidade são obtidas derivando-se as equações do gerador em relação a cada parâmetro. No diagrama de blocos da Figura 5.12 são mostradas as entradas e as saídas utilizadas nesta abordagem.

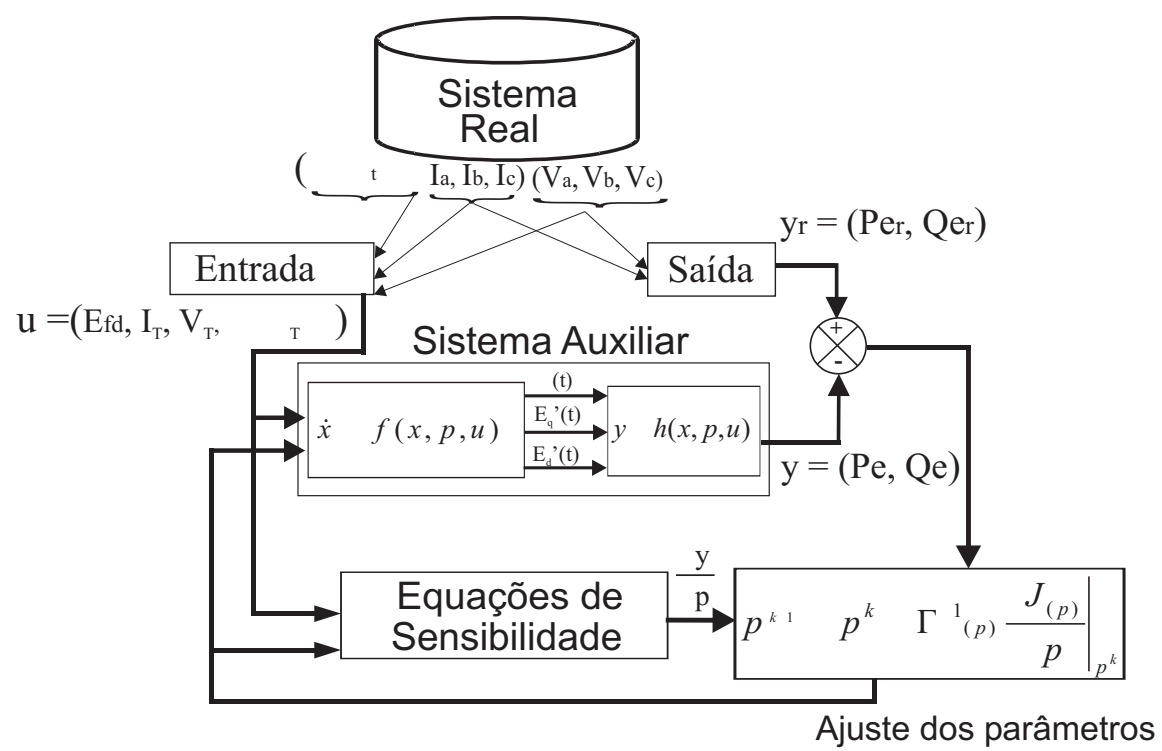

Figura 5.12: Diagrama de blocos para a estimação dos parâmetros com o modelo do gerador modificado.

Neste caso as estimativas para as tensões transitória de eixo direto e em quadratura foram assumidas os valores da máquina em vazio, isto é: $E_{q o}^{\prime}=V_{t o}$, $E_{d o}^{\prime}=0$. Esta forma de calcular a estimativa inicial para $E_{q o}^{\prime}$ e $E_{d o}^{\prime}$ é mais conveniente do que as apresentadas nas Seções (5.5.1) e (5.5.2), por serem mais gerais.

A metodologia conseguiu estimar os parâmetros mesmo com alterações entre $-80 \%$ e $+80 \%$ em relação aos valores verdadeiros (exceto $E_{q o}^{\prime}$ e $E_{d o}^{\prime}$ cujos valores iniciais são os da máquina em vazio, como foi mencionado anteriormente).

Como exemplo, na Tabela 5.5 são mostrados os resultados para uma alteração nos valores dos parâmetros de $+80 \%$ em relação aos valores verdadeiros.

Embora os testes computacionais tenham comprovado a estimação correta e ampla região de convergência com esta abordagem, o cálculo do ângulo de potência depende de $\omega(t)-\omega_{T}$ a qual é difícil de se medir com precisão, desde que $\omega(t)$ é muito próxima de $\omega_{T}(t)$. Isto, em geral, torna complicada a aplicação desta 
Tabela 5.5: Estimação de parâmetros da máquina síncrona para a opção 3.

\begin{tabular}{|c|c|c|c|c|c|}
\hline Parâmetro & $\begin{array}{c}\text { Estimativa } \\
\text { Inicial }\end{array}$ & Desvio & $\begin{array}{c}\text { Estimativa } \\
\text { Final }\end{array}$ & $\begin{array}{c}\text { Valor } \\
\text { verdadeiro }\end{array}$ & $\begin{array}{c}\text { Erro } \\
(\%)\end{array}$ \\
\hline$x_{d}$ & 3,258 & $+80 \%$ & 1,811 & 1,8100 & 0,110 \\
\hline$x_{d}^{\prime}$ & 0,5400 & $+80 \%$ & 0,299 & 0,300 & $-0,333$ \\
\hline$T_{d o}^{\prime}$ & 14,40 & $+80 \%$ & 8,091 & 8,000 & 1,137 \\
\hline$x_{q}$ & 3,168 & $+80 \%$ & 1,735 & 1,7600 & $-1,420$ \\
\hline$x_{q}^{\prime}$ & 1,170 & $+80 \%$ & 0,656 & 0,6500 & 0,923 \\
\hline$T_{q o}^{\prime}$ & 1,800 & $+80 \%$ & 0,9560 & 1,000 & $-4,400$ \\
\hline$E_{q o}^{\prime}$ & $V_{t o}=1,000$ & $-14,25 \%$ & 1,1662 & 1,1662 & 0,000 \\
\hline$E_{d o}^{\prime}$ & 0,000 & $-100 \%$ & $-0,307$ & $-0,311$ & $-0,124$ \\
\hline
\end{tabular}

abordagem em sistemas reais.

A fim de contornar esta dificuldade, na seqüência, propõe-se uma alternativa que estima os parâmetros sem a necessidade da medição do ângulo de potência.

\subsubsection{Estimação dos parâmetros elétricos utilizando o mo- delo do gerador modificado e estimando numerica- mente o ângulo de potência}

A abordagem anterior dependia do ângulo $\beta$, o qual é muito difícil de ser medido na prática. A partir da velocidade mecânica do rotor pode-se estimar $\delta$ via integração numérica de (5.1) conhecendo $\delta_{o}$ (o qual é difícil de ser obtido). Entretanto, esta informação não é suficiente para calcular $\beta$. Este pode ser calculado pela diferença de $\delta$ com o ângulo da tensão terminal $\theta$ (que pode ser obtido usando um PMU). Uma alternativa para medir $\delta$ diretamente é utilizar um tipo especial de medidor APMU, proposto em (Jin et al., 2007); no entanto, o uso de tal dispositivo em SEP ainda é muito limitado e caro.

Nesta alternativa, propõe-se um método para calcular numericamente $\beta$. Para 
este fim, considere a seguinte equação algébrica:

$$
I_{T}^{2}-I_{d}^{2}-I_{q}^{2}=0
$$

em que $I_{d}=\frac{V_{T} \cos (\beta)-E_{q}^{\prime}}{x_{d}^{\prime}}$ e $I_{q}=\frac{V_{T} \operatorname{sen}(\beta)+E_{d}^{\prime}}{x_{q}^{\prime}}$. A equação (5.18) relaciona a tensão e corrente nos terminais do gerador, as tensões internas $E_{d}^{\prime}$ e $E_{q}^{\prime}$, e os parâmetros $x_{d}^{\prime}$ e $x_{q} \operatorname{com} \beta$. As equações do modelo do gerador que constituirão o sistema auxiliar são as equações (5.3)-(5.4), a equação algébrica (5.18) e as saídas $P_{e}\left(\right.$ (5.13) e $Q_{e}$ (5.14). Para fins didáticos, estas são re-escritas na seqüência:

$$
\begin{aligned}
\dot{E}_{q}^{\prime} & =\frac{1}{T_{d o}^{\prime}}\left[E_{f d}-E_{q}^{\prime}+\left(x_{d}-x_{d}^{\prime}\right) I_{d}\right] \\
\dot{E}_{d}^{\prime} & =\frac{-1}{T_{q o}^{\prime}}\left[E_{d}^{\prime}+\left(x_{q}-x_{q}^{\prime}\right) I_{q}\right] \\
0 & =I_{T}^{2}-I_{d}^{2}-I_{q}^{2} \\
P_{e} & =E_{d}^{\prime} I_{d}+E_{q}^{\prime} I_{q}+\left(x_{d}^{\prime}-x_{q}^{\prime}\right) I_{d} I_{q} \\
Q_{e} & =E_{d}^{\prime} I_{q}-E_{q}^{\prime} I_{d}-x_{q}^{\prime} I_{q}^{2}-x_{d}^{\prime} I_{d}^{2} \\
I_{d} & =\frac{V_{T} \cos (\beta)-E_{q}^{\prime}}{x_{d}^{\prime}} \\
I_{q} & =\frac{V_{T} \operatorname{sen}(\beta)+E_{d}^{\prime}}{x_{q}^{\prime}}
\end{aligned}
$$

em que $I_{d}$ e $I_{q}$ são variáveis intermediárias. Este modelo contém oito parâmetros $p=\left(x_{d}, x_{d}^{\prime}, T_{d o}^{\prime}, x_{q}, x_{q}^{\prime}, T_{q o}^{\prime}, E_{q o}^{\prime}, E_{d o}^{\prime}\right)^{T}$, duas variáveis de estado $x=\left(E_{q}^{\prime}(t), E_{d}^{\prime}(t)\right)$ e uma variável algébrica $z=(\beta(t))$. O vetor de entrada é $u=\left(E_{f d}(t), I_{t}(T), V_{t}(T)\right)$ e o vetor de saída é $y=\left(P_{e}(t), Q_{e}(t)\right)$.

Os parâmetros deste modelo podem ser estimados a partir das EADs do Sistema auxiliar (5.19)-(5.25) utilizando a metodologia de estimação estudada na Seção 2.6. Além disso, o ângulo de potência $\beta(t)$ pode ser calculado pela integração numérica de (5.19)-(5.21).

Na seqüência, será avaliado o desempenho dos algoritmos de ajuste de sensibilidade de trajetória com a formulação tradicional (Seção 2.6.1), sensibilidade de trajetória com a abordagem de minimização (Seção 2.6.2) e, ao final, a combinação destas abordagens com o algoritmo de estimação em duas fases (Seção 2.7). 


\section{A) Algoritmo de sensibilidade de trajetória tradicional}

Nesta abordagem, a solução do sistema auxiliar e as funções de sensibilidade devem ser calculadas utilizando um método numérico de integração. A regra trapezoidal foi escolhida para este objetivo.

\section{Solução do sistema auxiliar}

Considere as EADs (5.19)-(5.21) do sistema auxiliar e aplicando a regra trapezoidal, obtém-se:

$$
\begin{aligned}
E_{q(n+1)}^{\prime}-\frac{\Delta t}{2}\left[\frac{1}{T_{d o}^{\prime}}\left(E_{f d(n+1)}-E_{q(n+1)}^{\prime}+\left(x_{d}-x_{d}^{\prime}\right) I_{d(n+1)}\right)\right]-E_{q(n)}^{\prime} & \cdots \\
-\frac{\Delta t}{2}\left[\frac{1}{T_{d o}^{\prime}}\left(E_{f d(n)}-E_{q(n)}^{\prime}+\left(x_{d}-x_{d}^{\prime}\right) I_{d(n)}\right)\right] & =0, \\
E_{d(n+1)}^{\prime}-\frac{\Delta t}{2}\left[\frac{-1}{T_{q o}^{\prime}}\left(E_{d(n+1)}^{\prime}+\left(x_{q}-x_{q}^{\prime}\right) I_{q(n+1)}\right)\right]-E_{d(n)}^{\prime} & \cdots \\
-\frac{\Delta t}{2}\left[\frac{-1}{T_{q o}^{\prime}}\left(E_{d(n)}^{\prime}+\left(x_{q}-x_{q}^{\prime}\right) I_{q(n)}\right)\right] & =0, \\
I_{T(n+1)}^{2}-I_{d(n+1)}^{2}-I_{q(n+1)}^{2} & =0,
\end{aligned}
$$

onde os sub-índices $n$ e $n+1$ indicam os instantes de tempo $t_{n}=t_{o}+n \Delta t$ e $t_{n+1}=t_{o}+(n+1) \Delta t$, respectivamente.

Este conjunto de equações não-lineares pode ser resolvido utilizando o método de Newton nas variáveis $E_{q(n+1)}^{\prime}, E_{d(n+1)}^{\prime}, \beta_{(n+1)}$. Para tanto, utiliza-se como estimativa inicial $E_{q(n+1)}^{\prime}=E_{q(n)}^{\prime}, E_{d(n+1)}^{\prime}=E_{d(n)}^{\prime}$ e $\beta_{(n+1)}=\beta_{(n)}$.

Denotando as equações $(5.26),(5.27)$ e (5.28) por $F 1, G 1$ e $H 1$, respectivamente, o vetor que contém estas equações é dado por:

$$
W 1=\left(\begin{array}{c}
F 1\left(E_{q(n+1)}^{\prime}, E_{d(n+1)}^{\prime}, \beta_{(n+1)}\right) \\
G 1\left(E_{q(n+1)}^{\prime}, E_{d(n+1)}^{\prime}, \beta_{(n+1)}\right) \\
H 1\left(E_{q(n+1)}^{\prime}, E_{d(n+1)}^{\prime}, \beta_{(n+1)}\right)
\end{array}\right)
$$

Fazendo $V_{v}=\left(E_{q(n+1)}^{\prime}, E_{d(n+1)}^{\prime}, \beta_{(n+1)}\right)$ e aplicando o método de Newton, na iteração $k$ obtém-se:

$$
V_{v}^{k+1}=V_{v}^{k}-\left.\left(\frac{\partial W 1}{\partial V_{v}}\right)^{-1} W 1\right|_{k}
$$


onde $\frac{\partial W 1}{\partial V_{v}}$ é a matriz Jacobiana, dada por:

$$
\frac{\partial W 1}{\partial V_{v}}=\left(\begin{array}{ccc}
\frac{\partial F 1}{\partial E_{q(n+1)}^{\prime}} & \frac{\partial F 1}{\partial E_{d(n+1)}^{\prime}} & \frac{\partial F 1}{\partial \beta_{(n+1)}} \\
\frac{\partial G 1}{\partial E_{q(n+1)}^{\prime}} & \frac{\partial G 1}{\partial E_{d(n+1)}^{\prime}} & \frac{\partial G 1}{\partial \beta_{(n+1)}} \\
\frac{\partial H 1}{\partial E_{q(n+1)}^{\prime}} & \frac{\partial H 1}{\partial E_{d(n+1)}^{\prime}} & \frac{\partial H 1}{\partial \beta_{(n+1)}}
\end{array}\right)
$$

Este procedimento é repetido em todo o período de amostragem (vide algoritmo da Seção 2.5.2). No instante inicial $n=0$, as seguintes estimativas foram consideradas $E_{q o}^{\prime}=V_{t o}, E_{d o}^{\prime}=0$ e $\beta_{o}=\tan ^{-1} \frac{P_{e}}{V_{t}^{2} / x_{d}+Q_{e}}$.

\section{Equações de sensibilidade}

As equações de sensibilidade são obtidas derivando-se (5.19)-(5.23) em relação a cada parâmetro. Para o parâmetro $x_{d}$, por exemplo, tem-se:

$$
\begin{aligned}
\dot{\lambda}_{E_{q}^{\prime}}^{x_{d}} & =\frac{1}{T_{d o}^{\prime}}\left(-\lambda_{E_{q}^{\prime}}^{x_{d}}+I_{d}+\left(x_{d}-x_{d}^{\prime}\right) \frac{\partial I_{d}}{\partial x_{d}}\right), \\
\dot{\lambda}_{E_{d}^{\prime}}^{x_{d}} & =\frac{-1}{T_{q o}^{\prime}}\left(\lambda_{E_{d}^{\prime}}^{x_{d}}+\left(x_{q}-x_{q}^{\prime}\right) \frac{\partial I_{q}}{\partial x_{d}}\right), \\
0 & =-2 I_{d} \frac{\partial I_{d}}{\partial x_{d}}-2 I_{q} \frac{\partial I_{q}}{\partial x_{d}}, \\
\lambda_{P_{e}}^{x_{d}} & =\lambda_{E_{d}^{\prime}}^{x_{d}} I_{d}+E_{d}^{\prime} \frac{\partial I_{d}}{\partial x_{d}}+\lambda_{E_{q}^{\prime}}^{x_{d}} I_{q}+E_{q}^{\prime} \frac{\partial I_{q}}{\partial x_{d}}+\left(x_{d}^{\prime}-x_{q}^{\prime}\right)\left(\frac{\partial I_{d}}{\partial x_{d}} I_{q}+I_{d} \frac{\partial I_{q}}{\partial x_{d}}\right), \\
\lambda_{Q_{e}}^{x_{d}} & =\lambda_{E_{d}^{\prime}}^{x_{d}} I_{q}+E_{d}^{\prime} \frac{\partial I_{q}}{\partial x_{d}}-\lambda_{E_{q}^{\prime}}^{x_{d}} I_{d}-E_{q}^{\prime} \frac{\partial I_{d}}{\partial x_{d}}-2 x_{q}^{\prime} I_{q} \frac{\partial I q}{\partial x_{d}}-2 x_{d}^{\prime} I_{d} \frac{\partial I d}{\partial x_{d}},
\end{aligned}
$$

onde $\frac{\partial I_{d}}{\partial x_{d}}=\frac{1}{x_{d}^{\prime}}\left(-V_{T} \operatorname{sen}(\beta) \lambda_{\beta}^{x_{d}}-\lambda_{E_{q}^{\prime}}^{x_{d}}\right)$ e $\frac{\partial I_{q}}{\partial x_{d}}=\frac{1}{x_{q}^{\prime}}\left(V_{T} \cos (\beta) \lambda_{\beta}^{x_{d}}+\lambda_{E_{d}^{\prime}}^{x_{d}}\right)$.

As equações de sensibilidade (5.32)-(5.34) formam um conjunto de EADs que pode ser resolvido utilizando a regra trapezoidal como método de integração numérica da mesma forma que o sistema auxiliar. No final do tempo amostrado, as funções de sensibilidades $\lambda_{E_{q}^{\prime}}^{x_{d}}, \lambda_{E_{d}^{\prime}}^{x_{d}}$ e $\lambda_{\beta}^{x_{d}}$ serão determinadas. Substituindo estas nas equações (5.35)-(5.36) as sensibilidades do vetor de saída são calculadas. As sensibilidades em relação aos outros parâmetros podem ser encontradas repetindose o mesmo procedimento.

A Figura 5.13 apresenta o diagrama de blocos do processo de estimação dos parâmetros do gerador por intermédio desta abordagem. A entrada está composta pelas amplitudes de tensões e correntes trifásicas e pela tensão de excitação. A saída é composta pelas potência ativa e reativa. O sistema auxiliar é representado 
pelas EADs (5.19)-(5.25) e resolvido utilizando integração numérica. A matriz $\Gamma$ e o vetor $\frac{\partial J(p)}{\partial p}$ são determinados a partir das funções de sensibilidade e das equações $(2.25)-(2.27)$.

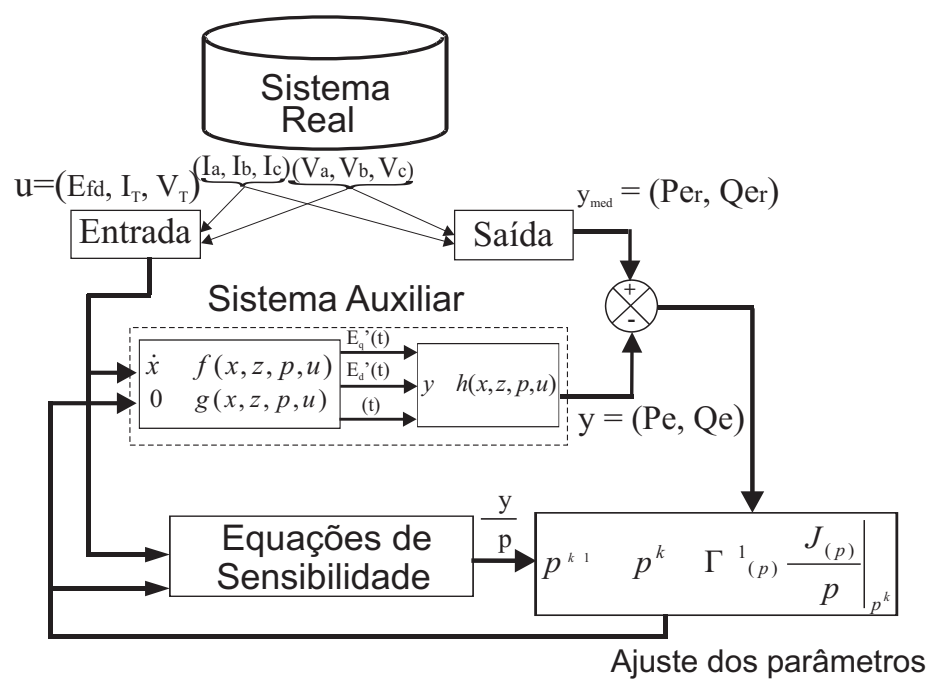

Figura 5.13: Estimação de parâmetros elétricos com a metodologia de sensibilidade de trajetória na formulação tradicional.

\section{Resultados}

Quando todos os parâmetros são estimados simultaneamente, a metodologia de sensibilidade de trajetória tradicional só consegue estimar os parâmetros com alterações entre $-2 \%$ e $+2 \%$ em relação aos valores verdadeiros (incluindo os parâmetros $E_{q o}^{\prime}$ e $E_{d o}^{\prime}$ ). Para maiores incertezas do que $\pm 2 \%$ o algoritmo de ajuste dos parâmetros produz um conjunto de parâmetros para os quais a equação algébrica não tem solução (singularidades) e a estimação não pode ser efetuada. Desta forma, não é possível a aplicação desta abordagem na práticas. Além disso, garantir que os valores inicial de $E_{q o}^{\prime}$ e $E_{d o}^{\prime}$ estejam no máximo $\pm 2 \%$ distanciados dos seus valores verdadeiros é muito difícil. Considerando estimativas mais gerais para estes parâmetros de $E_{q o}^{\prime}=V_{t o}$ e $E_{d o}^{\prime}=0$, verificou-se que a metodologia não consegue estimar os parâmetros mesmo colocando os valores iniciais dos outros parâmetros em seus valores verdadeiros.

Para contornar os problemas de singularidades na equação algébrica, apresenta- 
se a seguir a aplicação do algoritmo de sensibilidade de trajetória com abordagem de minimização, estudada na Seção (2.6.2), para a solução das EADs do gerador síncrono.

\section{B) Algoritmo de sensibilidade de trajetória com abordagem de mini- mização}

Os problemas de solvabilidade da equação algébrica do sistema de EADs do sistema auxiliar podem ser evitados substituindo-se as equações (5.19)-(5.23) pelo problema de minimização conforme proposto na Seção 2.6 .2 .

\section{Solução do sistema auxiliar}

O modelo de minimização do Sistema auxiliar é:

$$
\begin{aligned}
& \min _{(x, z)} \quad g^{2}\left(E_{q}^{\prime}, E_{d}^{\prime}, \beta, p, I, V_{t}, E_{f d}\right) \\
& \text { s.a. }\left\{\begin{array}{rrr}
\dot{E}_{q}^{\prime} & = & \frac{1}{T_{d o}^{\prime}}\left[E_{f d}-E_{q}^{\prime}+\left(x_{d}-x_{d}^{\prime}\right) I_{d}\right], \\
\dot{E}_{d}^{\prime} & = & \frac{-1}{T_{q o}^{\prime}}\left[E_{d}^{\prime}+\left(x_{q}-x_{q}^{\prime}\right) I_{q}\right],
\end{array}\right. \\
& y=\left\{\begin{array}{l}
P_{e}=E_{d}^{\prime} I_{d}+E_{q}^{\prime} I_{q}+\left(x_{d}^{\prime}-x_{q}^{\prime}\right) I_{d} I_{q}, \\
Q_{e}=E_{d}^{\prime} I_{q}-E_{q}^{\prime} I_{d}-x_{q}^{\prime} I_{q}^{2}-x_{d}^{\prime} I_{d}^{2},
\end{array}\right.
\end{aligned}
$$

sendo $g(\overbrace{E_{q}^{\prime}, E_{d}^{\prime}}^{x}, \overbrace{\beta}^{z}, p, \overbrace{I_{T}, V_{T}, E_{f d}}^{u})=I_{T}^{2}-I_{d}^{2}-I_{q}^{2}$. O vetor de saída $y$ pode ser calculado por simples substituição das variáveis $E_{q}^{\prime}(t), E_{d}^{\prime}(t)$ e $\beta(t)$ e não será considerado no equacionamento nas seções seguintes.

Usando a regra trapezoidal para integrar as equações diferenciais, a forma discreta do problema prévio é dada por:

$$
\begin{gathered}
\min _{(x, z)} g_{(n+1)}^{2}\left(E_{q}^{\prime}, E_{d}^{\prime}, \beta, p, I_{t}, V_{t}, E_{f d}\right) \\
\text { s.a }\left\{\begin{array}{l}
E_{q(n+1)}^{\prime}-E_{q(n)}^{\prime}-\frac{\Delta t}{2} f_{1(n+1)}-\frac{\Delta t}{2} f_{1(n)}=0 \\
E_{d(n+1)}^{\prime}-E_{d(n)}^{\prime}-\frac{\Delta t}{2} f_{2(n+1)}-\frac{\Delta t}{2} f_{2(n)}=0
\end{array}\right.
\end{gathered}
$$

sendo:

$$
\begin{aligned}
& f_{1}=\frac{1}{T_{d o}^{\prime}}\left(E_{f d}-E_{q}^{\prime}+\left(x_{d}-x_{d}^{\prime}\right) I_{d}\right) \\
& f_{2}=-\frac{1}{T_{q o}^{\prime}}\left(E_{d}^{\prime}+\left(x_{q}-x_{q}^{\prime}\right) I_{q}\right)
\end{aligned}
$$


Os sub-índices $n$ e $n+1$ denotam os valores das variáveis nos tempos $t_{n}=t_{o}+n \Delta t$ e $t_{n+1}=t_{o}+(n+1) \Delta t$, respectivamente.

A função Lagrangeana associada é dada por

$$
\begin{aligned}
L= & g_{(n+1)}^{2}+\lambda_{L 1}\left(E_{q(n+1)}^{\prime}-E_{q(n)}^{\prime}-\frac{\Delta t}{2} f_{1(n+1)}-\frac{\Delta t}{2} f_{1(n)}\right) \\
& +\lambda_{L 2}\left(E_{d(n+1)}^{\prime}-E_{d(n)}^{\prime}-\frac{\Delta t}{2} f_{2(n+1)}-\frac{\Delta t}{2} f_{2(n)}\right)
\end{aligned}
$$

onde $\lambda_{L 1}$ e $\lambda_{L 2}$ são os multiplicadores de Lagrange.

Aplicando a primeira condição de otimalidade de KKT (Karush-Kuhn-Tucker) (Bazaraa, 1979) em (5.41), tem-se que:

$$
\frac{\partial L}{\partial E_{q(n+1)}^{\prime}}=2 g_{(n+1)} \frac{\partial g_{(n+1)}}{\partial E_{q(n+1)}^{\prime}}+\lambda_{L 1}\left(1-\frac{\Delta t}{2} \frac{\partial f_{1(n+1)}}{\partial E_{q(n+1)}^{\prime}}\right)+\lambda_{L 2}\left(-\frac{\Delta t}{2} \frac{\partial f_{2(n+1)}}{\partial E_{q(n+1)}^{\prime}}\right)
$$

sendo

$$
\begin{aligned}
\frac{\partial g_{(n+1)}}{\partial E_{q(n+1)}^{\prime}} & =-2 I_{d(n+1)} \frac{\partial I_{d(n+1)}}{\partial E_{q(n+1)}^{\prime}}-2 I_{q(n+1)} \frac{\partial I_{q(n+1)}}{\partial E_{q(n+1)}^{\prime}} \\
\frac{\partial I_{d(n+1)}}{\partial E_{q(n+1)}^{\prime}} & =-\frac{1}{x_{d}^{\prime}} \\
\frac{\partial I_{q(n+1)}}{\partial E_{q(n+1)}^{\prime}} & =0 \\
\frac{\partial f_{1(n+1)}}{\partial E_{q(n+1)}^{\prime}} & =\frac{1}{T_{d o}^{\prime}}\left[-1+\left(x_{d}-x_{d}^{\prime}\right) \frac{\partial I_{d(n+1)}}{\partial E_{q(n+1)}^{\prime}}\right]=\frac{1}{T_{d o}^{\prime}}\left[-1-\frac{\left(x_{d}-x_{d}^{\prime}\right)}{x_{d}^{\prime}}\right], \\
\frac{\partial f_{2(n+1)}}{\partial E_{q(n+1)}^{\prime}} & =\frac{-1}{T_{q o}^{\prime}}\left[\left(x_{q}-x_{q}^{\prime}\right) \frac{\partial I_{q(n+1)}}{\partial E_{q(n+1)}^{\prime}}\right]=0 .
\end{aligned}
$$

Substituindo (5.44)-(5.47) em (5.42) tem-se (5.48):

$$
\frac{\partial L}{\partial E_{q(n+1)}^{\prime}}=4 g_{(n+1)} \frac{I_{d(n+1)}}{x_{d}^{\prime}}+\lambda_{L 1}\left[1+\frac{\Delta t}{2 T_{d o}^{\prime}}\left(1+\frac{x_{d}-x_{d}^{\prime}}{x_{d}^{\prime}}\right)\right] .
$$

Procedendo de maneira similar encontra-se $\frac{\partial L}{\partial E_{d(n+1)}^{\prime}}, \frac{\partial L}{\partial \beta_{n+1}}, \frac{\partial L}{\partial \lambda_{L 1(n+1)}}$ e $\frac{\partial L}{\partial \lambda_{L 2(n+1)}}$. As equações obtidas derivando a função Lagrangeana $L$ pelas variáveis $E_{q(n+1)}^{\prime}$, $E_{d(n+1)}^{\prime}, \beta_{(n+1)}, \lambda_{L 1(n+1)}$ e $\lambda_{L 2(n+1)}$ é: 


$$
\begin{aligned}
\frac{\partial L}{\partial E_{q(n+1)}^{\prime}}= & 4 g_{(n+1)} \frac{I_{d(n+1)}}{x_{d}^{\prime}}+\lambda_{L 1}\left[1+\frac{\Delta t}{2 T_{d o}^{\prime}}\left(1+\frac{x_{d}-x_{d}^{\prime}}{x_{d}^{\prime}}\right)\right] \\
\frac{\partial L}{\partial E_{d(n+1)}^{\prime}}= & -4 g_{(n+1)} \frac{I_{q(n+1)}}{x_{q}^{\prime}}+\lambda_{L 2}\left[1+\frac{\Delta t}{2 T_{q o}^{\prime}}\left(1+\frac{x_{q}-x_{q}^{\prime}}{x_{q}^{\prime}}\right)\right] \\
\frac{\partial L}{\partial \beta_{(n+1)}}= & 2 g_{(n+1)}\left(\frac{2 I_{d(n+1)}}{x_{d}^{\prime}} V_{t(n+1)} \operatorname{sen}\left(\beta_{n+1}\right)-\frac{2 I_{q(n+1)}}{x_{q}^{\prime}} V_{t(n+1)} \cos \left(\beta_{n+1)}\right)+\ldots\right. \\
\frac{\partial L}{\partial \lambda_{L 1(n+1)}=} & \lambda_{L 1}\left(\frac{\Delta t}{2} \frac{x_{d(n+1)}-x_{d}^{\prime}}{x_{d}^{\prime}} V_{t(n+1)} \operatorname{sen}\left(\beta_{n+1}\right)\right)+\lambda_{L 2}\left(\frac{\Delta t}{2} \frac{x_{q}-x_{q}^{\prime}}{x_{q}^{\prime}} V_{t(n+1)} \cos \left(\beta_{n+1}\right)\right) \\
& \frac{\Delta t}{2 T_{q o}^{\prime}}\left(E_{f d(n)}-E_{q(n)}^{\prime}+\left(x_{d o}-x_{f d(n+1)}-E_{q(n+1)}^{\prime}+\left(x_{d}-x_{d}^{\prime}\right) I_{d(n+1)}\right)-\ldots\right. \\
\frac{\partial L}{\partial \lambda_{L 2(n+1)}}= & E_{d(n+1)}^{\prime}-E_{d(n)}^{\prime}+\frac{\Delta t}{2 T_{q o}^{\prime}}\left(E_{d(n+1)}^{\prime}+\left(x_{q}-x_{q}^{\prime}\right) I_{q(n+1)}\right)+ \\
& \frac{\Delta t}{2 T_{q o}^{\prime}}\left(E_{d(n)}^{\prime}+\left(x_{q}-x_{q}^{\prime}\right) I_{q(n)}\right)
\end{aligned}
$$

Sendo $I_{d(n)}=\frac{V_{T(t)} \cos \left(\beta_{n}\right)-E_{q(n)}^{\prime}}{x_{d}^{\prime}}, I_{d(n+1)}=\frac{V_{T(n+1)} \cos \left(\beta_{(n+1)}\right)-E_{q(n+1)}^{\prime}}{x_{d}^{\prime}}, I_{q(n)}=\frac{V_{T(n)} \operatorname{sen}\left(\beta_{n}\right)+E_{d(n)}^{\prime}}{x_{q}^{\prime}}$, $I_{q(n+1)}=\frac{V_{T(n+1)} \operatorname{sen}\left(\beta_{(n+1)}\right)+E_{d(n+1)}^{\prime}}{x_{q}^{\prime}}$ e $g_{(n+1)}=I_{T(n+1)}-I_{d(n+1)}-I_{q(n+1)}$.

Este conjunto de equações não-lineares pode ser resolvido pelo método de Newton nas variáveis $E_{q(n+1)}^{\prime}, E_{d(n+1)}^{\prime}, \beta_{(n+1)}, \lambda_{L 1(n+1)}$ e $\lambda_{L 2(n+1)}$. Para isto utiliza-se como estimativa inicial $E_{q(n+1)}^{\prime}=E_{q(n)}^{\prime}, E_{d(n+1)}^{\prime}=E_{d(n)}^{\prime}, \beta_{(n+1)}=\beta_{(n)}$, $\lambda_{L 1(n+1)}=\lambda_{L 1(n)}$ e $\lambda_{L 2(n+1)}=\lambda_{L 2(n)}$.

Definindo:

$$
\begin{aligned}
W 1 & =\left(\frac{\partial L}{\partial E_{q(n+1)}^{\prime}}, \frac{\partial L}{\partial E_{d(n+1)}^{\prime}}, \frac{\partial L}{\partial \beta_{(n+1)}}, \frac{\partial L}{\partial \lambda_{L 1(n+1)}}, \frac{\partial L}{\partial \lambda_{L 2(n+1)}}\right)^{T}, \\
V_{v} & =\left(E_{q(n+1)}^{\prime}, E_{d(n+1)}^{\prime}, \beta_{(n+1)}, \lambda_{L 1(n+1)}, \lambda_{L 2(n+1)}\right)^{T},
\end{aligned}
$$

e aplicando o método de Newton para a iteração $k$, obtém-se:

$$
V_{v}^{k+1}=V_{v}^{k}-\left.\left(\frac{\partial W 1}{\partial V_{v}}\right)^{-1} W 1\right|_{k}
$$

Este procedimento deve ser repetido para todo $n$ dentro do período de integração. Ao longo do processo serão determinadas as variáveis do sistema auxiliar: $E_{q}^{\prime}(t), E_{d}^{\prime}(t), \beta(t), \lambda_{L 1}(t)$ e $\lambda_{L 2}(t)$ (vide procedimento para integrar equações algébrico-diferencias da Seção (2.5.3)).

No instante inicial $n=0$, as seguintes estimativas foram utilizadas: $E_{q o}^{\prime}=V_{t o}, E_{d o}^{\prime}=0$ e $\beta_{o}=\tan ^{-1} \frac{P_{e}}{V_{T}^{2} / x_{d}+Q_{e}}$.

No apêndice $\mathrm{B}$ mostra-se as equações da matriz Jacobiana $\frac{\partial W 1}{\partial V_{v}}$. 


\section{Equações de Sensibilidade}

As equações de sensibilidade de trajetória podem ser obtidas derivando-se as equações (5.49)-(5.53) em relação a cada parâmetro e a sua solução pode ser obtida usando o método de Newton.

A Figura 5.14 apresenta o diagrama de blocos com a abordagem de minimização proposta nesta Seção. O sistema auxiliar é modelado pelo problema de minimização (5.37)-(5.38). A matriz $\Gamma$ e o vetor $\frac{\partial J(p)}{\partial p}$ são determinados a partir das funções de sensibilidade e das equações (2.25)-(2.27).

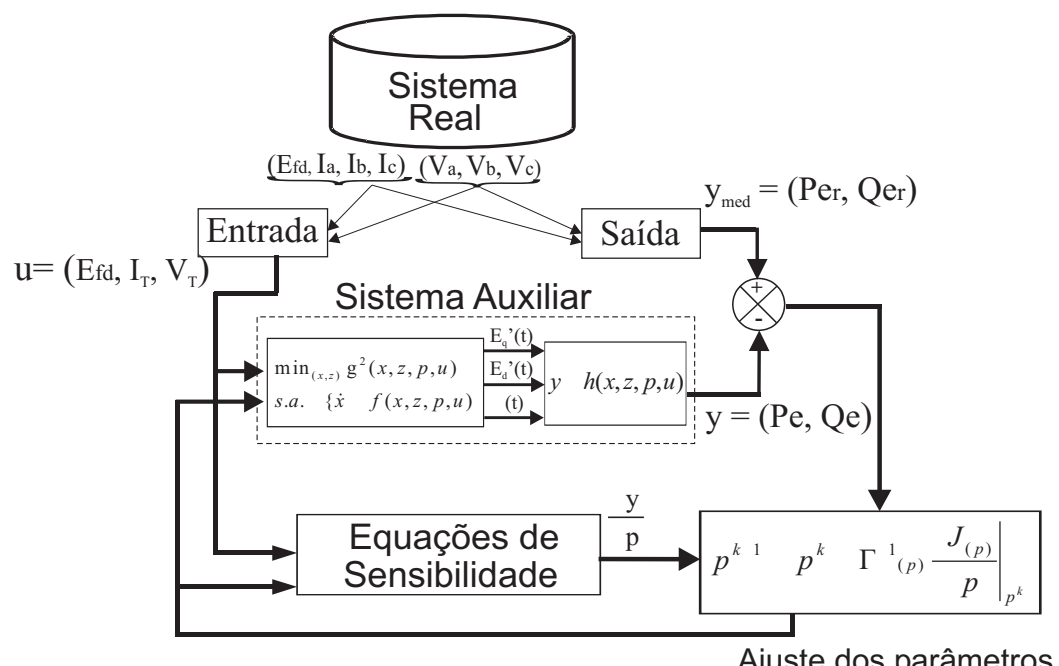

Figura 5.14: Estimação de parâmetros com a metodologia de sensibilidade de trajetória com a abordagem de minimização.

As equações para encontrar as funções de sensibilidade estão mostradas no Apêndice B.

\section{Resultados}

Os resultados mostram que a metodologia com esta abordagem garante a convergência aos valores verdadeiros se os valores iniciais dos parâmetros estiverem distantes dos valores nominais na faixa que vai desde $-28 \%$ até $+6 \%$ de erro. Isto indica uma melhora na região de convergência quando comparada com a metodologia de sensibilidade de trajetória na sua formulação tradicional apresentada na Seção 5.5.4. 
A Figura $\left[5.15\right.$ compara a região de convergência para os parâmetros $x_{d}^{\prime}$ e $x_{q}^{\prime}$ (os outros parâmetros são considerados corretos). A linha sólida mostra a fronteira da região de convergência da abordagem de minimização proposta, enquanto que a linha tracejada representa a fronteira da região de convergência da metodologia de sensibilidade de trajetória tradicional. Como pode observar-se, a abordagem de minimização aumenta a região de convergência, isto é, os parâmetros convergem aos valores verdadeiros para erros maiores nos valores iniciais. Os benefícios da abordagem de minimização tornam-se mais evidentes a medida que se aumenta o número de parâmetros a serem estimados simultaneamente

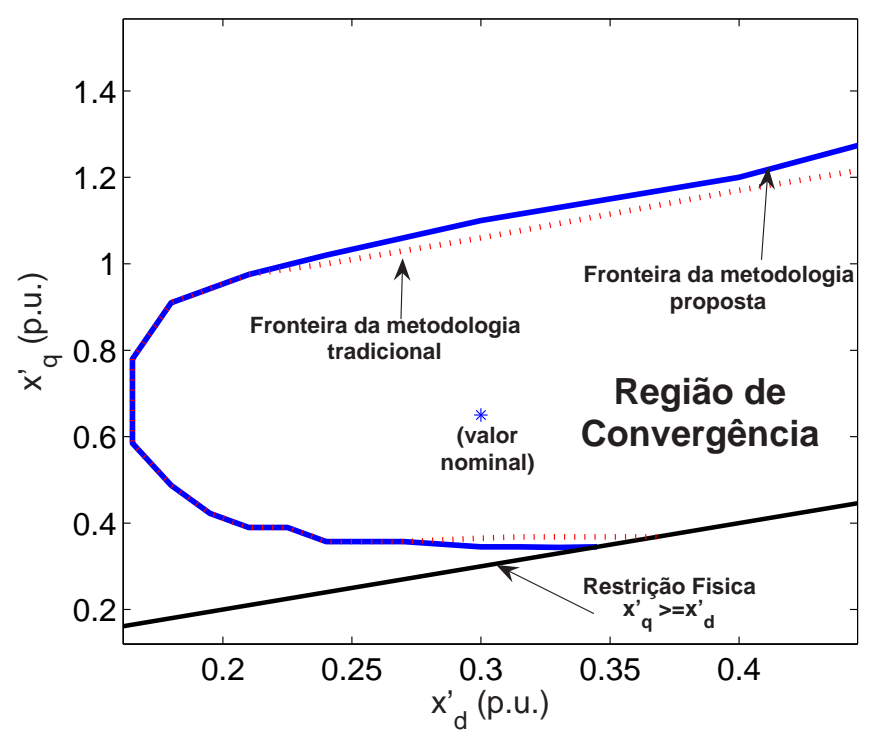

Figura 5.15: Comparação da região de convergência para a metodologia de sensibilidade de trajetória tradicional e com a abordagem de minimização para os parâmetros $x_{d}^{\prime}$ e $x_{q}^{\prime}$.

Embora a região de convergência tenha aumentado com a abordagem de minimização, ainda é pequena para aplicações práticas. A fim de se obter uma região de convergência satisfatória, os algoritmos de estimação apresentados anteriormente serão combinados com o algoritmo de estimação de duas fases estudado na Seção 2.7 . 
C) Combinação da metodologia de estimação com o algoritmo de estimação em duas fases

A fim de se estimar os parâmetros em duas fases, os parâmetros serão classificados segundo sua influência na saída utilizando a decomposição $Q R$ (Burth et al., 1999), estudada na Seção (2.7.1).

\section{Classificação dos parâmetros segundo sua influência na saída}

A matriz $\Gamma$ foi gerada a partir do sistema auxiliar modelado por minimização (5.37)-(5.38), considerando a estimativa inicial do vetor de parâmetros elétricos distanciada em $-35 \%$ do valor verdadeiro, $E_{q o}^{\prime}=V_{t o}$ e $E_{d o}^{\prime}=0$ de acordo com a Figura (5.16).

\begin{tabular}{rl|r|r|r|r|r|r|}
\multicolumn{1}{c}{$x_{d}$} & \multicolumn{1}{c}{$x_{d}^{\prime}$} & \multicolumn{1}{c}{$T_{d o}^{\prime}$} & \multicolumn{1}{c}{$x_{q}$} & \multicolumn{1}{c}{$x_{q}^{\prime}$} & \multicolumn{1}{c}{$T_{q o}^{\prime}$} & \multicolumn{1}{c|}{$E_{q o}^{\prime}$} & \multicolumn{1}{c|}{$E^{\prime}{ }_{d o}$} \\
\hline 1,9852 & 4,0225 & 0,1704 & $-0,0749$ & 0,7348 & $-0,1469$ & $-5,1553$ & $-0,3042$ \\
\hline 4,0225 & 22,9258 & 0,4535 & $-0,9587$ & 0,1375 & 0,1841 & $-17,1562$ & 0,3386 \\
\hline 0,1704 & 0,4535 & 0,017 & $-0,0081$ & 0,0564 & $-0,0112$ & $-0,445$ & $-0,0246$ \\
\hline$-0,0749$ & $-0,9587$ & $-0,0081$ & 0,0831 & 0,0043 & $-0,0403$ & 0,7716 & $-0,0495$ \\
\hline 0,7348 & 0,1375 & 0,0564 & 0,0043 & 0,5403 & $-0,082$ & $-1,2193$ & $-0,2732$ \\
\hline$-0,1469$ & 0,1841 & $-0,0112$ & $-0,0403$ & $-0,082$ & 0,0473 & $-0,0101$ & 0,0804 \\
\hline$-5,1553$ & $-17,1562$ & $-0,445$ & 0,7716 & $-1,2193$ & $-0,0101$ & 19,4787 & $-0,1426$ \\
\hline$-0,3042$ & 0,3386 & $-0,0246$ & $-0,0495$ & $-0,2732$ & 0,0804 & $-0,1426$ & 0,2359 \\
\hline
\end{tabular}

Figura 5.16: Matriz $\Gamma$ obtida a partir das equações da máquina síncrona. Na parte superior é possível observar a ordem original dos parâmetros.

Aplicando o algoritmo da Seção (2.7.1), os parâmetros foram ordenados segundo sua influência na saída. Os resultados estão apresentados na Tabela 5.6, juntamente com os valores singulares da Matriz $\Gamma$. De acordo com os valores singulares observados, os parâmetros que mais influenciam a saída (parâmetros bemcondicionados) são $E_{q o}^{\prime}$ e $x_{d}^{\prime}$; e os parâmetros com menor influência (parâmetros mal-condicionados) correspondem $T_{q o}^{\prime}, x_{q}$ e $T_{d o}^{\prime}$. 
Tabela 5.6: Classificação dos parâmetros segunda sua influência na saída.

\begin{tabular}{|c|c|c|c|c|c|c|c|c|}
\hline Par. & $x_{d}^{\prime}$ & $E_{q o}^{\prime}$ & $E_{d o}^{\prime}$ & $x_{d}$ & $x_{q}^{\prime}$ & $T_{q o}^{\prime}$ & $x_{q}$ & $T_{d o}^{\prime}$ \\
\hline V. Sing & 39,6 & 4,5 & 0,95 & 0,14 & 0,0198 & $57,1 \cdot 10^{-4}$ & $16 \cdot 10^{-4}$ & $1,3 \cdot 10^{-4}$ \\
\hline \multicolumn{1}{|c|}{ bem-condicionados } & $\longleftrightarrow$ & \multicolumn{3}{c|}{ mal-condicionados } \\
\hline
\end{tabular}

\section{Resultados}

De acordo com a análise previamente realizada, $T_{q o}^{\prime}, x_{q}$ e $T_{d o}^{\prime}$ são os parâmetros que menos influenciam a saída do modelo. Portanto, estes parâmetros foram mantidos constantes nas primeiras iterações, conforme o algoritmo de estimação em duas fases descrito na Seção (2.7). Os resultados mostram usando o algoritmo de estimação em duas fases com a metodologia de sensibilidade de trajetória tradicional, a metodologia conseguiu estimar os parâmetros mesmo com alterações entre $-29 \%$ e $+15 \%$ em relação aos valores verdadeiros. Por outro lado, estimando os parâmetros em duas fases com a metodologia que considera a abordagem de minimização, a metodologia conseguiu estimar os parâmetros mesmo com alterações entre $-35 \%$ e $+67 \%$ em relação aos valores verdadeiros.Em ambos os casos as estimativas iniciais para $E_{q o}^{\prime}$ e $E_{d o}^{\prime}$ foram $V_{t o}$ e zero, respectivamente. Claramente a maior região de convergência foi obtida combinando o algoritmo de estimação em duas fases com a metodologia de sensibilidade de trajetória que considera a abordagem de minimização.

A Tabela 5.7 mostra os resultados com a metodologia de sensibilidade de trajetória com a abordagem de minimização em duas fases. Na primeira fase, $T_{q o}^{\prime}$, $x_{q}$ e $T_{d o}^{\prime}$ foram mantidos fixos e os outros parâmetros foram atualizados até que $J(p)$ não mude de uma iteração para outra o que ocorreu depois de 6 iterações. Posteriormente, todos os parâmetros foram atualizados simultaneamente até a convergência depois de 5 iterações da metodologia.

As Figuras 5.17 e 5.18 apresentam os valores assumidos pela função $g^{2}($. no início e no final do processo de estimação. No início do processo, a função $g($.$) não possui solução no intervalo entre 1,7 e 2$ segundos (vide Figura 5.17) e a metodologia baseada em sensibilidade de trajetória tradicional não pode ser 
Tabela 5.7: Estimação em duas fases utilizando o algoritmo de sensibilidade de trajetória com abordagem de minimização.

\begin{tabular}{|c|c|c|c|c|c|c|}
\hline Parâmetro & $\begin{array}{c}\text { Estimativa } \\
\text { inicial }\end{array}$ & $\begin{array}{c}\text { Desvio } \\
(\%)\end{array}$ & $\begin{array}{c}\text { Fase } \\
1\end{array}$ & $\begin{array}{c}\text { Fase } \\
2\end{array}$ & $\begin{array}{c}\text { Valor } \\
\text { nominal }\end{array}$ & $\begin{array}{c}\text { Erro } \\
(\%)\end{array}$ \\
\hline$x_{d}$ & 1,21 & $-33 \%$ & 1,83 & 1,81 & 1,81 & 0,00 \\
\hline$x_{d}^{\prime}$ & 0,20 & $-33 \%$ & 0,35 & 0,30 & 0,30 & 0,00 \\
\hline$T_{d o}^{\prime}$ & 5,36 & $-33 \%$ & 5,36 & 7,99 & 8,00 & $-0,12$ \\
\hline$x_{q}$ & 1,18 & $-33 \%$ & 1,18 & 1,759 & 1,76 & $-0,06$ \\
\hline$x_{q}^{\prime}$ & 0,44 & $-33 \%$ & 1,15 & 0,65 & 0,65 & 0,00 \\
\hline$T_{q o}^{\prime}$ & 0,67 & $-33 \%$ & 0,67 & 0,996 & 1,00 & $-0,40$ \\
\hline$E_{q o}^{\prime}$ & $V_{T o}=1,00$ & $-14,25 \%$ & 1,28 & 1,166 & 1,166 & 0,00 \\
\hline$E_{d o}^{\prime}$ & 0,00 & $-100 \%$ & 0,19 & $-0,311$ & $-0,311$ & 0,00 \\
\hline
\end{tabular}

utilizada, enquanto que com a abordagem de minimização relaxa-se esta restrição, permitindo a aplicação da metodologia. Como esperado, a função $g($.$) é nula$ em todo o intervalo de integração depois da convergência dos parâmetros, como ilustrado na Figura 5.18 .

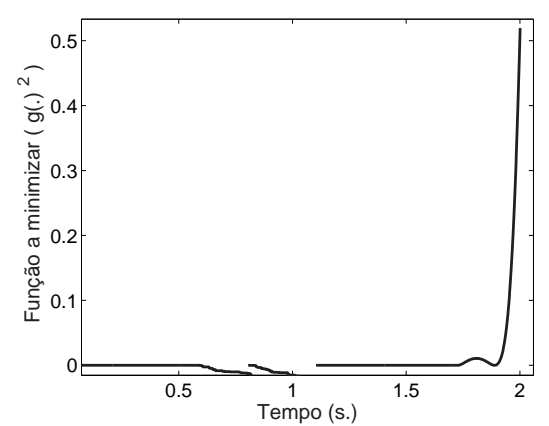

Figura 5.17: Função $g(.)^{2}$ no começo do processo de estimação.

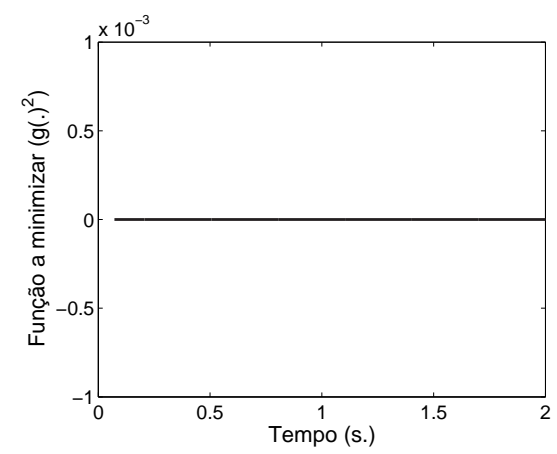

Figura 5.18: Função $g(.)^{2}$ no final do processo de estimação.

A evolução de $J(p)$ ao longo das iterações é ilustrada na Figura 5.19. Nesta Figura é possível observar que $J(p)$ tende a zero ao final das iterações, indicando que as saídas do sistema auxiliar e do sistema real se tornaram muito próximas. A Figura 5.20 apresenta o erro do ângulo de potência estimado ao final das iterações. 
Foram necessárias 11 iterações até a convergência final do processo de estimação, das quais 6 iterações foram realizadas na fase 1 e 5 iterações na fase 2 . O tempo de cálculo aproximado foi de 3 minutos em um computador pentium IV.

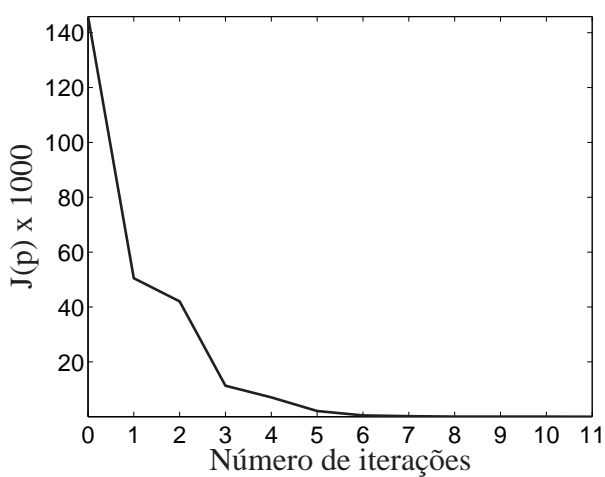

Figura 5.19: Evolução de $J(p)$ ao longo das iterações.

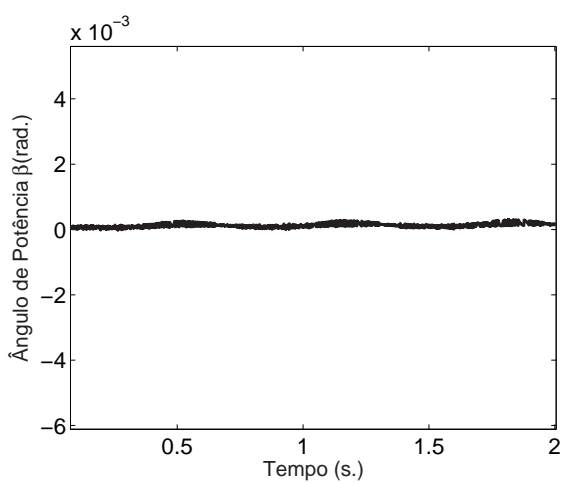

Figura 5.20: $\Delta \beta(t)$ após a convergência dos parâmetros.

\section{Influência do ruído das medidas na estimação dos parâmetros}

Nesta seção avalia-se a robustez da metodologia de sensibilidade de trajetória com abordagem de minimização frente ao ruído. Para tanto, foi adicionado ruído Gaussiano com média zero e desvio padrão de $1 \%$ em relação a seu valor nominal em todas as medidas e o teste anterior foi novamente repetido.

A metodologia conseguiu estimar os parâmetros mesmo com alterações entre $-32 \%$ e $+32 \%$ em relação aos valores verdadeiros.

A precisão de estimação foi afetada como mostra a Tabela 5.8. O maior erro de estimação encontrado foi de $10 \%$ no parâmetro $T_{q o}^{\prime}$. Uma alternativa para evitar a influência do ruído seria filtrar as medidas antes de serem utilizadas pela metodologia proposta.

Nas Figuras 5.21, 5.22 e 5.23, 5.24, comparam-se a potência ativa e reativa antes e depois da convergência dos parâmetros. Como pode ser observado, no início as saídas estão muito distantes, ao passo que quando os parâmetros convergem, as saídas ficam muito próximas. 
Tabela 5.8: Influência do ruído nas medidas na metodologia de sensibilidade de trajetória com abordagem de minimização e o algoritmo de estimação em duas fases.

\begin{tabular}{|c|c|c|c|c|c|c|}
\hline Parametro & $\begin{array}{c}\text { Estimativa } \\
\text { inicial }\end{array}$ & Desvio & $\begin{array}{c}\text { Fase } \\
1\end{array}$ & $\begin{array}{c}\text { Fase } \\
2\end{array}$ & $\begin{array}{c}\text { Valor } \\
\text { nominal }\end{array}$ & $\begin{array}{l}\text { Erro } \\
(\%)\end{array}$ \\
\hline$x_{d}$ & 2,39 & $+32 \%$ & 1,839 & 1,806 & 1,810 & $-0,22$ \\
\hline$x_{d}^{\prime}$ & 0,40 & $+32 \%$ & 0,351 & 0,303 & 0,300 & 1,00 \\
\hline$T_{d o}^{\prime}$ & 10,56 & $+32 \%$ & 5,360 & 7,993 & 8,000 & $-0,09$ \\
\hline$x_{q}$ & 2,32 & $+32 \%$ & 1,179 & 1,711 & 1,760 & $-2,78$ \\
\hline$x_{q}^{\prime}$ & 0,86 & $+32 \%$ & 1,038 & 0,669 & 0,650 & 2,92 \\
\hline$T_{q o}^{\prime}$ & 1,32 & $+32 \%$ & 0,670 & 0,898 & 1,000 & $-10,20$ \\
\hline$E_{q o}^{\prime}$ & $V_{t o}$ & $-13 \%$ & 1,285 & 1,171 & 1,166 & 0,43 \\
\hline$E_{d o}^{\prime}$ & 0,00 & $-100 \%$ & 0,148 & $-0,296$ & $-0,311$ & $-4,82$ \\
\hline
\end{tabular}

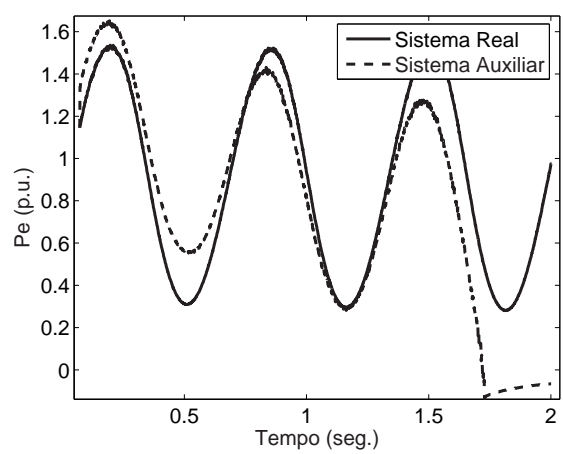

Figura 5.21: Potência ativa no início do processo de estimação com ruído nas medidas.

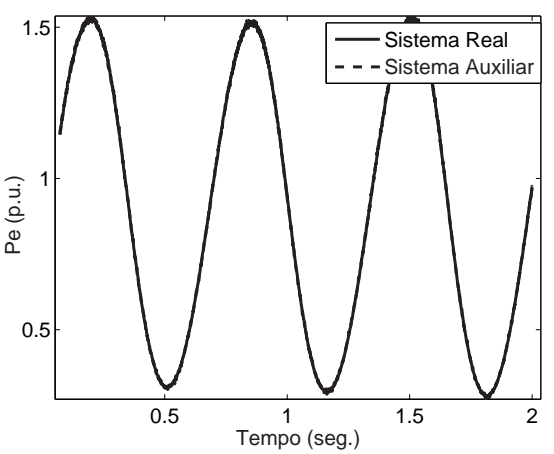

Figura 5.22: Potência ativa após a convergência dos parâmetros com ruído nas medidas.

Como subproduto da metodologia, o ângulo de potência $\beta(t)$ também é estimado. As Figuras 5.25 e 5.26 mostram o erro de estimação do ângulo de potência $(\Delta \beta)$. Como pode ser observado, o erro de estimação $\Delta \beta$ torna-se muito pequeno após a convergência dos parâmetros.

Finalmente, na Tabela 5.9 comparam-se as principais alternativas para estimar os parâmetros elétricos, das quais a alternativa 4 apresenta os melhores resultados porque não depende da medida do ângulo de potência e possui uma boa região 


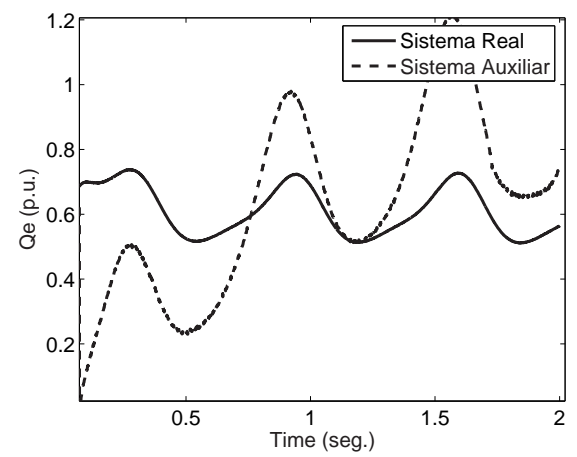

Figura 5.23: Potência reativa no início do processo de estimação com ruído nas medidas.

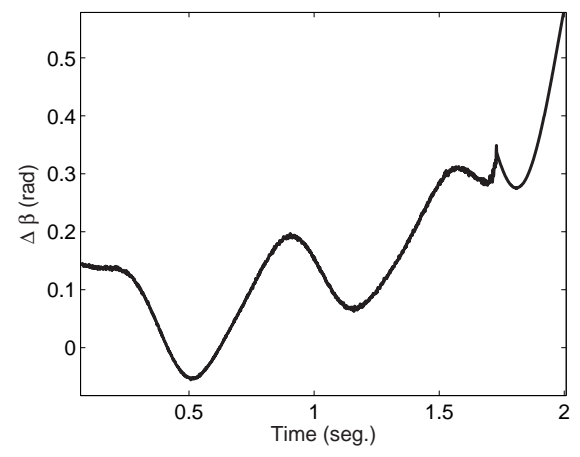

Figura 5.25: $\Delta \beta(t)$ entre os sistemas real e auxiliar no começo do processo de estimação.

de convergência.

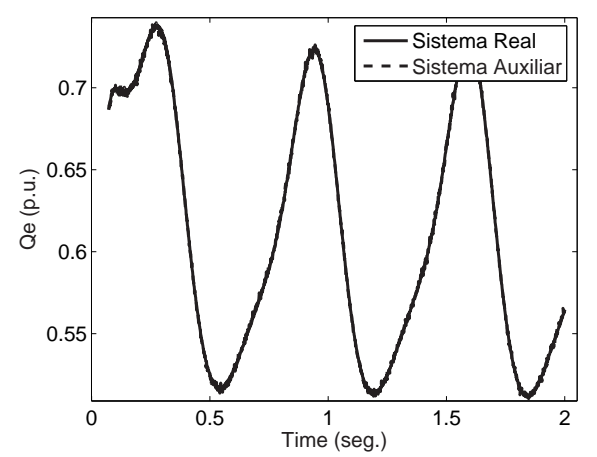

Figura 5.24: Potência reativa após a convergência dos parâmetros com ruído nas medidas.

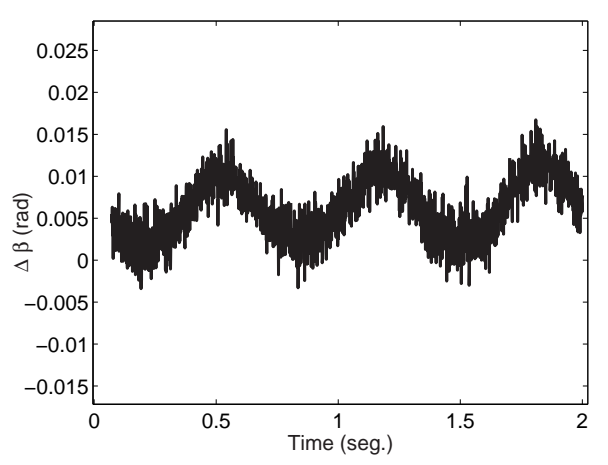

Figura 5.26: $\Delta \beta(t)$ entre os sistemas real e auxiliar no final do processo de estimação 


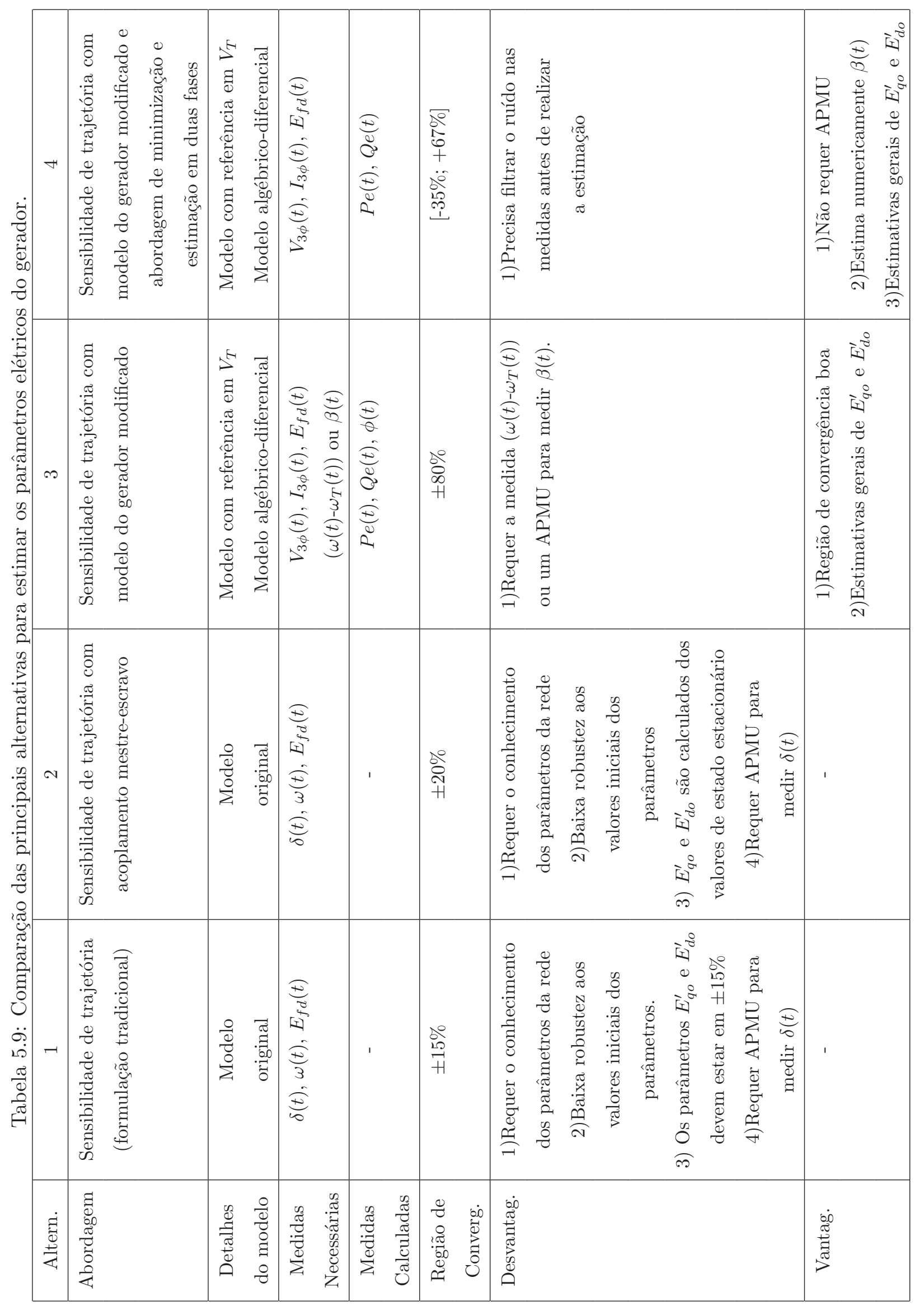




\section{Capítulo 6}

\section{Conclusões}

As principais contribuições foram: (i) proposição de uma nova metodologia de estimação de parâmetros baseada na análise de sensibilidade de trajetória de sistemas dinâmicos não-lineares restritos, ou seja, modelados por equações algébricodiferenciais; (ii) aplicação da metodologia proposta para estimação de parâmetros de geradores síncronos de tal forma a viabilizar a estimativa de parâmetros do mesmo satisfazendo uma série de requisitos práticos, tais como, obtenção de estimativas dos parâmetros a partir de medidas de perturbação obtidas com o gerador em operação, uso de medidas de fácil obtenção e robustez com relação aos valores iniciais dos parâmetros.

A metodologia de sensibilidade de trajetória foi utilizada como algoritmo de ajuste de parâmetros cujo objetivo é promover a sincronização das saídas do sistema real e do sistema auxiliar (modelo). Quando as saídas sincronizam, obtém-se um conjunto de parâmetros para o modelo escolhido que reproduz o comportamento dinâmico do sistema real. Entretanto, verificou-se que a formulação tradicional da metodologia de sensibilidade de trajetória apresenta dificuldades de convergência devido principalmente a: (i) impossibilidade de lidar com singularidades que podem se apresentar nas equações algébricas do modelo de EADs (equações algébrica-diferenciais) que levam à inexistência de soluções, especialmente quando os valores dos parâmetros estão distantes dos valores verdadeiros, (ii) baixa robustez com relação aos valores iniciais dos parâmetros e ruído nas medidas. Para contornar a primeira dificuldade propôs-se nesta tese uma nova 
metodologia, baseada na sensibilidade de trajetória para estimar parâmetros de sistemas dinâmicos não-lineares restritos, ou seja, modelado por EADs. Mais precisamente, relaxa-se a restrição de igualdade do sistema dinâmico, substituindo-a por uma formulação alternativa baseada na minimização da função algébrica do modelo de EADs. Esta nova formulação permite a estimativa de parâmetros mesmo quando as equações algébricas não possuem solução, aumentando significativamente a região de convergência da metodologia.

Para contornar os problemas de baixa robustez em relação a valores iniciais de parâmetros e ruído nas medidas, diversas técnicas foram estudadas. Dentre elas podem mencionar-se: (i) acoplamento mestre-escravo, e (ii) algoritmo de estimação em duas fases. Verificou-se através de alguns exemplos que a região de convergência aumenta após o uso do acoplamento principalmente quando este substitui termos não-lineares do sistema auxiliar. O uso deste tipo de acoplamento em conjunto com a metodologia de sensibilidade de trajetória seja uma contribuição inédita deste trabalho. O algoritmo de estimação em duas fases baseia-se na classificação dos parâmetros segundo suas sensibilidades. Este algoritmo é uma modificação do algoritmo proposto por (Burth et al., 1999), onde, na primeira fase os parâmetros com maior sensibilidade são atualizados pela metodologia em algumas iterações, e na segunda fase todos os parâmetros são atualizados simultaneamente até a convergência. Como resultado final, um algoritmo que combina a nova metodologia de sensibilidade de trajetória para sistemas restritos com o algoritmo de estimação em duas fases foi proposto para estimar parâmetros de modelos de geradores síncronos. Apesar de ter sido desenvolvida para resolver o problema de estimação de parâmetros do gerador síncrono, a metodologia é geral e pode ser aplicada para uma classe grande de sistemas dinâmicos não-lineares.

Uma contribuição importante desta tese está relacionada à modelagem do gerador. A escolha conveniente de variáveis de estado do modelo, das entradas e saídas, é fundamental para o sucesso da metodologia de estimação de parâmetros. Nesta tese, estas escolhas permitiram que os parâmetros mecânicos e elétricos pudessem ser estimados em forma desacoplada. 
Para estimar os parâmetros mecânicos, foi escolhido um modelo composto por uma equação diferencial que relaciona a velocidade mecânica, a potência ativa e potência mecânica com os parâmetros mecânicos. Escolhe-se a potência ativa e a potência mecânica como entrada e a velocidade de rotação mecânica como saída. Esta escolha de entradas e saídas é contribuição inédita desta pesquisa e permitiu que os parâmetros mecânicos fossem estimados com facilidade usando a metodologia de sensibilidade de trajetória tradicional. A metodologia também se mostrou robusta aos valores iniciais dos parâmetros e ruído nas medidas.

A estimação dos parâmetros elétricos é mais problemática. Verificou-se que a convergência da metodologia de estimação depende, em grande proporção, dos valores iniciais dos parâmetros, especialmente aqueles de variáveis não mensuráveis $\left(E_{q o}^{\prime}\right.$ e $\left.E_{d o}^{\prime}\right)$. Além do mais, o modelo do gerador, comumente usado para estimar os parâmetros elétricos (modelo usado para estudos de estabilidade transitória) possui o ângulo de rotor $\delta(t)$ como variável de estado. Esta variável é difícil de ser medida em grandes geradores e requer o uso de PMUs especiais. Além disso, o modelo tradicional do gerador depende dos parâmetros da rede. Ao aplicar a metodologia de sensibilidade de trajetória diretamente no modelo tradicional do gerador, verificou-se convergência da estimação somente quando os valores inicias dos parâmetros estavam muito próximos dos valores verdadeiros $( \pm 15 \%)$. Uma alternativa para aumentar a região de convergência $( \pm 20 \%)$ foi o emprego do acoplamento mestre-escravo com a metodologia de sensibilidade de trajetória. Mesmo assim, ainda persistiam as dificuldades do modelo original do gerador, ou seja, as estimativas dos parâmetros dependiam do conhecimento preciso das variáveis da rede e de medidas de difícil obtenção, tal como o ângulo do rotor.

Para contornar estas dificuldades, propôs-se um nova modelagem para o gerador, cujo objetivo era eliminar a dependência dos parâmetros da rede e da necessidade da obtenção de medidas especiais. O primeiro passo para atingir este objetivo foi colocar a referência para medida de ângulo nos terminais do gerador. Com isto, as equações do gerador ficam independentes dos parâmetros da rede. Além disso, escolhe-se a potência ativa $\left(P_{e}\right)$ e a potência reativa $\left(Q_{e}\right)$ como saídas 
e a corrente nos terminais do gerador $\left(I_{T}\right)$ e a diferença de freqüências entre o rotor e a tensão terminal $\left(\omega-\omega_{T}\right)$, como entradas. Com este novo modelo e usando a metodologia de sensibilidade de trajetória tradicional, os parâmetros podem ser estimados em forma robusta aos valores iniciais de parâmetros (região de convergência de $\pm 80 \%)$. Entretanto, o ângulo de potência depende de $\omega-\omega_{T}$ que é muito difícil de se medir com precisão em sistemas reais, o que inviabiliza esta abordagem na prática.

Para eliminar a necessidade de medir $\omega-\omega_{T}$, incorporou-se uma equação algébrica ao modelo do gerador que relaciona as variáveis de estado com o ângulo de potência. Assim, a máquina síncrona é modelada por um sistema algébricodiferencial. Entretanto, esta abordagem apresenta alguns problemas devido a inexistência de soluções das EADs. Isto acontece principalmente quando os parâmetros do sistema auxiliar estão distantes $\pm 2 \%$ de seus valores verdadeiros. Nesta situação, a equação algébrica deixa de ter solução e o procedimento de estimação não pode continuar (apresenta singularidades). Para evitar este problema, propôs-se o novo algoritmo de sensibilidade de trajetória com a abordagem de minimização. Isto aumentou a região de convergência e permitiu estimar os parâmetros considerando erros nos valores iniciais dos parâmetros na faixa que vai desde $-28 \%$ até $+6 \%$. Entretanto, estes resultados ainda limitavam sua aplicação na prática. Melhores resultados foram conseguidos combinando o algoritmo de estimação em duas fases com as metodologias de estimação desenvolvidas (sensibilidade de trajetória na formulação tradicional e com abordagem de minimização). No caso da metodologia baseada em sensibilidade de trajetória na formulação tradicional, a região de convergência aumentou do intervalo $(-2 \%$, $+2)$ para $(-29 \%,+26 \%)$. No caso da metodologia que utiliza a abordagem de minimização, a região de convergência aumentou do intervalo $(-28 \%,+6 \%)$ para $(-35 \%,+67 \%)$. Também foi avaliado a influência do ruído nas medidas para esta última alternativa. Após adicionar ruído Gaussiano com média zero e desvio padrão de $1 \%$ em relação a seu valor nominal. A região de convergência e a precisão dos parâmetros estimados foram afetados pelo ruído nas medidas. A 
região de convergência diminuiu de $(-35 \%,+67 \%)$ para $\pm 32 \%$ e o maior erro de estimação foi de $10 \%$ no parâmetro $T_{q o}^{\prime}$.

Como resultado final, foi viabilizada a estimação dos parâmetros do gerador síncrono com a metodologia de sensibilidade de trajetória, que até então tinha problemas de convergência e precisava de medidas de difícil obtenção.

Como um subproduto da modelagem do gerador proposto, o ângulo de potência foi simultaneamente estimado satisfatoriamente com os parâmetros durante todo o intervalo em análise .

\subsection{Considerações Finais e Perspectivas Futuras}

Devido à indisponibilidade de dados de perturbações reais, não foi possível aplicar a metodologia em um gerador síncrono em funcionamento. Entretanto, está aberta a possibilidade, caso alguma empresa disponibilize os dados de perturbações, de realizar isto no futuro. A continuação da presente pesquisa seria considerar um modelo mais detalhado (modelo sub-transitório), considerar falhas assimétricas (monofásica) e incluir o efeito da saturação no processo de estimação de parâmetros da máquina síncrona. Finalmente, outra importante aplicação da metodologia desenvolvida poderia ser a estimação de parâmetros de outros componentes de sistemas de potência, tais como modelos de cargas dinâmicas, que também estão modelados por EADs e estão sujeitos aos mesmos problemas de convergência que o modelo do gerador. 


\section{Bibliografia}

Aguirre, L. A. (1995). Controllability and observability of linear systems: Some noninvariant aspects, IEEE Transactions on Education 38(1): 33-39.

Aguirre, L. A. (2000). Introdução à Identificação de Sistemas, Editora UFMG.

Bazaraa, M. S. (1979). Nonlinear Programming, John Wiley and Sons, Inc.

Benchluch, S. M. e Chow, J. H. (1993). A trajectory sensitivity method for the identification of nonlinear excitation system models, IEEE Transaction on Energy Conversion 8(2): 159-164.

Bortoni, E. C. e Jardini, J. A. (2002). Identification of synchronous machine parameters using load rejection test data, IEEE Transactions on Energy Conversion 17(2): 242-247.

Burth, M., Verghese, G. C. e Velez, R. M. (1999). Subset selection for improved parameter estimation in on-line identification of a synchronous generator, IEEE Transaction on Power and Systems 14(1): 218-225.

Cari, E. P. T. (2005). Estimação dos parâmetros da máquina síncrona e seu sistema de excitação, Dissertação de mestrado, Universidade de São Paulo, Escola de engenharia de São Carlos, http://www.teses.usp.br/teses/disponiveis/18/18133/tde-02102005$204356 /$.

Cari, E. P. T., Alberto, L. F. C. e Bretas, N. G. (2006a). A methodology for parameter estimation of synchronous generators based on trajectory sensitivity 
and synchronization technique, IEEE Power Engineering Society General Meeting .

Cari, E. P. T., Alberto, L. F. C. e Bretas, N. G. (2006b). Metodologia para a estimação de parâmetros baseada na técnica de sensibilidade de trajetória e conceitos de sincronização, XVI Congresso Brasileiro de Automática .

Cari, E. P. T., Alberto, L. F. C. e Bretas, N. G. (2008a). A new methodology for parameter estimation of synchronous generator from disturbance measurements, IEEE Power Energy Society General Meeting, Pittsburgh, Pennsylvania, USA .

Cari, E. P. T., Alberto, L. F. C. e Bretas, N. G. (2008b). A trajectory sensitivity based framework to estimate parameters of synchronous generators from disturbance data, Submetido no IEEE Transaction on Energy Conversion .

Cari, E. P. T., Alberto, L. F. C. e Bretas, N. G. (2009a). Nonlinear parameter estimation of synchronous generator using trajectory sensitivity analysis and disturbance measurements, XI Symposium Of Specialists In Electric Operational And Expansion Planning .

Cari, E. P. T., Alberto, L. F. C. e Bretas, N. G. (2009b). A novel methodology for power angle estimation of synchronous generator based on trajectory sensitivity analysis, Submetido no IEEE Power Engineering Society General Meeting, Calgary, Alberta, Canada .

Cari, E. P. T., Alberto, L. F. C., Martins, A. C. P. e Bretas, N. (2005). Methodology for the parameter estimation of synchronous generator and excitation system, IEEE St. Petersburg PowerTech Proceeding .

Cari, E. P. T., Theodoro, E. A. R., Bretas, N. G. e Alberto, L. (2008). Sincronização mestre-escravo aplicada à estimação de sistemas não-lineares, XVII Congresso Brasileiro de Automática, CBA. 
Cari, E. P. T., Theodoro, E. A. R., Mijolaro, A. P., Bretas, N. G. e Alberto, L. F. C. (2009). Trajectory sensitivity method and Master-Slave synchronization to estimate parameters of nonlinear systems, Mathematical Problems in Engineering .

Chen, Y., Zhang, C., Hu, Z. e Wang, X. (2000). A new approach to real time measurement of power angles of generators at different locations for stability control, IEEE Power Engineering Society Winter Meeting 2: 1237-1242.

Chow, J. H., Glinkowski, M., Murphy, R. J., Cease, T. W. e Kosaka, N. (1999). Generator and exciter parameter estimation of fort patrick henry hydro unit 1, IEEE Transactions on Energy Conversion 14: 923-929.

Chua, L. O., Wu, C. W., Huang, A. e G., Z. (1993a). A universal circuit for studying and generating chaos - part i: Routes to chaos, IEEE Transactions on Circuits and Systems 40(10): 732 - 744 .

Chua, L. O., Wu, C. W., Huang, A. e G., Z. (1993b). A universal circuit for studying and generating chaos - part ii: Strange atractors, IEEE Transactionson Circuits and Systems 40(10): 745 - 761 .

Cruz, J. B. (1972). Feedback Systems, McGraw-Hill.

Dandeno, P. L., Kamaker, H. C., C., A., Kamwa, I., Salon, S. J. e Saunder, R. M. (1999). Experience with standstill frequency response (SSFR) testing and analysis of salient pole synchronous machines, IEEE Transactions on Energy Conversion 14(4): 1209-1217.

de Mello, F. P. (1994). Measurement of synchronous machine rotor angle from analysis of zero sequence harmonic components of machine terminal voltage, IEEE Transaction on Power Delivery 9(4): 1170-1177.

Dmitry, K. (2004). Hydro turbine-governor model validation in Pacific Northwest, Transaction on Power on Energy Conversion 19(2): 1144 - 1149. 
Dmitry, K., Carson, W. T. e Willian, A. M. (1999). Model validation for the August 10, 1996 WSCC system outage, IEEE Transactions on Power Systems 14(2): 967-979.

Ferreira, C. M. M., Pinto, J. A. D., Bowler, C. e Barbosa, F. P. M. (2004). Transient stability assessment of an electric power system using trajectory sensitivity analysis, Universities Power Engineering Conference 3: 1091-1095.

Freitas, U. S., Macau, E. E. N. e Grebogi, C. (2005). Using geometric control and chaotic synchronization to estimate an unknown model parameter, Physical Review E 71(047203).

Gameiro, M. e Rodrigues, H. M. (2000). Applications of robust synchronization to communication systems, Applicable Analysis 79(1 - 2): 21-45.

Hannett, L. N. e Feltes, J. W. (1993). Derivation of generator excitation system and turbine governor parameter frond test, Coloquium of CIGRE study committee 38. Florianópolis Brasil .

Hauer, J. F., Beshir, M. J. e Mittelstadt, W. A. (2000). Dynamic performance validation in the western power system, APEx 2000 in Kananaskis Alberta.

Hiramatsu, D., Kakiuchi, M., Uemura, Y., Koyanagi, K., Hirayama, K., Nagano, S., Nagura, R. e Nagasaka, K. (2006). Analytical study on generator load rejection characteristic using advanced equivalent circuit, IEEE Power Engineering Society General Meeting .

Hiskens, I. A. (2001). Nonlinear dynamic model evaluation from disturbance measurements, IEEE Transactions on Power Systems 16(4): 702-710.

Hiskens, I. A. e Koeman, A. (1998). Parameter estimation from power system disturbance measurements, International Conference on Energy Management and Power Delivery 2: 667-672. 
Horning, S., Keyhani, A. e Kamwa, I. (1997). On-line evaluation of a round synchronous machine parameter set estimated from standstill time domain data, IEEE Transactions on Energy Conversion 12(4): 289-296.

Huang, D. (2006). Adaptive-feedback control algorithm, Physical Review E 73(066204): 1-8.

Huang, D. e Guo, R. (2004). Identifying parameter by identical synchronization between different systems, Chaos 14(1): 152-159.

IEEE (1992). Recommended practice for excitation system models for power system stability studies, Technical report, Std 421.5-1992, Power Engineering Society.

IEEE (1995). Guide test procedures for synchronous machines, Technical report, Std 115-1995, Power Engineering Society.

IEEE (2002). Guide for synchronous generator modeling practices and applications in power system stability analysis, Technical report, Std. 1110-2002, Power Engineering Society.

IEEE (2004). Guide for the application of turbine governing systems for hydroelectric generating units, Technical report, IEEE Std 1207TM-2004, Power Engineering Society.

Ines, P. M. e Joaquin, M. (2005). Adaptive approximation method for joint parameter estimation and identical synchronization of chaotic systems, Physical Review E 72(057202).

Jaleeli, N., Bourawi, M. S. e Fish, J. H. (1986). A quasilinearization based algorithm for the identification of transient and subtransient parameters of synchronous machines, IEEE Transaction on Power System 1(3): 46-52.

Jin, Y. Q., Qin, C., Wu, F., Han, J. D., Xu, Q., Yan, D. J. e Ju, P. (2007). The power angle and phase measurement units based wide area measurement 
system and its application, IREP Symposium Bulk Power System Dynamics and Control.

Karaagac, U. e Tor, O. B. (2006). On line parameter identification of a gas turbine generator at Ambarh power plant, IEEE Power Engineering Society General Meeting .

Karrari, M. e Malik, O. P. (2004). Identification of physical parameter of a synchronous generator from online measurements, IEEE Transactions on Energy Conversion 19(2): 407 - 415.

Kundur, P. (1994). Power System Stability and Control, Mc Graw Hill.

LaSalle, J. P. (1976). The Stability of Dynamic Systems, Society for Industrial and Applied Mathematics.

Le, L. X. e Wilson, W. J. (1988). Synchronous machine parameter identification: A time domain approach, IEEE Transaction on Power System 3(2): 241-248.

Ljung, L. (1997). System Identification, Prentice-Hall.

Ma, J., Hogg, B. W., Zhiyuan, N. e Yihan, Y. (1994). On-line decoupled identification of transient and sub-transient generator parameter, IEEE Transaction on Power System 9(4): 1908-1914.

Ong, C.-M. (1998). Dynamic Simulation of Electric Machinery Using Matlab/Simulink, Prentice Hall Inc.

Padiyar, K. R. (1996). Power System Dynamics Stability and Control, John Wiley and Sons (Asia) Pte Ltd.

Parlitz, U. (2006). Adaptive-feedback control algorithm, Physical Review E 73(066204): 1-8.

Pecora, L. M. e Caroll, T. (1990). Synchronization in chaotic systems, Physical Review Letters 64(8): 821-824. 
Rahul, K. (2003). Synchronization-based approach for estimating all model parameters of chaotic systems, Physical Review E 67(027204).

Rodrigues, H. M. (1996). Abstract methods for synchronization and applications, Applicable Analysis an International Journal 62: 263-296.

Sanchez, G. J., Bridenbaugh, C. J., Bowler, C. E. e Edmonds, J. S. (1988). Trajectory sensitivity based identification of synchronous generator and excitation system parameters, IEEE Transaction on Power System 3(4): 1814-1822.

Soliman, S. A. e Al-kandari, A. M. (1996). Optimal parameter estimation of synchronous machines from the digitized sudden short-circuit armature current, IEEE AFRICON 4th 1: 188 - 199.

Sotomayor, J. (1979). Lições de equações diferenciais ordinárias, Rio de Janeiro, IMPA.

Stewart, G. W. (1973). Introduction to Matrix Computation, Academic Press.

Velez, R. M. e Lida, J. (2001). Conditioning analysis of parameter estimation in a synchronous generator, IEEE Electric Machines and Drives Conference pp. $285-291$.

Vermeulen, H. J., Strauss, J. M. e Shikoana, J. M. (2002). Online estimation of synchronous generator parameters using PRBS perturbations, IEEE Transactions on Power System 17(3): 694-700.

Weeber, K. (1997). Determination of dynamic parameter of large hydrogenerators by finite element simulation of three phase sudden short circuit tests, Electric Machines and Drives Conference Record pp. MC/13.1 $-\mathrm{MC} / 13.1$.

Yechiel, C. (2002). A fluid dynamical approach to the control, synchronization and parameter identification of chaotic systems, Proceeding of the American Control Conference 3: 2245-2250. 
Yonathan, B. (1974). Nonlinear Parameter Estimation, Academic-Press Inc.

Yu, W., Chen, G., Cao, J., Lu, J. e Parlitz, U. (2007). Parameter identification of dynamical systems from time series, Physical Review E 75(067201): 1-4.

Zali, S. M., Ariffin, A. E., Mohamed, A. e Hussain, A. (2000). Implementation of synchronous machine parameter derivation in matlab, IEEE TENCON 2000. Proceedings 1: 218-223.

Zhengming, Z., Fungshi, Z., Jide, G. e Longya, X. (1995). A dynamic on-line parameter identification and full-scale system experimental verification for large synchronous machines, Transaction on Power on Energy Conversion 10(1): 392-398. 


\section{Apêndice A}

\section{Publicações Originadas desta Pesquisa}

\section{A.1 Artigos Publicados em Congressos}

- Cari, E. P. T; Alberto, L. F. C, Bretas, N. G. "A Metodology for parameter estimation of synchronous generator base on trajectory sensitivity and synchronization technique" In: 2006 IEEE Power Engineering Society General Meeting, 2006, Montreal, Quebéc, Canada, 2006, 27-30 de Junho de 2006.

- Cari, E. P. T; Alberto, L. F. C, Bretas, N. G. "Metodologia para a estimação de parâmetros baseada na técnica de sensibilidade de trajetória e conceitos de sincronização "In: XVI Congresso brasileiro de Automática, CBA 2006, 3-6 de outubro de 2006, Salvador, Bahia, Brasil.

- Cari, E. P. T; Alberto, L. F. C, Bretas, N. G. "A New Methodology for Parameter Estimation of Synchronous Generator from Disturbance Measurements" IEEE Power Energy Society General Meeting, Pittsburgh, Pennsylvania, USA, July 20-24, 2008.

- Cari, E. P. T; Alberto, L. F. C, Bretas, N. G. "Uma Nova Metodologia Para Estimação de Parâmetros de Geradores Síncronos a Partir de Medidas de Perturbações", Congresso Brasileiro de Automática XVII CBA, 14-17 de 
Setembro de 2008.

- Cari, E. P. T; Alberto, L. F. C, Bretas, N. G. "Sincronização Mestre-Escravo Aplicada à estimação de Sistemas Não-Lineares", Congresso Brasileiro de Automática XVII CBA, 14-17 de Setembro de 2008.

- Cari, E. P. T; Alberto, L. F. C, Bretas, N. G. "Nonlinear Parameter Estimation of Synchronous Generator Using Trajectory Sensitivity Analysis and Disturbance Measurements", XI Symposium Of Specialists In Electric Operational And Expansion Planning, Belen, Brasil 17-20 Mar 2009.

\section{A.2 Artigos Submetidos em Revistas}

- Cari, E. P. T; Alberto, L. F. C, Bretas, N. G. "A Trajectory Sensitivity Based Framework to Estimate Parameters of Synchronous Generators from Disturbance Data" IEEE Transaction on Energy Conversion. Artigo submido em Novembro de 2008.

- Cari, E. P. T; Edson A. R. T.; Ana P. M., Alberto, L. F. C.; Bretas, N. G. "Trajectory Sensitivity Method and Master-Slave Synchronization to Estimate Parameters of Nonlinear Systems "Mathematical Problems in Engineering. Artigo submetido em Fevereiro de 2009.

\section{A.3 Artigos Submetidos em Congressos}

- Cari, E. P. T; Alberto, L. F. C, Bretas, N. G. "A Novel Methodology for Power Angle Estimation of Synchronous Generator Based on Trajectory Sensitivity Analysis" IEEE Power Energy Society General Meeting, Calgary, Alberta, Canada, July 26-30, 2009. Artigo submetido em Dezembro de 2009 


\section{Apêndice B}

\section{Equações do Gerador com a}

\section{Abordagem de Minimização.}

As para solução do sistema auxiliar e das equações de sensibilidade da Seção 5.5 .4 (B) são apresentadas neste apêndice.

\section{B.1 Sistema Auxiliar}

sistema auxiliar é representado pelas equações não-lineares (5.49)-(5.53). A solução destas equações é realizado em forma iterativa pelo método de Newton. Para a iteração $k$, obtém-se:

$$
V_{v}^{k+1}=V_{v}^{k}-\left.\left(\frac{\partial W 1}{\partial V_{v}}\right)^{-1} W 1\right|_{k}
$$

Sendo

$$
V_{v}=\left(E_{q(n+1)}^{\prime}, E_{d(n+1)}^{\prime}, \beta_{(n+1)}, \lambda_{L 1(n+1)}, \lambda_{L 2(n+1)}\right)^{T}
$$

$W 1=\left(\frac{\partial L}{\partial E_{q(n+1)}^{\prime}}, \frac{\partial L}{\partial E_{d(n+1)}^{\prime}}, \frac{\partial L}{\partial \beta_{(n+1)}}, \frac{\partial L}{\partial \lambda_{L 1(n+1)}}, \frac{\partial L}{\partial \lambda_{L 2(n+1)}}\right)^{T}$ (obtida de (5.49)-(5.53)).

A matrix Jacobiana $\frac{\partial W 1}{\partial V_{v}}$ pode ser encontrada derivando-se parcialmente $W 1$ em relação a $V_{v}$.

Derivando-se $W 1$ em relação a $V_{v(1)}=E_{q(n+1)}^{\prime}$ tem-se: 


$$
\begin{aligned}
& \frac{\partial W 1_{(1)}}{\partial V_{v(1)}}=\frac{4}{x_{d}^{\prime}}\left[\frac{\partial g_{(n+1)}}{\partial E_{q(n+1)}^{\prime}} I_{d(n+1)}+g_{(n+1)} \frac{\partial I_{d(n+1)}}{\partial E_{q(n+1)}^{\prime}}\right], \\
& \frac{\partial I_{d(n+1)}}{\partial E_{q(n+1)}^{\prime}}=\frac{-1}{x_{d}^{\prime}} ; \frac{\partial I_{q(n+1)}}{\partial E_{q(n+1)}^{\prime}}=0 ; \frac{\partial g_{(n+1)}}{\partial E_{q(n+1)}^{\prime}}=\frac{2 I_{d(n+1)}}{x_{d}^{\prime}}, \\
& \frac{\partial W 1_{(1)}}{\partial V_{v(1)}}=\frac{4}{x_{d}^{\prime}}\left[\frac{2 I_{d(n+1)}^{2}}{x_{d}^{\prime}}-\frac{g_{(n+1)}}{x_{d}^{\prime}}\right] \text {. } \\
& \frac{\partial W 1_{(2)}}{\partial V_{v(1)}}=\frac{-4}{x_{q}^{\prime}}\left[\frac{\partial g_{(n+1)}}{\partial E_{q(n+1)}^{\prime}} I_{q(n+1)}+g_{(n+1)} \frac{\partial I_{q(n+1)}}{\partial E_{q(n+1)}^{\prime}}\right] \\
& \frac{\partial W 1_{(2)}}{\partial V_{v(1)}}=\frac{-8 I_{d(n+1)} I_{q(n+1)}}{x_{d}^{\prime} x_{q}^{\prime}} \\
& \frac{\partial W 1_{(3)}}{\partial V_{v(1)}}=4 V_{T(n+1)}\left[\frac{\partial g_{(n+1)}}{\partial E_{q(n+1)}^{\prime}}\left(\frac{I_{d(n+1)} \operatorname{sen}\left(\beta_{(n+1)}\right)}{x_{d}^{\prime}}-\frac{I_{q(n+1)} \cos \left(\beta_{(n+1)}\right)}{x_{q}^{\prime}}\right)+\right. \\
& \left.g_{(n+1)}\left(\frac{\operatorname{sen}\left(\beta_{(n+1)}\right)}{x_{d}^{\prime}} \frac{\partial I_{d(n+1)}}{\partial E_{q(n+1)}^{\prime}}-\frac{\cos \left(\beta_{(n+1)}\right)}{x_{q}^{\prime}} \frac{\partial I_{q(n+1)}}{\partial E_{q(n+1)}^{\prime}}\right)\right], \\
& \frac{\partial W 1_{(3)}}{\partial V_{v(1)}}=\frac{4 V_{T(n+1)}}{x_{d}^{\prime}}\left[2 I_{d(n+1)}\left(\frac{I_{d(n+1)} \operatorname{sen}\left(\beta_{(n+1)}\right)}{x_{d}^{\prime}}-\frac{I_{q(n+1)} \cos \left(\beta_{(n+1)}\right)}{x_{q}^{\prime}}\right)-\right. \\
& \left.\frac{g_{(n+1)} \operatorname{sen}\left(\beta_{(n+1)}\right)}{x_{d}^{\prime}}\right] \text {, } \\
& \frac{\partial W 1_{(4)}}{\partial V_{v(1)}}=1-\frac{\Delta t}{2 T_{d o}^{\prime}}\left[-1+\left(x_{d}-x_{d}^{\prime}\right) \frac{\partial I_{d(n+1)}}{\partial E_{q(n+1)}^{\prime}}\right]=1+\frac{\Delta t x_{d}}{2 T_{d o}^{\prime} x_{d}^{\prime}} . \\
& \frac{\partial W 1_{(5)}}{\partial V_{v(1)}}=\frac{\Delta t}{2 T_{q o}^{\prime}}\left(x_{q}-x_{q}^{\prime}\right) \frac{\partial I_{q(n+1)}}{\partial E_{q(n+1)}^{\prime}}, \\
& \frac{\partial W 1_{(5)}}{\partial V_{v(1)}}=0 \text {. }
\end{aligned}
$$

Derivando-se $W 1$ em relação a $V_{v(2)}=E_{d(n+1)}^{\prime}$ tem-se:

$$
\begin{aligned}
\frac{\partial W 1_{(1)}}{\partial V_{v(2)}} & =\frac{4}{x_{d}^{\prime}}\left[\frac{\partial g_{(n+1)}}{\partial E_{d(n+1)}^{\prime}} I_{d(n+1)}+g_{(n+1)} \frac{\partial I_{d(n+1)}}{\partial E_{d(n+1)}^{\prime}}\right] \\
\frac{\partial I_{d(n+1)}}{\partial E_{d(n+1)}^{\prime}} & =0 ; \frac{\partial I_{q(n+1)}}{\partial E_{d(n+1)}^{\prime}}=\frac{1}{x_{q}^{\prime}} ; \frac{\partial g_{(n+1)}}{\partial E_{d(n+1)}^{\prime}}=\frac{-2 I_{q(n+1)}}{x_{q}^{\prime}} \\
\frac{\partial W 1_{(1)}}{\partial V_{v(2)}} & =\frac{-8 I_{d(n+1)} I_{q(n+1)}}{x_{d}^{\prime} x_{q}^{\prime}} \\
\frac{\partial W 1_{(2)}}{\partial V_{v(2)}} & =\frac{-4}{x_{q}^{\prime}}\left[\frac{\partial g_{(n+1)}}{\partial E_{d(n+1)}^{\prime}} I_{q(n+1)}+g_{(n+1)} \frac{\partial I_{q(n+1)}}{\partial E_{d(n+1)}^{\prime}}\right] \\
\frac{\partial W 1_{(2)}}{\partial V_{v(2)}} & =\frac{8 I_{q(n+1)}^{2}-4 g_{(n+1)}}{x_{q}^{\prime 2}}
\end{aligned}
$$




$$
\begin{aligned}
\frac{\partial W 1_{(3)}}{\partial V_{v(2)}}= & 4 V_{T(n+1)}\left[\frac{\partial g_{(n+1)}}{\partial E_{d(n+1)}^{\prime}}\left(\frac{I_{d(n+1)} \operatorname{sen}\left(\beta_{(n+1)}\right)}{x_{d}^{\prime}}-\frac{I_{q(n+1)} \cos \left(\beta_{(n+1)}\right)}{x_{q}^{\prime}}\right)+\right. \\
& g_{(n+1)}\left(\frac{\operatorname{sen}\left(\beta_{(n+1)}\right)}{x_{d}^{\prime}} \frac{\partial I_{d(n+1)}}{\partial E_{d(n+1)}^{\prime}}-\frac{\cos \left(\beta_{(n+1)}\right)}{x^{\prime} q} \frac{\partial I_{q(n+1)}}{\left.\left.\partial E_{d(n+1)}^{\prime}\right)\right],}\right. \\
\frac{\partial W 1_{(3)}}{\partial V_{v(2)}}= & \frac{-4 V_{T(n+1)}}{x_{q}^{\prime}}\left[\frac{2 I_{d(n+1)} I_{q(n+1)} \operatorname{sen}\left(\beta_{(n+1)}\right)}{x_{d}^{\prime}}-\frac{2 I_{q(n+1)}^{2} \cos \left(\beta_{(n+1)}\right)}{x_{q}^{\prime}}+\right. \\
\left.\frac{g_{(n+1)} \cos \left(\beta_{(n+1)}\right)}{x_{q}^{\prime}}\right] & 0 \\
\frac{\partial W 1_{(4)}}{\partial V_{v(2)}}= & 1+\frac{\Delta t}{2 T_{q o}^{\prime}}\left[1+\left(x_{q}-x_{q}^{\prime}\right) \frac{\partial I_{q(n+1)}}{\partial E_{d(n+1)}^{\prime}}\right]=1+\frac{\Delta t x_{q}}{2 T_{q o}^{\prime} x_{q}^{\prime}} .
\end{aligned}
$$

Derivando-se $W 1$ em relação a $V_{v(3)}=\beta_{(n+1)}$ tem-se:

$$
\begin{aligned}
& \frac{\partial W 1_{(1)}}{\partial V_{v(3)}}=\frac{4}{x_{d}^{\prime}}\left[\frac{\partial g_{(n+1)}}{\partial \beta_{(n+1)}} I_{d(n+1)}+g_{(n+1)} \frac{\partial I_{d(n+1)}}{\partial \beta_{(n+1)}}\right] \\
& \frac{\partial I_{d(n+1)}}{\partial \beta_{(n+1)}}=\frac{-V_{T(n+1)} \operatorname{sen}\left(\beta_{(n+1)}\right)}{x_{d}^{\prime}} ; \frac{\partial I_{q(n+1)}}{\partial \beta_{(n+1)}}=\frac{V_{T(n+1)} \cos \left(\beta_{(n+1)}\right)}{x_{q}^{\prime}}, \\
& \frac{\partial g_{(n+1)}}{\partial \beta_{(n+1)}}=\frac{2 I_{d(n+1)} V_{T(n+1)} \operatorname{sen}\left(\beta_{(n+1)}\right)}{x_{d}^{\prime}}-\frac{2 I_{q(n+1)} V_{T(n+1)} \cos \left(\beta_{(n+1)}\right)}{x_{q}^{\prime}}, \\
& \frac{\partial W 1_{(1)}}{\partial V_{v(3)}}=\frac{4 V_{T(n+1)}}{x_{d}^{\prime}}\left[2 I_{d(n+1)}\left(\frac{I_{d(n+1)} \operatorname{sen}\left(\beta_{(n+1)}\right)}{x_{d}^{\prime}}-\frac{I_{q(n+1)} \cos \left(\beta_{(n+1)}\right)}{x_{q}^{\prime}}\right)-\right. \\
& \left.\frac{g_{(n+1)} \operatorname{sen}\left(\beta_{(n+1)}\right)}{x_{d}^{\prime}}\right] \\
& \frac{\partial W 1_{(2)}}{\partial V_{v(3)}}=\frac{-4 V_{T(n+1)}}{x_{q}^{\prime}}\left[\frac{2 I_{d(n+1)} I_{q(n+1)} \operatorname{sen}\left(\beta_{(n+1)}\right)}{x_{d}^{\prime}}-\frac{2 I_{q(n+1)}^{2} \cos \left(\beta_{(n+1)}\right)}{x_{q}^{\prime}}+\right. \\
& \left.\frac{g_{(n+1)} \cos \left(\beta_{(n+1)}\right)}{x_{q}^{\prime}}\right] \\
& \frac{\partial W 1_{(3)}}{\partial V_{v(3)}}=4 V_{T(n+1)}\left\{2 V_{T(n+1)}\left(\frac{I_{d(n+1)} \operatorname{sen}\left(\beta_{(n+1)}\right)}{x_{d}^{\prime}}-\frac{I_{q(n+1)} \cos \left(\beta_{(n+1)}\right)}{x_{q}^{\prime}}\right)^{2}+\right. \\
& g_{(n+1)}\left[\frac{1}{x_{d}^{\prime}}\left(\frac{-V_{T(n+1)} \operatorname{sen}^{2}\left(\beta_{(n+1)}\right)}{x_{d}^{\prime}}+I_{d(n+1)} \cos \left(\beta_{(n+1)}\right)\right)\right. \\
& \left.\left.-\frac{1}{x_{q}^{\prime}}\left(\frac{V_{T(n+1)} \cos ^{2}\left(\beta_{(n+1)}\right)}{x_{q}^{\prime}}-I_{q(n+1)} \operatorname{sen}\left(\beta_{(n+1)}\right)\right)\right]\right\}+ \\
& \frac{\mu_{1} \Delta t\left(x_{d}-x_{d}^{\prime}\right) V_{T(n+1)} \cos \left(\beta_{(n+1)}\right)}{2 T_{d o}^{\prime} x_{d}^{\prime}}-\frac{\mu_{2} \Delta t\left(x_{q}-x_{q}^{\prime}\right) V_{T(n+1)} \operatorname{sen}\left(\beta_{(n+1)}\right)}{2 T_{q o}^{\prime} x_{q}^{\prime}}
\end{aligned}
$$




$$
\begin{aligned}
\frac{\partial W 1_{(4)}}{\partial V_{v(3)}} & =\frac{\Delta t\left(x_{d}-x_{d}^{\prime}\right) V_{T(n+1)} \operatorname{sen}\left(\beta_{(n+1)}\right)}{2 T_{d o}^{\prime} x_{d}^{\prime}} \\
\frac{\partial W 1_{(5)}}{\partial V_{v(3)}} & =\frac{\Delta t\left(x_{q}-x_{q}^{\prime}\right) V_{T(n+1)} \cos \left(\beta_{(n+1)}\right)}{2 T_{q o}^{\prime} x_{q}^{\prime}}
\end{aligned}
$$

Derivando-se $W 1$ em relação a $V_{v(4)}=\mu_{1(n+1)}$ tem-se:

$$
\begin{aligned}
& \frac{\partial W 1_{(1)}}{\partial V_{v(4)}}=1+\frac{\Delta t x_{d}}{2 T_{d o}^{\prime} x_{d}^{\prime}}, \\
& \frac{\partial W 1_{(2)}}{\partial V_{v(4)}}=0, \\
& \frac{\partial W 1_{(3)}}{\partial V_{v(4)}}=\frac{\Delta t\left(x_{d}-x_{d}^{\prime}\right) V_{T(n+1)} \operatorname{sen}\left(\beta_{(n+1)}\right)}{2 T_{d o}^{\prime} x_{d}^{\prime}}, \\
& \frac{\partial W 1_{(4)}}{\partial V_{v(4)}}=0, \\
& \frac{\partial W 1_{(5)}}{\partial V_{v(4)}}=0,
\end{aligned}
$$

Derivando-se $W 1$ em relação a $V_{v(5)}=\mu_{2(n+1)}$ tem-se:

$$
\begin{aligned}
\frac{\partial W 1_{(1)}}{\partial V_{v(5)}} & =0, \\
\frac{\partial W 1_{(2)}}{\partial V_{v(5)}} & =1+\frac{\Delta t x_{q}}{2 T_{q o}^{\prime} x_{q}^{\prime}}, \\
\frac{\partial W 1_{(3)}}{\partial V_{v(5)}} & =\frac{\Delta t\left(x_{q}-x_{q}^{\prime}\right) V_{T(n+1)} \cos \left(\beta_{(n+1)}\right)}{2 T_{q o}^{\prime} x_{q}^{\prime}}, \\
\frac{\partial W 1_{(4)}}{\partial V_{v(5)}} & =0, \\
\frac{\partial W 1_{(5)}}{\partial V_{v(5)}} & =0
\end{aligned}
$$

Portanto a matriz Jacobiana é:

$$
\frac{\partial W 1}{\partial V_{v}}=\left(\begin{array}{cccc}
\frac{\partial W 1_{(1)}}{\partial V_{v(1)}} & \frac{\partial W 1_{(1)}}{\partial V_{v(2)}} & \cdots & \frac{\partial W 1_{(1)}}{\partial V_{v(5)}} \\
\frac{\partial W 1_{(2)}}{\partial V_{v(1)}} & \frac{\partial W 1_{(2)}}{\partial V_{v(2)}} & \cdots & \frac{\partial W 1_{(2)}}{\partial V_{v(5)}} \\
\vdots & \vdots & \ddots & \vdots \\
\frac{\partial W 1_{(5)}}{\partial V_{v(1)}} & \frac{\partial W 1_{(5)}}{\partial V_{v(2)}} & \cdots & \frac{\partial W 1_{(5)}}{\partial V_{v(5)}}
\end{array}\right)
$$

A qual é utilizada na equação (B.1) para obter $E_{q(n+1)}^{\prime}, E_{d(n+1)}^{\prime}, \beta_{(n+1)}, \lambda_{L 1(n+1)}$ e $\lambda_{L 2(n+1)}$. Este procedimento é repetido para todo "n" dentro do intervalo de tempo das medidas aferidas. No final $E_{d}^{\prime}(t), E_{d}^{\prime}(t), \beta(t), \lambda_{L 1}(t), \lambda_{L 2}(t)$ serão totalmente determinadas. 


\section{B.2 Equações de Sensibilidade e Funções de Sen- sibilidade de Trajetória}

Esta seção apresenta as equações de sensibilidade para a metodologia de sensibilidade de trajetória utilizando a abordagem de minimização (vide seção 5.5.4(B)).

A partir das equações não-lineares do sistema auxiliar $W 1$ obtida de (5.49)(5.53) e o vetor de parâmetros $p=\left(x_{d}, x_{d}^{\prime}, T_{d o}^{\prime}, x_{q}, x_{q}^{\prime}, T_{q o}^{\prime}, E_{q o}^{\prime}, E_{d o}^{\prime}\right)^{T}$, as equações de sensibilidade são obtidas derivando-se $W 1$ em relação a cada parâmetro e igualando a zero o conjunto de equações não-lineares resultante, como é mostrado a seguir.

Para o parâmetro $x_{d}$, denota-se as seguintes variáveis:

$$
U=\left(\begin{array}{c}
\frac{\partial W 1_{(1)}}{\partial x_{d}} \\
\frac{\partial W 1_{(2)}}{\partial x_{d}} \\
\vdots \\
\frac{\partial W 1_{(5)}}{\partial x_{d}}
\end{array}\right)=0 ; \quad Z_{z}=\left(\begin{array}{c}
\lambda_{E_{q(n+1)}^{\prime}}^{x_{d}} \\
\lambda_{E_{d(n+1)}^{\prime}}^{x_{d}} \\
\lambda_{\beta_{(n+1)}}^{x_{d}} \\
\lambda_{\mu_{1(n+1)}}^{x_{d}} \\
\lambda_{\mu_{2(n+1)}}^{x_{d}}
\end{array}\right)
$$

Sendo $\lambda_{f}^{p_{i}}=\frac{\partial f}{p_{i}}, U$ o vetor de equações não lineares que compõem as equações de sensibilidade e $Z_{z}$ o vetor que contém as funções de sensibilidade, as quais podem ser encontradas utilizando o método de Newton.

Utilizando o método de Newton, na iteração k, tem-se:

$$
Z_{z}^{k+1}=Z_{z}^{k}-\left.\left(\frac{\partial U}{\partial Z_{z}}\right)^{-1} U\right|_{k}
$$

Durante o processo de calculo utiliza-se o fato que a matriz Jacobiana das funções de sensibilidade $\left(\frac{\partial U}{\partial Z_{z}}\right)$ é a mesma que a matriz Jacobiana da solução das equações do sistema auxiliar $\left(\frac{\partial W 1}{\partial V_{v}}\right)$.

As equações de sensibilidade das variáveis de estado para o parâmetro $x_{d}$ são as seguintes: 


$$
\begin{aligned}
& \frac{\partial U_{(1)}}{x_{d}}=\frac{4}{x_{d}^{\prime}}\left[\frac{\partial g_{(n+1)}}{x_{d}} I_{d(n+1)}+g_{(n+1)} \frac{\partial I_{d(n+1)}}{x_{d}}\right]+ \\
& \lambda_{u_{1(n+1)}}^{x_{d}}\left[1+\frac{\Delta t x_{d}}{2 T_{d o}^{\prime} x_{d}^{\prime}}\right]+\frac{\mu_{1(n+1)} \Delta t}{2 T_{d o}^{\prime} x_{d}^{\prime}} \\
& \frac{\partial U_{(2)}}{x_{d}}=\frac{-4}{x_{q}^{\prime}}\left[\frac{\partial g_{(n+1)}}{x_{d}} I_{q(n+1)}+g_{(n+1)} \frac{\partial I_{q(n+1)}}{x_{d}}\right]+, \\
& \lambda_{u_{2(n+1)}}^{x_{d}}\left[1+\frac{\Delta t x_{q}}{2 T_{q o}^{\prime} x_{q}^{\prime}}\right] \\
& \frac{\partial U_{(3)}}{x_{d}}=4 V_{T(n+1)}\left\{\frac{\partial g_{(n+1)}}{x_{d}}\left[\frac{I_{d(n+1)} \operatorname{sen}\left(\beta_{(n+1)}\right)}{x_{d}^{\prime}}-\frac{I_{q(n+1)} \cos \left(\beta_{(n+1)}\right)}{x_{q}^{\prime}}\right]+\right. \\
& g_{(n+1)}\left[\frac{1}{x_{d}^{\prime}}\left(\frac{\partial I_{d(n+1)}}{\partial x_{d}^{\prime}} \operatorname{sen}\left(\beta_{(n+1)}\right)+I_{d(n+1)} \cos \left(\beta_{(n+1)}\right) \lambda_{\beta_{n+1}}^{x_{d}}\right)\right. \\
& \left.\left.-\frac{1}{x_{q}^{\prime}}\left(\frac{\partial I_{q(n+1)}}{\partial x_{d}^{\prime}} \cos \left(\beta_{(n+1)}\right)-I_{q(n+1)} \operatorname{sen}\left(\beta_{(n+1)}\right) \lambda_{\beta_{n+1}}^{x_{d}}\right)\right]\right\}+ \\
& \frac{\Delta t V_{T(n+1)}}{2 T_{d o}^{\prime} x_{d}^{\prime}}\left[\lambda_{\mu_{1(n+1)}}^{x_{d}}\left(x_{d}-x_{d}^{\prime}\right) \operatorname{sen}\left(\beta_{n+1}\right)+\mu_{1(n+1)} \operatorname{sen}\left(\beta_{(n+1)}\right)+\right. \\
& \left.\mu_{1(n+1)}\left(x_{d}-x_{d}^{\prime}\right) \cos \left(\beta_{(n+1)}\right) \lambda_{\beta_{n+1}}^{x_{d}}\right]+\frac{\Delta t V_{T(n+1)}\left(x_{q}-x_{q}^{\prime}\right)}{2 T_{q o}^{\prime} x_{q}^{\prime}}\left[\lambda_{\mu_{2(n+1)}}^{x_{d}} \cos \left(\beta_{n+1}\right)\right. \\
& \left.-\mu_{2(n+1)} \operatorname{sen}\left(\beta_{(n+1)}\right) \lambda_{\beta_{(n+1)}}^{x_{d}}\right] \\
& \frac{\partial U_{(4)}}{x_{d}}=\lambda_{E_{q(n+1)}^{\prime}}^{x_{d}}-\frac{\Delta t}{2 T_{d o}^{\prime}}\left[-\lambda_{E_{q(n+1)}^{\prime}}^{x_{d}}+I_{d(n+1)}+\left(x_{d}-x_{d}^{\prime}\right) \frac{\partial I_{d(n+1)}}{\partial x_{d}}\right]-\lambda_{E_{q(n)}^{\prime}}^{x_{d}} \\
& -\frac{\Delta t}{2 T_{d o}^{\prime}}\left[-\lambda_{E_{q(n)}^{\prime}}^{x_{d}}+I_{d(n)}+\left(x_{d}-x_{d}^{\prime}\right) \frac{\partial I_{d(n)}}{\partial x_{d}}\right] \\
& \frac{\partial U_{(5)}}{x_{d}}=\lambda_{E_{d(n+1)}^{\prime}}^{x_{d}}+\frac{\Delta t}{2 T_{q o}^{\prime}}\left[\lambda_{E_{d(n+1)}^{\prime}}^{x_{d}}+\left(x_{q}-x_{q}^{\prime}\right) \frac{\partial I_{q(n+1)}}{\partial x_{d}}\right]-\lambda_{E_{d(n)}^{\prime}}^{x_{d}}+ \\
& \frac{\Delta t}{2 T_{q o}^{\prime}}\left[\lambda_{E_{d(n)}^{\prime}}^{x_{d}}+\left(x_{q}-x_{q}^{\prime}\right) \frac{\partial I_{q(n)}}{\partial x_{d}}\right]
\end{aligned}
$$

Sendo:

$$
\begin{aligned}
& \frac{\partial I_{d(n+1)}}{\partial x_{d}}=\frac{-V_{T(n+1)} \operatorname{sen}\left(\beta_{(n+1)}\right) \lambda_{\beta_{n+1}}^{x_{d}}-\lambda_{E_{q(n+1)}^{x_{d}}}^{x_{d}^{\prime}}}{\frac{\partial I_{q(n+1)}}{\partial x_{d}}}=\frac{V_{T(n+1)} \cos \left(\beta_{(n+1)}\right) \lambda_{\beta_{n+1}}^{x_{d}}+\lambda_{E_{d(n+1)}^{x_{d}}}}{x_{q}^{\prime}} \\
& \frac{g_{(n+1)}}{\partial x_{d}}=-2 I_{d(n+1) \frac{\partial I_{d(n+1)}}{\partial x_{d}}-2 I_{q(n+1)} \frac{\partial I_{q(n+1)}}{\partial x_{d}}} \\
& \frac{\partial I_{d(n)}}{\partial x_{d}}=\frac{-V_{T(n)} \operatorname{sen}\left(\beta_{(n)}\right) \lambda_{\beta_{n}}^{x_{d}}-\lambda_{E_{q(n)}^{\prime}}^{x_{d}}}{x_{d}^{\prime}}
\end{aligned}
$$




$$
\begin{aligned}
& \frac{\partial I_{q(n)}}{\partial x_{d}}=\frac{V_{T(n)} \cos \left(\beta_{(n)}\right) \lambda_{\beta_{n}}^{x_{d}}+\lambda_{E_{d(n)}^{\prime}}^{x_{d}}}{x_{q}^{\prime}}, \\
& \frac{g_{(n)}}{\partial x_{d}}=-2 I_{d(n)} \frac{\partial I_{d(n)}}{\partial x_{d}}-2 I_{q(n)} \frac{\partial I_{q(n)}}{\partial x_{d}} .
\end{aligned}
$$

Portanto a matriz Jacobiana é:

$$
\frac{\partial U}{\partial Z_{z}}=\left(\begin{array}{cccc}
\frac{\partial U_{(1)}}{\partial Z_{z(1)}} & \frac{\partial U_{(1)}}{\partial Z_{z(2)}} & \cdots & \frac{\partial U_{(1)}}{\partial Z_{z(5)}} \\
\frac{\partial U_{(2)}}{\partial Z_{z(1)}} & \frac{\partial U_{(2)}}{\partial Z_{z(2)}} & \cdots & \frac{\partial U_{(2)}}{\partial Z_{z(5)}} \\
\vdots & \vdots & \ddots & \vdots \\
\frac{\partial U_{(5)}}{\partial Z_{z(1)}} & \frac{\partial U_{(5)}}{\partial Z_{z(2)}} & \cdots & \frac{\partial U_{(5)}}{\partial Z_{z(5)}}
\end{array}\right)
$$

A qual é utilizada na equação (B.27) para obter $\lambda_{E_{q(n+1)}^{\prime}}^{x_{d}}, \lambda_{E_{d(n+1)}^{\prime}}^{x_{d}}, \lambda_{\beta_{(n+1)}}^{x_{d}}$. Este procedimento é repetido para todo "n" dentro do intervalo de tempo das medidas aferidas. No final $\lambda_{E_{q(t)}^{\prime}}^{x_{d}}, \lambda_{E_{d(t)}^{\prime}}^{x_{d}}, \lambda_{\beta_{(t)}}^{x_{d}}$ serão totalmente determinadas.

As funções de sensibilidade de trajetória das saídas podem ser facilmente determinadas derivando-se (5.22) e (5.23) em relação a $x_{d}$ e substituindo as variáveis anteriormente encontradas.

$$
\begin{aligned}
& \lambda_{P_{e}}^{x_{d}}=\lambda_{E_{d}^{\prime}}^{x_{d}} I_{d}+E_{d}^{\prime} \frac{\partial I_{d}}{\partial x_{d}}+\lambda_{E_{q}^{\prime}}^{x_{d}} I_{q}+E_{q}^{\prime} \frac{\partial I_{q}}{\partial x_{d}}+\left(x_{d}^{\prime}-x_{q}^{\prime}\right)\left(\frac{\partial I_{d}}{\partial x_{d}} I_{q}+I_{d} \frac{\partial I_{q}}{\partial x_{d}}\right) \\
& \lambda_{Q_{e}}^{x_{d}}=\lambda_{E_{d}^{\prime}}^{x_{d}} I_{q}+E_{d}^{\prime} \frac{\partial I_{q}}{\partial x_{d}}-\lambda_{E_{q}^{\prime}}^{x_{d}} I_{d}-E_{q}^{\prime} \frac{\partial I_{d}}{\partial x_{d}}-2 x_{q}^{\prime} I_{q} \frac{\partial I q}{\partial x_{d}}-2 x_{d}^{\prime} I_{d} \frac{\partial I d}{\partial x_{d}}
\end{aligned}
$$

As funções de sensibilidade para os outros parâmetros podem ser determinadas seguindo o mesmo procedimento,. 
